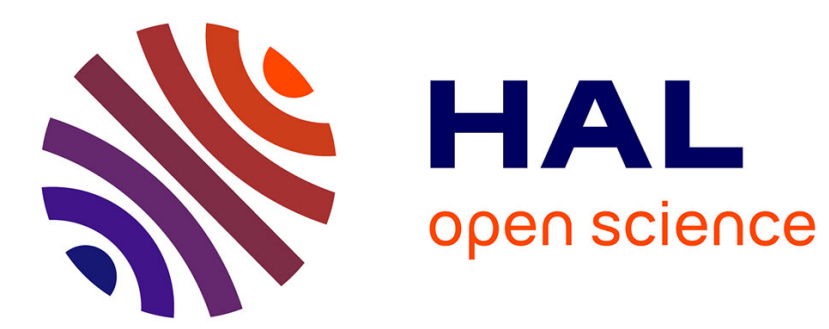

\title{
BUBBLING ABOVE THE THRESHOLD OF THE SCALAR CURVATURE IN DIMENSIONS FOUR AND FIVE
}

Bruno Premoselli, Pierre-Damien Thizy

\section{- To cite this version:}

Bruno Premoselli, Pierre-Damien Thizy. BUBBLING ABOVE THE THRESHOLD OF THE SCALAR CURVATURE IN DIMENSIONS FOUR AND FIVE. 2018. hal-01805092

\section{HAL Id: hal-01805092 \\ https://hal.science/hal-01805092}

Preprint submitted on 1 Jun 2018

HAL is a multi-disciplinary open access archive for the deposit and dissemination of scientific research documents, whether they are published or not. The documents may come from teaching and research institutions in France or abroad, or from public or private research centers.
L'archive ouverte pluridisciplinaire HAL, est destinée au dépôt et à la diffusion de documents scientifiques de niveau recherche, publiés ou non, émanant des établissements d'enseignement et de recherche français ou étrangers, des laboratoires publics ou privés. 


\title{
BUBBLING ABOVE THE THRESHOLD OF THE SCALAR CURVATURE IN DIMENSIONS FOUR AND FIVE.
}

\author{
BRUNO PREMOSELLI AND PIERRE-DAMIEN THIZY
}

\begin{abstract}
On any closed manifold $\left(M^{n}, g\right)$ of dimension $n \in\{4,5\}$ we exhibit new blow-up configurations for perturbations of a purely critical stationary Schrödinger equation. We construct positive solutions which blow-up as the sum of two isolated bubbles, one of which concentrates at a point $\xi$ where the potential $k$ of the equation satisfies

$$
k(\xi)>\frac{n-2}{4(n-1)} S_{g}(\xi),
$$

where $S_{g}$ is the scalar curvature of $\left(M^{n}, g\right)$. The latter condition requires the bubbles to blow-up at different speeds and forces us to work at an elevated precision. We take care of this by performing a construction which combines a priori asymptotic analysis methods with a Lyapounov-Schmidt reduction.
\end{abstract}

\section{INTRODUCTION}

1.1. Statement of the results. Let $(M, g)$ be a smooth closed Riemannian manifold of dimension $n \geq 3$. Let $\triangle_{g}=-\operatorname{div}_{g}(\nabla \cdot)$ be the Laplace-Beltrami operator and let $k$ be a smooth function in $M$ such that $\triangle_{g}+k$ is coercive. We are interested in this paper in the existence of energy-bounded blowing-up families of positive solutions $\left(u_{\varepsilon}\right)_{\varepsilon>0}$ to critical stationary Schrödinger equations of the following type:

$$
\triangle_{g} u_{\varepsilon}+k_{\varepsilon} u_{\varepsilon}=u_{\varepsilon}^{2^{*}-1} \quad \text { in } M,
$$

where $2^{*}=\frac{2 n}{n-2}$ is the critical power for the embedding of $H^{1}(M)$ into Lebesgue spaces and $\left(k_{\varepsilon}\right)_{\varepsilon>0}$ is a smooth perturbation of $k$. We say that a family $\left(u_{\varepsilon}\right)_{\varepsilon>0}$ of solutions of (1.1) has bounded energy if

$$
\limsup _{\varepsilon \rightarrow 0}\left\|u_{\varepsilon}\right\|_{H^{1}(M)}<+\infty .
$$

Since the work of Struwe [26] it is known that if $\left(u_{\varepsilon}\right)_{\varepsilon>0}$ has bounded energy then, up to a subsequence, there exist $k \in \mathbb{N}, k$ sequences $\left(\mu_{1, \varepsilon}\right)_{\varepsilon}, \ldots,\left(\mu_{k, \varepsilon}\right)_{\varepsilon}$ of positive real numbers converging to zero and $k$ sequences $\left(\xi_{1, \varepsilon}\right)_{\varepsilon}, \ldots,\left(\xi_{k, \varepsilon}\right)_{\varepsilon}$ of points of $M$ such that

$$
u_{\varepsilon}=u_{0}+\sum_{i=1}^{k} W_{i, \varepsilon}+o(1) \text { in } H^{1}(M),
$$

The first author is supported by a FNRS grant MIS F.4522.15. 
where the $W_{i, \varepsilon}$ are bubbling profiles given by

$$
W_{i, \varepsilon}=\left(\frac{\mu_{i, \varepsilon}}{\mu_{i, \varepsilon}^{2}+\frac{d_{g}\left(\xi_{i, \varepsilon},\right)^{2}}{n(n-2)}}\right)^{\frac{n-2}{2}},
$$

$d_{g}$ is the geodesic distance and $u_{\varepsilon} \rightarrow u_{0}$ as $\varepsilon \rightarrow 0$. We say that the family $\left(u_{\varepsilon}\right)_{\varepsilon>0}$ of solutions of (1.1) blows-up if

$$
\limsup _{\varepsilon \rightarrow 0}\left\|u_{\varepsilon}\right\|_{C^{0}(M)}=+\infty .
$$

If $\left(u_{\varepsilon}\right)_{\varepsilon>0}$ has bounded energy and blows-up it is easily seen that $k \geq 1$ in 1.2 .

In the last decades, a vast amount of work was poured into understanding when equations (1.1) possess blowing-up families of positive solutions - with and without the energybound assumption. It turns out that the geometric potential $k \equiv c_{n} S_{g}$ plays a threshold role, where we have let $c_{n}=\frac{n-2}{4(n-1)}$ and where $S_{g}$ denotes the scalar curvature of $(M, g)$. It was indeed proven in [6] that when $n \geq 4$ (1.1) has no blowing-up positive solutions whatsoever if $k<c_{n} S_{g}$, and in [5] that (1.1) has no energy-bounded blowingup positive solutions if $k>c_{n} S_{g}$ (unless maybe if $n=6$, see [5]). When $n=3$ the situation is completely different, see [9]. The latter result is based on the generalization of decomposition $(1.2)$ to $C^{0}(M)$ obtained in [7]. On the other side, energy-bounded blowing-up families of positive solutions of (1.1) have been constructed when $k_{\varepsilon}$ is a small perturbation of $c_{n} S_{g}$, see for instance [8, 17, 25. In another direction, if $k_{\varepsilon} \equiv c_{n} S_{g}$ for all $\varepsilon$, equation (1.1) is the Yamabe equation, and its compactness properties exhibit intriguing dimensional phenomena, see [2, 5, 11, 13, 14, 15].

In this article we construct, in dimensions four and five, exotic bubbling configurations for (1.1), where the potential $k$ lies well above the threshold of the scalar curvature at one of the concentration points. In particular, our equations are not perturbations of the Yamabe equation. Let $(M, g)$ be a $n$-dimensional closed Riemannian manifold, $n \in\{4,5\}$, and let $\Psi \in C_{c}^{\infty}\left(\mathbb{R}^{n}\right)$ be a smooth compactly supported function in $B_{0}\left(R_{0}\right) \subset \mathbb{R}^{n}$ for some $R_{0}>1$. Assume that $\Psi>0$ in $B_{0}(1)$ and that $\Psi$ has a non-degenerate global maximum at 0 . Let $\xi_{2,0} \in M$ be fixed. We let $i_{g}$ denote the injectivity radius of $(M, g)$ and for $0<\delta<i_{g} / R_{0}$ we let $h_{\delta}$ be given by

$$
h_{\delta}(x)=\Psi\left(\frac{1}{\delta} \exp _{\xi_{2,0}}^{-1}(x)\right) .
$$

In particular, $h_{\delta}$ is supported in the geodesic ball $B_{g}\left(\xi_{2,0}, R_{0} \delta\right)$, and is allowed to change sign if $\Psi$ changes sign. Remember that $(M, g)$ is said to be of positive Yamabe type if $\triangle_{g}+c_{n} S_{g}$ is a positive operator. Our main result states as follows.

Theorem 1.1. Let $(M, g)$ be a closed Riemannian manifold of dimension $n \in\{4,5\}$ of positive Yamabe type, not conformally diffeomorphic to the standard sphere $\left(\mathbb{S}^{n}, g_{\text {std }}\right)$. Let $\xi_{1,0}$ and $\xi_{2,0}$ be distinct points in $M$ and define $h_{\delta}$ as in (1.4). Let $\delta>0$ small be fixed and let $H$ be any function in the class $\mathcal{C}(\mathcal{H})$ defined in (1.7) below. Then, for any $0<\varepsilon \leq \varepsilon_{0}$ small enough, there exists a positive solution $u_{\varepsilon}$ of:

$$
\triangle_{g} u_{\varepsilon}+\left(c_{n} S_{g}+h_{\delta}+\varepsilon H\right) u_{\varepsilon}=u_{\varepsilon}^{2^{*}-1}
$$


in $M$. This family $\left(u_{\varepsilon}\right)_{0<\varepsilon \leq \varepsilon_{0}}$ blows-up with finite energy at two distincts simple blow-up points as $\varepsilon \rightarrow 0$ and has a zero weak limit.

Note that when $(M, g)$ is of positive Yamabe type and $h_{\delta}$ is given by $(1.4)$ the operator $\triangle_{g}+c_{n} S_{g}+h_{\delta}$ remains positive for small $\delta$. On the other side, the nonnegativity of $\triangle_{g}+c_{n} S_{g}+h_{\delta}$ is a necessary condition to the existence of positive solutions of (1.5) (see for instance [9], Lemma 2.1).

In the case of the standard sphere, remarkably, an analogue of Theorem 1.1 is available unlike in the case of perturbations of the Yamabe equation when $h_{\delta} \equiv 0$ as investigated in [8, 19]. Here the additional assumption that $\Psi$ in $(1.4)$ has negative average compensates for the vanishing of the Riemannian mass.

Theorem 1.2. Let $\xi_{1,0}$ and $\xi_{2,0}$ be distinct points in $\mathbb{S}^{n}, n \in\{4,5\}$, and define $h_{\delta}$ as in (1.4). Assume in addition that there holds:

$$
\int_{\mathbb{R}^{4}} \Psi(y) d y<0 .
$$

Let $\delta>0$ small be fixed and let $H$ be any function in the class $\mathcal{C}(\mathcal{H})$ defined in (1.7) below. Then, for any $0<\varepsilon \leq \varepsilon_{0}$ small enough, there exists a positive solution $u_{\varepsilon}$ of:

$$
\triangle_{g} u_{\varepsilon}+\left(\frac{n(n-2)}{4}+h_{\delta}+\varepsilon H\right) u_{\varepsilon}=u_{\varepsilon}^{2^{*}-1}
$$

in $M$. This family $\left(u_{\varepsilon}\right)_{0<\varepsilon \leq \varepsilon_{0}}$ blows-up with finite energy at two distincts simple blow-up points as $\varepsilon \rightarrow 0$ and has a zero weak limit.

The families $\left(u_{\varepsilon}\right)_{\varepsilon}$ of positive solutions that we construct in Theorems 1.1 and 1.2 blow up as a sum of two isolated simple bubbles of nonequivalent weights. The highest one concentrates at a point $\xi_{2, \delta}$ satisfying $h_{\delta}\left(\xi_{2, \delta}\right)>0$, while the lowest one concentrates at $\xi_{1,0}$. In our constructions, $\delta$ is fixed small enough so that $h_{\delta}\left(\xi_{1,0}\right)=0$ by (1.4). To our knowledge, Theorems 1.1 and 1.2 yield the first example of multi-bubble configurations in dimensions 4 and 5 when the limiting operator $\triangle_{g}+c_{n} S_{g}+h_{\delta}$ is positive; clustering phenomena in the degenerate case had been previously constructed in [27, 29]. The weak limit of our families $\left(u_{\varepsilon}\right)_{\varepsilon}$ is zero, and this is a necessary condition when $n \in\{4,5\}$ by [5].

A few comments on the choice of $h_{\delta}$ and $H$ are in order here. First, we point out that Theorems 1.1 and 1.2 require no smallness assumption on $h_{\delta}\left(\xi_{2,0}\right)=\Psi(0)$ and therefore yield existence of (blowing-up) positive solutions for model equations like (1.1) when the limiting potential $k$ is allowed to be much larger than $c_{n} S_{g}$ at a blow-up point. Also, in Theorem 1.2, no equivariance assumption is needed on $h_{\delta}$. The class $\mathcal{C}(\mathcal{H})$ of functions $H$ considered in Theorems 1.1 and 1.2 is defined as

$$
\mathcal{C}(\mathcal{H})=\left\{H \in C^{\infty}(M) \text { satisfying }(7.7) \text { and } 7.8 \text { below }\right\} .
$$

These functions $H$ are used to construct the lowest bubble and can be chosen with great generality. By (1.7) we can choose $H \geq 0$, in which case $c_{n} S_{g}+h_{\delta}+\varepsilon H$ in (1.5) (and its counterpart in (1.6)) approaches $c_{n} S_{g}+h_{\delta}$ from above as $\varepsilon \rightarrow 0$. Remark also that since we assumed $\Psi(0)>0$, the limiting potential always satisfies $c_{n} S_{g}+h_{\delta}>c_{n} S_{g}$ at the blow-up point $\xi_{2, \delta}$ (this is proved in Section 7 below). If we moreover assume $\Psi \geq 0$ 
in $\mathbb{R}^{n}$, which is possible in Theorem 1.1, it also satisfies $c_{n} S_{g}+h_{\delta} \geq c_{n} S_{g}$ everywhere in $M$. However it does not satisfy $c_{n} S_{g}+h_{\delta}>c_{n} S_{g}$ everywhere in $M$, in adequation with the results of [5]. Considering the additional $h_{\delta}$ in the potential $c_{n} S_{g}+h_{\delta}$ brings in a new set of technical problems which are not easily dealt with. They arise in Section 7 , where the smallness assumption on $\delta$ is quantified and to which we refer for more details. We should also point out that, in some cases, Theorems 1.1 and 1.2 remain true when $h_{\delta}$ given by (1.4) is replaced by a suitable smooth function $h$. Sufficient conditions on $h$ ensuring this are given in Remark 7.1 below.

Finally, the constructions that we produce here can only occur in dimensions 4 and 5 . Indeed, as a consequence of the 3-dimensional sup-inf inequality, solutions of (1.1) can only blow-up as sums of bubbles of comparable weights when $n=3$ (see [9], Theorem. 5.2 , and see also [10] for other examples of bubbling phenomena in dimension 3). And when $n \geq 7$, as a consequence of [5], energy-bounded families $\left(u_{\varepsilon}\right)_{\varepsilon}$ of solutions of $(1.1)$ only exist if $\lim _{\varepsilon \rightarrow 0} k_{\varepsilon}=c_{n} S_{g}$ at all blow-up points (this remains true if $n=6$ under additional assumptions, see [9] prop. 8.1). Let us also mention that the picture when we drop the bounded-energy assumption is radically different: equivariant infinite-energy solutions when $k>c_{n} S_{g}$ and when $\left(M^{n}, g\right)$ is the standard sphere have been constructed in [4] (when $n \geq 5$ ) and very recently in [31] (when $n=4$ ).

1.2. Strategy of proof of Theorems $\mathbf{1 . 1}$ and $\mathbf{1 . 2}$, First, we explain how an a priori blow-up analysis yields necessary conditions on the bubbling configuration in our setting. Assume that we are given a family $\left(u_{\varepsilon}\right)_{\varepsilon}$ of solutions of $(1.5)($ or $(1.6)$ ) that blows-up with two bubbles - which are not a priori assumed to be isolated. By the $H^{1}$-theory of [26] $u_{\varepsilon}$ writes as

$$
u_{\varepsilon}=W_{1, \varepsilon}+W_{2, \varepsilon}+o(1) \text { in } H^{1}(M),
$$

where $W_{i, \varepsilon}, i=1,2$ are given by $(1.3)$ for some families $\left(\mu_{i, \varepsilon}\right)_{\varepsilon}, i=1,2$ of positive numbers going to 0 and for some families $\left(\xi_{1, \varepsilon}\right)_{\varepsilon}$ and $\left(\xi_{2, \varepsilon}\right)_{\varepsilon}$ of points in $M$ converging towards $\xi_{1}$ and $\xi_{2}$ as $\varepsilon \rightarrow 0$. If we assume now that $h\left(\xi_{2}\right)>0$, there is not much freedom left: there necessarily holds that $h\left(\xi_{1}\right)=0$ and that $\left(\mu_{1, \varepsilon}\right)_{\varepsilon}$ and $\left(\mu_{2, \varepsilon}\right)_{\varepsilon}$ have to satisfy

$$
\begin{array}{ll}
\mu_{1, \varepsilon}=\left(C_{1}+o(1)\right) \mu_{2, \varepsilon} \ln \left(\frac{1}{\mu_{2, \varepsilon}}\right) & \text { if } n=4, \\
\mu_{1, \varepsilon}^{3}=\left(C_{2}+o(1)\right) \mu_{2, \varepsilon} & \text { if } n=5,
\end{array}
$$

as $\varepsilon \rightarrow 0$, for positive constants $C_{1}, C_{2}$. In particular, $\xi_{1}$ and $\xi_{2}$ are distinct and $\xi_{2, \varepsilon}$ is the center of the highest bubble. Similarly, the value of $\mu_{1, \varepsilon}$ is constrained in terms of $\varepsilon$ by:

$$
\begin{array}{ll}
\mu_{1, \varepsilon} \ln \left(\frac{1}{\mu_{1, \varepsilon}}\right)=\left(C_{1}^{\prime}+o(1)\right) \varepsilon & \text { if } n=4, \\
\mu_{1, \varepsilon}=\left(C_{2}^{\prime}+o(1)\right) \varepsilon & \text { if } n=5,
\end{array}
$$

for positive $C_{1}^{\prime}, C_{2}^{\prime}$. We refer to Appendix $\mathrm{A}$ where relations $(1.9)$ and $(1.10)$ are proven.

Relations (1.9) and (1.10) are therefore the starting point of our construction. Equation (1.9) exhibits an asymmetry in the configuration of our bubbles, both in their weights and in the localization of their centers. If we wanted to apply a Lyapunov-Schmidt 
finite-dimensional reduction in $H^{1}(M)$ to produce the constructions of Theorems 1.1 and 1.2 this would force us to work with an extremely high precision, since an expansion of $I_{\varepsilon}\left(W_{1, \varepsilon}+W_{2, \varepsilon}\right)$ involves terms of order $\mu_{2, \varepsilon}^{2}$, where $I_{\varepsilon}$ is the energy functional of 1.5 ) (or (1.6)). When $n=5$, for instance, $\mu_{2, \varepsilon}^{2}$ is comparable to $\mu_{1, \varepsilon}^{6}$ in view of $(1.9)$, which would force us to estimate the $H^{1}(M)$ norm of the error in the nonlinear procedure with a precision $o\left(\mu_{1, \varepsilon}^{3}\right)$. Finding a suitable ansatz for the approximated bubble $W_{1, \varepsilon}$ that both reaches this precision and comes with explicit estimates to be able to compute the additional contributions in $I_{\varepsilon}\left(W_{1, \varepsilon}+W_{2, \varepsilon}\right)$ seems both unnatural and technically out of reach.

We overcome this technical difficulty by combining a priori pointwise asymptotic analysis techniques to a nonlinear finite-dimensional procedure in $H^{1}(M)$. This new approach was recently developed by the first author in [21, 22] to construct instability examples for critical elliptic systems in a coupled supercritical setting. It goes as follows: we first perform the standard nonlinear procedure in $H^{1}(M)$ and construct a candidate solution $W_{1, \varepsilon}+W_{2, \varepsilon}+\phi_{\varepsilon}$ of (1.5) (or (1.6)) up to kernel elements, with $\phi_{\varepsilon}$ controlled in $H^{1}(M)$. We use here the classical Lyapunov-Schmidt approach that has been developed in the last decades, see for instance [16, 20, 23, 25, 32] and the references therein. Since the $H^{1}(M)$ bound on $\phi_{\varepsilon}$ is not precise enough to proceed as usual, we then obtain a thorough pointwise decription of the blow-up behavior of $\phi_{\varepsilon}$ using techniques in the spirit of those developed in [7] and [9. In particular, we do not proceed via an expansion of the reduced-energy in our approach: we conclude our proof by showing that the kernel elements can be annihilated for suitable values of the parameters, and we use for this the latter pointwise estimates on $\phi_{\varepsilon}$.

In view of $(1.9)$, the bubbling configurations that we investigate in this work can be thought of as the low-dimensional counterpart of towering phenomena in higher dimensions. Examples of towering phenomena for positive solutions have recently been constructed in dimensions $n \geq 7$ in [17], carrying out a nice improvement of the usual energy methods, but taking advantage of a radial symmetry assumption. In this respect our approach, which relies on a priori analysis methods to perform the finite-dimensional reduction, allows us to overcome the absence of symmetry in the configuration of our bubbles. We believe our method will prove useful in future work when addressing the construction of involved bubbling configurations, for instance in the absence of symmetries.

The structure of the article is as follows. In Section 2 we introduce the bubbling profiles $W_{1, \varepsilon}$ and $W_{2, \varepsilon}$. An elevated precision is required on $W_{1, \varepsilon}$ while a naive choice of $W_{2, \varepsilon}$ is enough. In Section 3 we apply the standard nonlinear reduction procedure in $H^{1}(M)$ and construct a solution $W_{1, \varepsilon}+W_{2, \varepsilon}+\phi_{\varepsilon}$ of (1.5) up to kernel elements. Sections 4,5 and 6 are the core of the analysis of the paper. In Section 4 we turn the $H^{1}$ bound on $\phi_{\varepsilon}$ into a global $C^{0}$ one and show that $\phi_{\varepsilon}=o\left(W_{1, \varepsilon}+W_{2, \varepsilon}\right)$ in $C^{0}(M)$. This requires an adaptation of the techniques of [7], since $W_{1, \varepsilon}+W_{2, \varepsilon}+\phi_{\varepsilon}$ is only a solution of (1.5) up to kernel elements and can change sign. In Section 5 we improve the global estimate of Section 4 into a sharp higher-order pointwise control on $\phi_{\varepsilon}$ around $\xi_{2}$. This again involves blow-up arguments. Section 6 consists in an asymptotic expansion of the coefficients of the kernel 
elements. On one side, those pertaining to the kernel associated to the lowest bubble $W_{1, \varepsilon}$ are simply expanded using energy estimates. On the other side, those coming from the highest bubble $W_{2, \varepsilon}$ cannot be dealt with in this way and are instead computed using the precise pointwise asymptotics of Section 5. The analysis in Sections 4,5 and 6 does not use (1.4) and can be performed in full generality. Section 7 contains the concluding vanishing argument in the proof of Theorems 1.1 and 1.2 . Finally, Appendix A describes the a priori analysis considerations leading to (1.9).

Acknowledgments: The authors warmly thank Olivier Druet and Emmanuel Hebey for stimulating discussions and valuable comments on the manuscript.

\section{Notations And BUbBling PROFiles}

Let $(M, g)$ be a closed Riemannian manifold of dimension $n \in\{4,5\}$ of positive Yamabe type - that is, such that $\triangle_{g}+c_{n} S_{g}$ is coercive, where $c_{n}=\frac{n-2}{4(n-1)}$ and $S_{g}$ is the scalar curvature of $(M, g)$. By the standard conformal normal coordinates result of Lee-Parker [12], there exists $\Lambda \in C^{\infty}(M \times M)$ such that by letting $\Lambda_{\xi}=\Lambda(\xi, \cdot)$ there holds that:

$$
\Lambda_{\xi}(\xi)=1, \quad \nabla \Lambda_{\xi}(\xi)=0
$$

that

$$
S_{g_{\xi}}(\xi)=0, \quad \nabla S_{g_{\xi}}(\xi)=0, \quad \triangle_{g_{\xi}} S_{g_{\xi}}(\xi)=\frac{1}{6}\left|W_{g}(\xi)\right|_{g}^{2}
$$

where $S_{g_{\xi}}$ denotes the scalar curvature of the conformal metric $g_{\xi}=\Lambda_{\xi}^{\frac{4}{n-2}} g$, and that, for any point $\xi \in M$ there holds for arbitrarily large given $N$ :

$$
\left|\left(\exp _{\xi}^{g_{\xi}}\right)^{*} g_{\xi}\right|(y)=1+O\left(|y|^{N}\right),
$$

$C^{1}$-uniformly in $\xi \in M$ and in $y \in T_{\xi} M,|y| \leq C$. Here $\exp _{\xi}^{g_{\xi}}$ denotes the exponential map for the metric $g_{\xi}$ at $\xi$ with the identification of $T_{\xi} M$ to $\mathbb{R}^{n}$ via a smooth orthonormal basis of $T_{\xi} M$ defined in an open set containing $\xi$. For any $\xi \in M$, we let $G_{g_{\xi}}$ denote the Green's function of the operator $\triangle_{g_{\xi}}+c_{n} S_{g_{\xi}}$ in $M$. Since $n \in\{4,5\}$, the result of [12] asserts that for any $\xi \in M$ one has:

$$
G_{g_{\xi}}\left(\xi, \exp _{\xi}^{g_{\xi}}(y)\right)=\frac{1}{(n-2) \omega_{n-1}}|y|^{2-n}+A(\xi)+O(|y|)
$$

as $|y| \rightarrow 0$, where $\omega_{n-1}$ is the volume of the standard sphere $\mathbb{S}^{n-1}$. The constant $A(\xi)$ in (2.4) is called the mass of $G_{g_{\xi}}$ at $\xi$. It smoothly depends on $\xi$ and there holds $A(\xi)>0$ for any $\xi \in M$ provided $(M, g)$ is not conformally diffeomorphic to the standard sphere, and $A \equiv 0$ otherwise. For the sake of clarity we also recall the conformal covariance property of the conformal laplacian: for any $v \in C^{\infty}(M)$ and $\xi \in M$,

$$
\left(\triangle_{g}+c_{n} S_{g}\right)\left(\Lambda_{\xi} v\right)=\Lambda_{\xi}^{2^{*}-1}\left(\triangle_{g_{\xi}}+c_{n} S_{g_{\xi}}\right)(v)
$$

If $G_{g}$ denotes the Green's function of $\triangle_{g}+c_{n} S_{g}$ in $M$ this yields in particular that :

$$
\text { for any } x \in M, y \in M \backslash\{x\}, \quad G_{g}(x, y)=\Lambda_{\xi}(x) G_{g_{\xi}}(x, y) \Lambda_{\xi}(y) \text {. }
$$


Let $\xi_{1,0}$ and $\xi_{2,0}$ be distinct points of $M$, and let $r_{0}>0$ be such that

$$
8 r_{0}<\min \left(i_{g}(M), d_{g}\left(\xi_{1,0}, \xi_{2,0}\right), \inf _{\xi \in M} d_{g_{\xi}}\left(\xi_{1,0}, \xi_{2,0}\right)\right),
$$

where $i_{g}$ denotes the injectivity radius of $(M, g)$ and $d_{g}$ and $d_{g_{\xi}}$ respectively denote the Riemannian distance associated to the metric $g$ and $g_{\xi}$. Let $H$ and $h$ be smooth functions in $M$. Assume that $H$ is supported in $B_{g_{\xi_{1,0}}}\left(\xi_{1,0}, 2 r_{0}\right)$, where $B_{g_{\xi_{1,0}}}$ denotes the geodesic ball with respect to the metric $g_{\xi_{1,0}}$, that $\triangle_{g}+c_{n} S_{g}+h$ is coercive and that $h$ is supported in $M \backslash B_{g_{\xi_{1,0}}}\left(\xi_{1,0}, 2 r_{0}\right)$, so that the supports of $h$ and $H$ are disjoint. The precise form of $H$ and $h$ will only come into play in Section 7 , and we do not assume for now that (1.4) holds. The blow-up analysis performed in Sections 4 and 5 and the expansions in Section 6 will only rely on the assumption on their supports. Similarly, whether $(M, g)$ is conformally diffeomorphic to the standard sphere or not only comes into play in Section 7 .

Let $\mu_{1}>0$ and $\xi_{1} \in M$. Following [8] we define, for $x \in M$ :

$$
\begin{aligned}
\hat{W}_{1, \mu_{1}, \xi_{1}}(x) & =(n-2) \omega_{n-1} G_{g_{\xi_{1}}}\left(\xi_{1}, x\right) \Lambda_{\xi_{1}}(x) \times \\
& \begin{cases}d_{g_{\xi_{1}}}\left(\xi_{1}, x\right)^{n-2} \mu_{1}^{\frac{n-2}{2}}\left(\mu_{1}^{2}+\frac{d_{g_{\xi_{1}}}\left(\xi_{1}, x\right)^{2}}{n(n-2)}\right)^{1-\frac{n}{2}} & \text { if } d_{g_{\xi_{1}}}\left(\xi_{1}, x\right)<r_{0}, \\
r_{0}^{n-2} \mu_{1}^{\frac{n-2}{2}}\left(\mu_{1}^{2}+\frac{r_{0}^{2}}{n(n-2)}\right)^{1-\frac{n}{2}} & \text { if } d_{g_{\xi_{1}}}\left(\xi_{1}, x\right) \geq r_{0} .\end{cases}
\end{aligned}
$$

For $\mu_{1}>0$ and $\xi_{1} \in M$, let $T_{1, \mu_{1}, \xi_{1}}$ be the unique solution in $M$ of:

$$
\left(\triangle_{g}+\left(c_{n} S_{g}+h\right)\right) T_{1, t_{1}, \xi_{1}}=-h \hat{W}_{1, \mu_{1}, \xi_{1}}
$$

It is a smooth function in $M$ since $h$ is supported in $M \backslash B_{g_{\xi_{1,0}}}\left(\xi_{1,0}, 2 r_{0}\right)$. Let $\chi \in C^{\infty}\left(\mathbb{R}^{+}\right)$ be a smooth nonnegative function, with $\chi \equiv 1$ in $\left[0, r_{0}\right]$ and $\chi \equiv 0$ in $\left[2 r_{0},+\infty\right)$. Define, for $\mu_{1}, \mu_{2}>0, \xi_{1}, \xi_{2} \in M$, and for $x \in M$ :

$$
\begin{aligned}
& W_{1, \mu_{1}, \xi_{1}}(x)=\hat{W}_{1, \mu_{1}, \xi_{1}}(x)+T_{1, \mu_{1}, \xi_{1}}(x), \\
& W_{2, \mu_{2}, \xi_{2}}(x)=\chi\left(d_{g}\left(\xi_{2}, x\right)\right) \mu_{2}^{\frac{n-2}{2}}\left(\mu_{2}^{2}+\frac{d_{g}\left(\xi_{2}, x\right)^{2}}{n(n-2)}\right)^{1-\frac{n}{2}} .
\end{aligned}
$$

As announced in the introduction, the choice of $W_{2, \mu_{2}, \xi_{2}}$ is rougher than the choice of $W_{1, \mu_{1}, \xi_{1}}$; in particular, the conformal correction at $\xi_{2}$ is not required. Note also that $W_{2, \mu_{2}, \xi_{2}}$ is compactly supported in $B_{g}\left(\xi_{2}, 2 r_{0}\right)$. We also define the following approximate 
kernel elements, for $1 \leq j \leq n$ and $x \in M$ :

$$
\begin{aligned}
Z_{1,0, \mu_{1}, \xi_{1}}(x) & =(n-2) \omega_{n-1} d_{g_{\xi_{1}}}\left(\xi_{1}, x\right)^{n-2} G_{g_{\xi_{1}}}\left(\xi_{1}, x\right) \chi\left(d_{g_{\xi_{1}}}\left(\xi_{1}, x\right)\right) \Lambda_{\xi_{1}}(x) \\
& \times \mu_{1}^{\frac{n-2}{2}}\left(\frac{d_{g_{\xi_{1}}}\left(\xi_{1}, x\right)^{2}}{n(n-2)}-\mu_{1}^{2}\right)\left(\mu_{1}^{2}+\frac{d_{g_{\xi_{1}}}\left(\xi_{1}, x\right)^{2}}{n(n-2)}\right)^{-\frac{n}{2}}, \\
Z_{1, j, \mu_{1}, \xi_{1}}(x) & =(n-2) \omega_{n-1} d_{g_{\xi_{1}}}\left(\xi_{1}, x\right)^{n-2} G_{g_{\xi_{1}}}\left(\xi_{1}, x\right) \chi\left(d_{g_{\xi_{1}}}\left(\xi_{1}, x\right)\right) \Lambda_{\xi_{1}}(x) \\
& \times \mu_{1}^{\frac{n}{2}}\left\langle\left(\exp _{\xi_{1}}^{g_{\xi_{1}}}\right)^{-1}(x), e_{j}\left(\xi_{1}\right)\right\rangle_{g_{\xi_{1}}\left(\xi_{1}\right)}\left(\mu_{1}^{2}+\frac{d_{g_{\xi_{1}}}\left(\xi_{1}, x\right)^{2}}{n(n-2)}\right)^{-\frac{n}{2}}, \\
Z_{2,0, \mu_{2}, \xi_{2}} & =\chi\left(d_{g}\left(\xi_{2}, x\right)\right) \mu_{2}^{\frac{n-2}{2}}\left(\frac{d_{g}\left(\xi_{2}, x\right)^{2}}{n(n-2)}-\mu_{2}^{2}\right)\left(\mu_{2}^{2}+\frac{d_{g}\left(\xi_{2}, x\right)^{2}}{n(n-2)}\right)^{-\frac{n}{2}}, \\
Z_{2, j, \mu_{2}, \xi_{2}} & =\chi\left(d_{g}\left(\xi_{2}, x\right)\right) \mu_{2}^{\frac{n}{2}}\left\langle\left(\exp _{\xi_{2}}\right)^{-1}(x), e_{j}\left(\xi_{2}\right)\right\rangle_{g_{\xi_{2}}\left(\xi_{2}\right)}\left(\mu_{2}^{2}+\frac{d_{g}\left(\xi_{2}, x\right)^{2}}{n(n-2)}\right)^{-\frac{n}{2}} .
\end{aligned}
$$

In $(2.10)$ we denoted by the same notation $\left(e_{1}(y), \cdots, e_{n}(y)\right)$ two families of orthonormal vector fields, respectively for $g_{\xi_{1}}$ and $g$, defined in open sets containing respectively $\xi_{1}$ and $\xi_{2}$.

We conclude this subsection with a remark. Let $\xi_{1} \in B_{g_{\xi_{1,0}}}\left(\xi_{1,0}, r_{0}\right)$. By (2.7), and since $h(y)=0$ for any $d_{g_{\xi_{1}}}\left(\xi_{1}, y\right) \leq r_{0}$ by (2.6), $T_{1, \mu_{1}, \xi_{1}}$ in $(2.8)$ is represented, with (2.1), 2.5) and (2.7), as:

$$
\begin{array}{r}
T_{1, \mu_{1}, \xi_{1}}(x)=-(n-2) \omega_{n-1}(n(n-2))^{\frac{n-2}{2}} \mu_{1}^{\frac{n-2}{2}} \int_{M} G_{h}(x, y) h(y) G_{g}\left(y, \xi_{1}\right) d v_{g}(y) \\
+O\left(\mu_{1}^{\frac{n+2}{2}}\right)
\end{array}
$$

where $G_{h}$ denotes the Green's function of $\triangle_{g}+c_{n} S_{g}+h$ in $M$ and the $O\left(\mu_{1}^{\frac{n+2}{2}}\right)$ term is in $C^{2}(M)$ and is independent of the choice of $\mu_{1}$ and $\xi_{1}$. Similarly we also obtain that, for any $y \in M \backslash\left\{\xi_{1}\right\}$ :

$$
G_{g}\left(\xi_{1}, x\right)=G_{h}\left(\xi_{1}, x\right)+\int_{M} G_{g}\left(\xi_{1}, y\right) h(y) G_{h}(y, x) d v_{g}(y) .
$$

The latter with and (2.7), 2.9) and (2.11) shows in particular that, for $d_{g_{\xi_{1}}}\left(\xi_{1}, x\right) \geq r_{0}$, we have:

$$
W_{1, \mu_{1}, \xi_{1}}=(n-2) \omega_{n-1}(n(n-2))^{\frac{n-2}{2}} \mu_{1}^{\frac{n-2}{2}} G_{h}\left(\xi_{1}, \cdot\right)+O\left(\mu_{1}^{\frac{n+2}{2}}\right) \text { in } C^{2}(M) .
$$

\section{Reduced problem in $H^{1}(M)$}

Let $\varepsilon>0$ and let $t_{1}, t_{2}$ be positive numbers. We define:

$$
\mu_{1, \varepsilon}\left(t_{1}\right)=\left\{\begin{array}{ll}
e^{-\frac{t_{1}}{\varepsilon}} & \text { if } n=4 \\
\varepsilon t_{1} & \text { if } n=5
\end{array}, \quad \mu_{2, \varepsilon}\left(t_{1}, t_{2}\right)= \begin{cases}\varepsilon t_{2} e^{-\frac{t_{1}}{\varepsilon}} & \text { if } n=4 \\
\varepsilon^{3} t_{2} & \text { if } n=5 .\end{cases}\right.
$$


As explained in the introduction, this choice of $\mu_{1, \varepsilon}$ and $\mu_{2, \varepsilon}$ is not a lucky guess but is necessary and driven by conditions $(1.9)$ and $(1.10)$. The blowing-up solutions of $(1.5)$ and (1.6) that we construct in this paper are bubbles modeled on 2.9 for the choice of $\mu_{1}, \mu_{2}$ given by (3.1). For $t_{1}, t_{2}>0$ and $\xi_{1}, \xi_{2} \in M$ we thus let, for $0 \leq j \leq n$ :

$$
\begin{aligned}
W_{1, \varepsilon, t_{1}, \xi_{1}} & =W_{1, \mu_{1, \varepsilon}\left(t_{1}\right), \xi_{1}}, \\
W_{2, \varepsilon, t_{1}, t_{2}, \xi_{2}} & =W_{2, \mu_{2, \varepsilon}\left(t_{1}, t_{2}\right), \xi_{2}}, \\
Z_{1, \varepsilon, j, t_{1}, \xi_{1}} & =Z_{1, j, \mu_{1, \varepsilon}\left(t_{1}\right), \xi_{1}}, \\
Z_{2, \varepsilon, j, t_{1}, t_{2}, \xi_{2}} & =Z_{2, \mu_{2, \varepsilon}\left(t_{1}, t_{2}\right), \xi_{2}},
\end{aligned}
$$

where $\mu_{1, \varepsilon}\left(t_{1}\right)$ and $\mu_{2, \varepsilon}\left(t_{1}, t_{2}\right)$ are given by $(3.1)$. Let $A_{0}$ be a connected compact set in $(0,+\infty)$, and let:

$$
A=A_{0} \times \overline{B_{g_{\xi_{1,0}}}\left(\xi_{1,0}, r_{0}\right)} \times A_{0} \times \overline{B_{g}\left(\xi_{2,0}, r_{0}\right)} .
$$

Throughout the paper, for the sake of clarity and since no confusion will occur, whenever $\left(t_{1, \varepsilon}, \xi_{1, \varepsilon}, t_{2, \varepsilon}, \xi_{2, \varepsilon}\right)_{\varepsilon}$ will denote a family of points in $A$, the families $\mu_{1, \varepsilon}\left(t_{1, \varepsilon}\right), \mu_{2, \varepsilon}\left(t_{1, \varepsilon}, t_{2, \varepsilon}\right)$, $W_{1, \varepsilon, t_{1, \varepsilon}, \xi_{1, \varepsilon}}, Z_{1, j, \varepsilon, t_{1}, \xi_{1, \varepsilon}}, W_{2, \varepsilon, t_{1, \varepsilon}, t_{2, \varepsilon}, \xi_{2, \varepsilon}}, Z_{2, j, \varepsilon, t_{1, \varepsilon}, t_{2, \varepsilon}, \xi_{2, \varepsilon}}, 0 \leq j \leq n$ will just be denoted by $\mu_{1}, \mu_{2}, W_{1}, \ldots$. Similarly, $\left(t_{1, \varepsilon}, \xi_{1, \varepsilon}, t_{2, \varepsilon}, \xi_{2, \varepsilon}\right)_{\varepsilon}$ will often simply be denoted by $\left(t_{1}, \xi_{1}, t_{2}, \xi_{2}\right)$. Given $\left(t_{1, \varepsilon}, \xi_{1, \varepsilon}, t_{2, \varepsilon}, \xi_{2, \varepsilon}\right)_{\varepsilon}$, and adopting these notations, we will also let, for any $x \in M$ :

$$
\theta_{1}(x)=\mu_{1}+d_{g_{\xi_{1}}}\left(\xi_{1}, x\right) \text { and } \theta_{2}(x)=\mu_{2}+d_{g}\left(\xi_{2}, x\right)
$$

The points $\xi_{1}$ and $\xi_{2}$ will be thought of as the centers, respectively, of the lowest and the highest bubble. By the choice of $A$ in $(3.3)$, they will always satisfy

$$
d_{g_{\xi_{1,0}}}\left(\xi_{1,0}, \xi_{1}\right) \leq r_{0} \text { and } d_{g}\left(\xi_{2,0}, \xi_{2}\right) \leq r_{0},
$$

so that by 2.6 the supports of $Z_{1, j}$ or $H$ are disjoint from the supports of $W_{2}, Z_{2, k}$ or $h$, for $0 \leq j, k \leq n$. In particular, $W_{2}$ is supported in the region where $h$ is nontrivial.

As a first step of our proof, we apply the standard finite-dimensional reduction scheme to this family of bubbling profiles. For any $\varepsilon>0$ and $\left(t_{1}, \xi_{1}, t_{2}, \xi_{2}\right) \in A$, where $A$ is as in $(3.3)$, let

$$
K_{\varepsilon, t_{1}, \xi_{1}, t_{2}, \xi_{2}}=\operatorname{Span}\left\{Z_{1, j}, Z_{2, k}, 0 \leq j, k \leq n\right\},
$$

where the $Z_{i, j}$ are defined in 3.2 , and let $K_{\varepsilon, t_{1}, \xi_{1}, t_{2}, \xi_{2}}^{\perp}$ be its orthogonal for the scalar product:

$$
\langle u, v\rangle=\int_{M}\left(\langle\nabla u, \nabla v\rangle+\left(c_{n} S_{g}+h+\varepsilon H\right) u v\right) d v_{g} .
$$

In the following, all the $H^{1}(M)$-norms appearing, denoted by $\|\cdot\|_{H^{1}(M)}$, will be taken with respect to this scalar product. Also, throughout this paper, if $\left(f_{\varepsilon}\right)_{\varepsilon},\left(g_{\varepsilon}\right)_{\varepsilon}$ denote families of numbers or functions, the notation " $f_{\varepsilon} \lesssim g_{\varepsilon}$ " will be used to denote the existence of a positive constant $C$ independent of $\varepsilon$ such that $f_{\varepsilon} \leq C g_{\varepsilon}$ for any $\varepsilon$ small enough. If $g_{\varepsilon} \geq 0$, we will also write " $f_{\varepsilon}=O\left(g_{\varepsilon}\right)$ " to say that $\left|f_{\varepsilon}\right| \lesssim g_{\varepsilon}$.

The following result is the starting point of our analysis: 
Proposition 3.1. There exists $\varepsilon_{0}>0$ such that for any $0<\varepsilon \leq \varepsilon_{0}$ and for any $\left(t_{1}, \xi_{1}, t_{2}, \xi_{2}\right) \in A$, there exists $\phi_{\varepsilon}\left(t_{1}, \xi_{1}, t_{2}, \xi_{2}\right) \in K_{\varepsilon, t_{1}, \xi_{1}, t_{2}, \xi_{2}}^{\perp}$ such that

$$
\begin{aligned}
\Pi_{K_{\varepsilon, t_{1}, \xi_{1}, t_{2}, \xi_{2}}^{\perp}} & {\left[W_{1}+W_{2}+\phi_{\varepsilon}\left(t_{1}, \xi_{1}, t_{2}, \xi_{2}\right)\right.} \\
& \left.-\left(\triangle_{g}+c_{n} S_{g}+h+\varepsilon H\right)^{-1}\left(W_{1}+W_{2}+\phi_{\varepsilon}\left(t_{1}, \xi_{1}, t_{2}, \xi_{2}\right)\right)_{+}^{2^{*}-1}\right]=0,
\end{aligned}
$$

where $W_{1}$ and $W_{2}$ are as in (3.2) and where $\Pi_{K_{\varepsilon, t_{1}, \xi_{1}, t_{2}, \xi_{2}}^{\perp}}$ denotes the orthogonal projection on $K_{\varepsilon, t_{1}, \xi_{1}, t_{2}, \xi_{2}}^{\perp}$ for 3.6 . In addition, for any $0<\varepsilon \leq \varepsilon_{0}, \phi_{\varepsilon} \in C^{0}\left(A, H^{1}(M)\right)$ and there exists a positive constant $C$ such that, for any $0<\varepsilon \leq \varepsilon_{0}$ and for any $\left(t_{1, \varepsilon}, \xi_{1, \varepsilon}, t_{2, \varepsilon}, \xi_{2, \varepsilon}\right)_{\varepsilon} \in A$ there holds:

$$
\left\|\phi_{\varepsilon}\left(t_{1, \varepsilon}, \xi_{1, \varepsilon}, t_{2, \varepsilon}, \xi_{2, \varepsilon}\right)\right\|_{H^{1}(M)} \leq C \varepsilon \mu_{1}^{\frac{n-2}{2}},
$$

where $\mu_{1}$ is given by (3.1) for $t_{1}=t_{1, \varepsilon}$. Also, $\phi_{\varepsilon}\left(t_{1}, \xi_{1}, t_{2}, \xi_{2}\right)$ is the unique solution of (3.7) in $K_{\varepsilon, t_{1}, \xi_{1}, t_{2}, \xi_{2}}^{\perp}$ satisfying (3.8).

In (3.7) we have let, for any $u \in H^{1}(M), u_{+}=\max (u, 0)$.

Proof. The existence, continuity and uniqueness properties of $\phi_{\varepsilon}$ for $0<\varepsilon \leq \varepsilon_{0}$ for some $\varepsilon_{0}>0$, as well as (3.7), are a consequence of the general framework developed in [25] (Proposition 5.1), in which (1.5) and (1.6) fall. The result of [25] generalizes previous ideas developed in [16, 20]. It remains to prove (3.8). Let $\left(t_{1, \varepsilon}, \xi_{1, \varepsilon}, t_{2, \varepsilon}, \xi_{2, \varepsilon}\right)_{0<\varepsilon \leq \varepsilon_{0}} \in A$. We claim that the following estimate holds: there exists a positive constant $C$, independent on $\varepsilon$ and on the choice of the family $\left(t_{1, \varepsilon}, \xi_{1, \varepsilon}, t_{2, \varepsilon}, \xi_{2, \varepsilon}\right)_{\varepsilon}$, such that for any $0<\varepsilon \leq \varepsilon_{0}$,

$$
\left\|W_{1}+W_{2}-\left(\triangle_{g}+c_{n} S_{g}+h+\varepsilon H\right)^{-1}\left(W_{1}+W_{2}\right)^{2^{*}-1}\right\|_{H^{1}(M)} \leq C \varepsilon \mu_{1}^{\frac{n-2}{2}}
$$

where we used again the notations $W_{1}, W_{2}, \mu_{1}, \mu_{2}, \xi_{1}, \xi_{2}$ as above. First, a simple test function computation using (3.2), together with Sobolev and trace inequalities shows that

$$
\begin{aligned}
& \left\|W_{1}+W_{2}-\left(\triangle_{g}+c_{n} S_{g}+h+\varepsilon H\right)^{-1}\left(W_{1}+W_{2}\right)^{2^{*}-1}\right\|_{H^{1}(M)} \\
& \quad \lesssim\left\|\left(\triangle_{g}+c_{n} S_{g}+h+\varepsilon H\right)\left(W_{1}+W_{2}\right)-\left(W_{1}+W_{2}\right)^{2^{*}-1}\right\|_{L^{\frac{2 n}{n+2}}(M)} \\
& \quad+\left\|\partial_{\text {in }} W_{1}+\partial_{\text {out }} W_{1}\right\|_{L} L^{\frac{2(n-1)}{n}}\left(\partial B_{g_{\xi_{1}}}\left(\xi_{1}, r_{0}\right)\right)
\end{aligned}
$$

where $\partial_{\text {in }} W_{1}$ and $\partial_{\text {out }} W_{1}$ denote the derivative with respect to the unit outward and inward normal for $g_{\xi_{1}}$ to $\partial B_{g_{\xi_{1}}}\left(\xi_{1}, r_{0}\right)$. By 3.2 and 2.8 and since the supports of $W_{2}$ 
and $H$ are disjoint we write, in $M \backslash \partial B_{g_{\xi_{1}}}\left(\xi_{1}, r_{0}\right)$, that:

$$
\begin{aligned}
\left(\triangle_{g}+\right. & \left.c_{n} S_{g}+h+\varepsilon H\right)\left(W_{1}+W_{2}\right)-\left(W_{1}+W_{2}\right)^{2^{*}-1}=\left(\triangle_{g}+c_{n} S_{g}+\varepsilon H\right) \hat{W}_{1}-\hat{W}_{1}^{2^{*}-1} \\
& +\varepsilon H T_{1}+\hat{W}_{1}^{2^{*}-1}-\left(\hat{W}_{1}+T_{1}\right)^{2^{*}-1}+\left(\triangle_{g}+c_{n} S_{g}+h\right) W_{2}-W_{2}^{2^{*}-1} \\
& +W_{1}^{2^{*}-1}+W_{2}^{2^{*}-1}-\left(W_{1}+W_{2}\right)^{2^{*}-1} .
\end{aligned}
$$

On one side, straightforward computations using (2.11) and 3.2 give:

$$
\begin{aligned}
& \left\|\varepsilon H T_{1}+\hat{W}_{1}^{2^{*}-1}-\left(\hat{W}_{1}+T_{1}\right)^{2^{*}-1}+W_{1}^{2^{*}-1}+W_{2}^{2^{*}-1}-\left(W_{1}+W_{2}\right)^{2^{*}-1}\right\|_{L^{\frac{2 n}{n+2}}(M)} \\
& \lesssim \varepsilon \mu_{1}^{\frac{n-2}{2}}+\mu_{1}^{n-2}+\left(\mu_{1} \mu_{2}\right)^{\frac{n-2}{2}} .
\end{aligned}
$$

On the other side, straightforward computations give that there holds:

$$
\left|\partial_{\text {in }} W_{1}+\partial_{\text {out }} W_{1}\right| \lesssim \mu_{1}^{\frac{n+2}{2}} \text { in } \partial B_{g_{\xi_{1}}}\left(\xi_{1}, r_{0}\right)
$$

and that, both in $B_{g_{\xi_{1}}}\left(\xi_{1}, r_{0}\right)$ and in $M \backslash \overline{B_{g_{\xi_{1}}}\left(\xi_{1}, r_{0}\right)}$, there holds:

$$
\left|\left(\triangle_{g}+c_{n} S_{g}+\varepsilon H\right) \hat{W}_{1}-\hat{W}_{1}^{2^{*}-1}\right| \lesssim \mu_{1}^{\frac{n+2}{2}} r_{1}^{n-4}\left(\mu_{1}^{2}+\frac{r_{1}^{2}}{n(n-2)}\right)^{-\frac{n}{2}},
$$

where we have let $r_{1}=d_{g_{\xi_{1}}}\left(\xi_{1}, \cdot\right)$ (see for instance [8], Proposition 2.2). As a consequence:

$$
\left\|\left(\triangle_{g}+c_{n} S_{g}+\varepsilon H\right) \hat{W}_{1}-\hat{W}_{1}^{2^{*}-1}\right\|_{L^{\frac{2 n}{n+2}(M)}}+\left\|\partial_{i n} W_{1}+\partial_{\text {out }} W_{1}\right\|_{L^{\frac{2(n-1)}{n}}\left(\partial B_{g_{\xi_{1}}}\left(\xi_{1}, r_{0}\right)\right)} \lesssim \varepsilon \mu_{1}^{\frac{n-2}{2}} .
$$

Finally, straightforward computations using (3.2) show that there holds, for any $x \in M$ :

$$
\left|\left(\triangle_{g}+c_{n} S_{g}+h\right) W_{2}(x)-W_{2}^{2^{*}-1}(x)\right| \lesssim \mu_{2}^{\frac{n-2}{2}} \theta_{2}(x)^{2-n},
$$

where $\theta_{2}$ is defined in (3.4). This gives in the end:

$$
\left\|\left(\triangle_{g}+c_{n} S_{g}+h\right) W_{2}(x)-W_{2}^{2^{*}-1}(x)\right\|_{L^{\frac{2 n}{n+2}(M)}} \lesssim \mu_{2}^{\frac{n-2}{2}} .
$$

Combining all these computations into (3.10) and using the explicit expression of $\mu_{1}, \mu_{2}$ given by (3.1) concludes the proof of (3.9). Estimate (3.8) then follows from (3.9) by the result of $[25]$.

\section{4. $C^{0}$-THEORY AND UNIFORM a priori POINTWISE ESTIMATES ON $\phi_{\varepsilon}$}

Let $\varepsilon_{0}$ be given by Proposition 3.1. For $0<\varepsilon \leq \varepsilon_{0}$, let $\left(t_{1}, \xi_{1}, t_{2}, \xi_{2}\right) \in A$ and let $\phi_{\varepsilon}=\phi_{\varepsilon}\left(t_{1}, \xi_{1}, t_{2}, \xi_{2}\right)$ be given by Proposition 3.1. Equation (3.7) shows that there exist $\lambda_{i, j}^{\varepsilon}=\lambda_{i, j}^{\varepsilon}\left(t_{1}, \xi_{1}, t_{2}, \xi_{2}\right), i=1,2,0 \leq j \leq n$, such that $\phi_{\varepsilon}$ satisfies:

$$
\left(\triangle_{g}+c_{n} S_{g}+h+\varepsilon H\right) u_{\varepsilon}=\left(u_{\varepsilon}\right)_{+}^{2^{*}-1}+\sum_{i, j} \lambda_{i, j}^{\varepsilon}\left(\triangle_{g}+c_{n} S_{g}+h+\varepsilon H\right) Z_{i, j}
$$

where we have let:

$$
u_{\varepsilon}=u_{\varepsilon, t_{1}, \xi_{1}, t_{2}, \xi_{2}}=W_{1}+W_{2}+\phi_{\varepsilon},
$$


and $W_{1}=W_{1, \varepsilon, t_{1}, \xi_{1}}, \ldots$ are given by (3.2). Since $\phi_{\varepsilon} \in K_{\varepsilon, t_{1}, \xi_{1}, t_{2}, \xi_{2}}^{\perp}$, integrating 4.1 against $Z_{i, j}$ for $1 \leq i \leq 2$ and $0 \leq j \leq n$ and using (3.8) yields the existence of a positive constant $C$ such that for any $0<\varepsilon \leq \varepsilon_{0}$ and for any $\left(t_{1, \varepsilon}, \xi_{1, \varepsilon}, t_{2, \varepsilon}, \xi_{2, \varepsilon}\right)_{\varepsilon \leq \varepsilon_{0}} \in A$ there holds, for all $1 \leq i \leq 2$ and $0 \leq j \leq n$ :

$$
\left|\lambda_{i, j}^{\varepsilon}\right|=\left|\lambda_{i, j}^{\varepsilon}\left(t_{1, \varepsilon}, \xi_{1, \varepsilon}, t_{2, \varepsilon}, \xi_{2, \varepsilon}\right)\right| \leq C \varepsilon \mu_{1}^{\frac{n-2}{2}} .
$$

We aim at constructing a solution of (1.5) and (1.6) via (4.1) by finding an element $\left(t_{1, \varepsilon}, \xi_{1, \varepsilon}, t_{2, \varepsilon}, \xi_{2, \varepsilon}\right)_{\varepsilon}$ which annihilates all the $\lambda_{i, j}^{\varepsilon}$. This goes through an asymptotic expansion in $C^{0}(A)$ of the $\lambda_{i, j}^{\varepsilon}$ as $\varepsilon \rightarrow 0$, where $A$ is given in (3.3). However, as explained in the introduction, having $h>0$ in the region where the center of the highest bubble $W_{2}$ is expected to be localized requires these expansions to be carried out with a high precision that cannot be reached with the mere $H^{1}$ estimate $(3.8)$. In this section we therefore obtain a priori global pointwise asymptotic estimates on $\phi_{\varepsilon}$. These will be refined into sharp second-order estimates on $\phi_{\varepsilon}$ around $\xi_{2}$ in the next section.

We show that $\phi_{\varepsilon}$ is, in a pointwise sense, globally small compared to $W_{1}$ and $W_{2}$ :

Proposition 4.1. There exists $\varepsilon_{1}>0$ and a family of positive numbers $\left(\nu_{\varepsilon}\right)_{0<\varepsilon \leq \varepsilon_{1}}$ with $\lim _{\varepsilon \rightarrow 0} \nu_{\varepsilon}=0$ such that there holds, for any $0<\varepsilon \leq \varepsilon_{1}$ and for any $\left(t_{1}, \xi_{1}, t_{2}, \xi_{2}\right) \in A$ :

$$
\left|\phi_{\varepsilon}\left(t_{1}, \xi_{1}, t_{2}, \xi_{2}\right)(x)\right| \leq \nu_{\varepsilon}\left(W_{1}(x)+W_{2}(x)\right) \quad \text { for any } x \in M \text {. }
$$

Here again $W_{1}$ and $W_{2}$ are given by (3.2) and $A$ is as in (3.3). In particular, up to assuming that $\varepsilon_{1}$ is small enough, we will assume that $\nu_{\varepsilon} \leq \frac{1}{2}$ for $0<\varepsilon \leq \varepsilon_{1}$.

Proof of Proposition 4. The proof of Proposition 4 is divided into two Lemmas. The first one establishes, for a fixed $\varepsilon$, continuity properties of the mapping $\phi_{\varepsilon}$ in strong spaces.

Lemma 4.2. There exists $\varepsilon_{1}>0$ such that, for any $0<\varepsilon \leq \varepsilon_{1}$ the mappings:

$$
\begin{aligned}
& \left(t_{1}, \xi_{1}, t_{2}, \xi_{2}\right) \in A \longmapsto W_{1, \varepsilon, t_{1}, \xi_{1}}+W_{2, \varepsilon, t_{1}, t_{2}, \xi_{2}} \in C^{0}(M) \\
& \left(t_{1}, \xi_{1}, t_{2}, \xi_{2}\right) \in A \longmapsto \phi_{\varepsilon}\left(t_{1}, \xi_{1}, t_{2}, \xi_{2}\right) \in C^{0}(M)
\end{aligned}
$$

are well-defined and continuous.

Proof. For the first map, the assertion simply follows from the explicit expression of the right-hand side given by $(3.2)$ and by the regularity properties of $\Lambda_{\xi_{1}}$. We thus prove the Lemma for the second map. First, by (3.1) and (3.8) we let $\varepsilon_{1}>0$ be such that, for any $0<\varepsilon \leq \varepsilon_{1}$ and any $\left(t_{1}, \xi_{1}, t_{2}, \xi_{2}\right) \in A$ there holds:

$$
\left\|\phi_{\varepsilon}\left(t_{1}, \xi_{1}, t_{2}, \xi_{2}\right)\right\|_{H^{1}(M)}<\frac{1}{2} K_{n}^{-\frac{n-2}{2}}
$$

where we have let:

$$
K_{n}=\sqrt{\frac{4}{n(n-2) \omega_{n}^{\frac{2}{n}}}}
$$

and $\omega_{n}$ is the volume of the standard unit $n$-sphere. Let $0<\varepsilon \leq \varepsilon_{1}$ be fixed and $\left(t_{1}, \xi_{1}, t_{2}, \xi_{2}\right) \in A$. First, since $Z_{i, j}$ is smooth for any $i \in\{1,2\}$ and $j \in\{0, \cdots, n\}$, by 
(4.1) and by an adaptation of Trudinger's argument [30] (see also [9], Theorem 2.15) we get that

$$
\left(W_{1}+W_{2}+\phi_{\varepsilon}\right)_{+} \in L^{s}(M)
$$

for some $s>2^{*}$. Then, with (4.1), a bootstrap procedure applies and shows that $u_{\varepsilon} \in C^{2}(M)$, and hence that $\phi_{\varepsilon} \in C^{0}(M)$. Note however that $\phi_{\varepsilon}$ is not smooth on the sphere $\left\{d_{g_{\xi_{1,0}}}\left(\xi_{1,0}, y\right)=r_{0}\right\}$ since $\hat{W}_{1}$ is not.

Let now $\left(t_{1, k}, \xi_{1, k}, t_{2, k}, \xi_{2, k}\right)_{k \geq 1}$ be a sequence of points of $A$ converging towards $\left(t_{1,0}, \xi_{1,0}, t_{2,0}, \xi_{2,0}\right)$ and let $\phi_{k}=\phi_{\varepsilon}\left(t_{1, k}, \xi_{1, k}, t_{2, k}, \xi_{2, k}\right)$ for any $k \geq 1$ and $\phi_{0}=\phi_{\varepsilon}\left(t_{1,0}, \xi_{1,0}, t_{2,0}, \xi_{2,0}\right)$. By Proposition 3.1, $\phi_{k} \rightarrow \phi_{0}$ in $H^{1}(M)$ as $k \rightarrow+\infty$. Assume first that the sequence $\left(\phi_{k}\right)_{k}$ is uniformly (in $k$ ) bounded in $L^{\infty}(M)$. Then (3.2), (4.3) and standard elliptic theory in (4.1) show that every subsequence of $\left(\phi_{k}\right)_{k}$ admits a subsequence which converges in $C^{0}(M)$, and therefore to $\phi_{0}$. In this case, thus, $\phi_{k} \rightarrow \phi_{0}$ in $C^{0}(M)$ as $k \rightarrow+\infty$.

We therefore assume that, up to a subsequence, $\left\|\phi_{k}\right\|_{L^{\infty}(M)} \rightarrow+\infty$ as $k \rightarrow+\infty$. A Green's representation formula for $u_{k}$ given by (4.2) with (4.1) and standard properties of Green's functions (see [24]) show, since $\left(W_{1}+W_{2}+\phi_{k}\right)_{+} \geq 0$, that there exists a positive constant $C_{\varepsilon}$, independent of $k$, such that:

$$
\inf _{M} \phi_{k} \geq-C_{\varepsilon}
$$

for any $k \geq 1$. In particular, we might as well assume that $\max _{M}\left(\phi_{k}\right)_{+} \rightarrow+\infty$ as $k \rightarrow+\infty$ and let $x_{k}$ be such that $\phi_{k}\left(x_{k}\right)=\left\|\phi_{k}\right\|_{L^{\infty}(M)}=\max _{M}\left(\phi_{k}\right)_{+} \rightarrow+\infty$ as $k \rightarrow+\infty$. We let $\mu_{k}=\phi_{k}\left(x_{k}\right)^{-\frac{2}{n-2}}$ and, for any $x \in B_{0}\left(i_{g}(M) / \mu_{k}\right)$, we let $g_{k}=\exp _{x_{k}}^{*} g\left(\mu_{k} \cdot\right)$ and

$$
\tilde{u}_{k}(x)=\mu_{k}^{\frac{n-2}{2}} u_{k}\left(\exp _{x_{k}}\left(\mu_{k} x\right)\right) .
$$

With (4.1), $\tilde{u}_{k}$ satisfies, for any $y \in B_{0}\left(i_{g}(M) / \mu_{k}\right)$ :

$$
\begin{aligned}
\triangle_{g_{k}} \tilde{u}_{k}(y) & +\mu_{k}^{2}\left(c_{n} S_{g}+h+\varepsilon H\right)\left(y_{k}\right) \tilde{u}_{k}(y)=\left(\tilde{u}_{k}(y)\right)_{+}^{2^{*}-1} \\
& +\mu_{k}^{\frac{n+2}{2}} \sum_{i, j} \lambda_{i, j}^{\varepsilon}\left(t_{1, k}, \xi_{1, k}, t_{2, k}, \xi_{2, k}\right)\left(\triangle_{g}+c_{n} S_{g}+h+\varepsilon H\right) Z_{i, j}\left(y_{k}\right),
\end{aligned}
$$

where we have let $y_{k}=\exp _{x_{k}}\left(\mu_{k} y\right)$. By (4.7), by the definition of $x_{k}$ and since $\varepsilon$ is fixed throughout this proof there holds:

$$
-C_{\varepsilon} \mu_{k}^{\frac{n-2}{2}} \leq \tilde{u}_{k}(y) \leq 1+C_{0} \mu_{k}^{\frac{n-2}{2}}
$$

for some positive constant $C_{0}$ and for any $y \in B_{0}\left(i_{g}(M) / \mu_{k}\right)$. By $(4.3)$ and standard elliptic theory, $\tilde{u}_{k}$ converges therefore in $C_{l o c}^{1}\left(\mathbb{R}^{n}\right)$ to $\tilde{u}_{0}$, with $0 \leq \tilde{u}_{0} \leq 1$, solution of

$$
\triangle_{\xi} \tilde{u}_{0}=\tilde{u}_{0}^{2^{*}-1} \text {. }
$$

By the definition of $x_{k}$ we also have $\tilde{u}_{0}(0)=1$, so that the classification result in [3] implies that $\left\|\tilde{u}_{0}\right\|_{L^{2^{*}\left(\mathbb{R}^{n}\right)}}=K_{n}^{-\frac{n-2}{2}}$, where $K_{n}$ is given by 4.6$)$. For a given $R>0$ there 
holds thus:

$$
\begin{aligned}
\int_{B_{x_{k}}\left(R \mu_{k}\right)}\left|\phi_{k}\right|^{2^{*}} d v_{g} & \geq \int_{B_{x_{k}}\left(R \mu_{k}\right)}\left|u_{k}\right|^{2^{*}} d v_{g}+O\left(\mu_{k}^{\frac{n-2}{2}}\right) \\
& =\int_{B_{0}(R)}\left|\tilde{u}_{k}\right|^{2^{*}} d v_{g_{k}}+O\left(\mu_{k}^{\frac{n-2}{2}}\right) \\
& =\int_{B_{0}(R)}\left|\tilde{u}_{0}\right|^{2^{*}} d x+o(1)=\left(1+\varepsilon_{R}\right) K_{n}^{-n}+o(1),
\end{aligned}
$$

as $k \rightarrow+\infty$, where $\lim _{R \rightarrow+\infty} \varepsilon_{R}=0$. This is a contradiction with 4.5 for $R$ and $k$ large enough and concludes the proof of the Claim.

The second Lemma establishes a rough version of (4.4):

Lemma 4.3. Let $\varepsilon_{1}$ be as in Lemma 4.2 and let $\left(t_{1, \varepsilon}, \xi_{1, \varepsilon}, t_{2, \varepsilon}, \xi_{2, \varepsilon}\right)_{0<\varepsilon \leq \varepsilon_{1}}$ be a family of points of $A$. Define, for any $0<\varepsilon \leq \varepsilon_{1}$ :

$$
\nu_{\varepsilon}:=\left\|\frac{\phi_{\varepsilon}\left(t_{1, \varepsilon}, \xi_{1, \varepsilon}, t_{2, \varepsilon}, \xi_{2, \varepsilon}\right)}{W_{1, \varepsilon, t_{1, \varepsilon}, \xi_{1, \varepsilon}}+W_{2, \varepsilon, t_{1, \varepsilon}, t_{2, \varepsilon}, \xi_{2, \varepsilon}}}\right\|_{C^{0}(M)} .
$$

Then $\nu_{\varepsilon} \rightarrow 0$ as $\varepsilon \rightarrow 0$.

Proof. We prove Lemma 4.3 by contradiction, and therefore assume the existence of a sequence $\left(\varepsilon_{k}\right)_{k}, 0<\varepsilon_{k} \leq \varepsilon_{1}$, with $\varepsilon_{k} \rightarrow 0$ as $k \rightarrow+\infty$, such that

$$
\left\|\frac{\phi_{k}}{W_{1}+W_{2}}\right\|_{C^{0}(M)} \geq \eta_{0}
$$

for some $\eta_{0}>0$, for all $k \geq 1$. In (4.8), for the sake of simplicity and using the previous notations, we simply wrote $t_{1, \varepsilon_{k}}=t_{1}, \xi_{1, \varepsilon_{k}}=\xi_{1}, W_{1, \varepsilon_{k}, t_{1, \varepsilon_{k}}, \xi_{1, \varepsilon_{k}}}=W_{1}$, $\phi_{\varepsilon_{k}}\left(t_{1, k}, \xi_{1, k}, t_{2, k}, \xi_{2, k}\right)=\phi_{k}$ and so on. We will keep these notations throughout the proof of the Lemma and it will be implicit that we will be working with the quantities given by (3.2), associated to the sequences $\left(\varepsilon_{k}\right)_{k}$ and $\left(t_{1, \varepsilon_{k}}, \xi_{1, \varepsilon_{k}}, t_{2, \varepsilon_{k}}, \xi_{2, \varepsilon_{k}}\right)_{k}$.

The proof of Lemma 4.3 consists in an asymptotic a priori analysis of the sequence $\left(\phi_{k}\right)_{k}$ and is divided into several steps.

Step 1: local convergence. We first show that, for $1 \leq i \leq 2$, there holds, up to a subsequence:

$$
\mu_{i}^{\frac{n-2}{2}} u_{k}\left(\exp _{\xi_{i}}\left(\mu_{i} \cdot\right)\right) \rightarrow U_{0} \quad \text { in } C_{l o c}^{1}\left(\mathbb{R}^{n}\right)
$$

as $k \rightarrow+\infty$, where $u_{k}=u_{\varepsilon_{k}}$ is as in (4.2), where $\mu_{1}, \mu_{2}$ are given by (3.1) and

$$
U_{0}(x)=\left(1+\frac{|x|^{2}}{n(n-2)}\right)^{1-\frac{n}{2}} \quad \text { for } x \in \mathbb{R}^{n} .
$$

The arguments involved in the proof of (4.9) are slightly more complicated than in the usual cases since $u_{k}$ can change sign. For $1 \leq i \leq 2$ and for $x \in B_{0}\left(i_{g}(M) / \mu_{i}\right)$, let 
$v_{i, k}(x)=\mu_{i}^{\frac{n-2}{2}} u_{k}\left(\exp _{\xi_{i}}\left(\mu_{i} x\right)\right)$. Letting $g_{i, k}=\exp _{\xi_{i}}^{*} g\left(\mu_{i} \cdot\right)$, with 4.1 $v_{i, k}$ satisfies, for any $x \in B_{0}\left(i_{g}(M) / \mu_{i}\right)$ and for $x_{k}=\exp _{\xi_{i}}\left(\mu_{i} x\right)$ :

$$
\begin{aligned}
\triangle_{g_{i, k}} v_{i, k}(x) & +\mu_{i}^{2}\left(c_{n} S_{g}+h+\varepsilon_{k} H\right)\left(x_{k}\right) v_{i, k}=\left(v_{i, k}\right)_{+}^{2^{*}-1} \\
& +\sum_{i, j} \lambda_{i, j}^{\varepsilon_{k}} \mu_{i}^{\frac{n+2}{2}}\left(\triangle_{g}+c_{n} S_{g}+h+\varepsilon_{k} H\right) Z_{i, j}\left(x_{k}\right) .
\end{aligned}
$$

We show that $v_{i, k}$ is uniformly bounded in $C_{l o c}^{0}\left(\mathbb{R}^{n}\right)$ by investigating its positive and negative part separately. First, a straightforward adaptation of the arguments in [18] shows, with 4.11), that $\left(v_{i, k}\right)_{+}=\max \left(v_{i, k}, 0\right)$ satisfies the following equation in a weak sense:

$$
\begin{aligned}
\triangle_{g_{i, k}}\left(v_{i, k}\right)_{+} & +\mu_{i}^{2}\left(c_{n} S_{g}+h+\varepsilon_{k} H\right)\left(\exp _{\xi_{i}}\left(\mu_{i} \cdot\right)\right)\left(v_{i, k}\right)_{+} \leq\left(v_{i, k}\right)_{+}^{2^{*}-1} \\
& +\sum_{i, j} \lambda_{i, j}^{\varepsilon_{k}} \mu_{i}^{\frac{n+2}{2}}\left(\triangle_{g}+c_{n} S_{g}+h+\varepsilon_{k} H\right) Z_{i, j}\left(\exp _{\xi_{i}}\left(\mu_{i} \cdot\right)\right) \mathbb{1}_{v_{i, k}>0} .
\end{aligned}
$$

By 4.3 and 2.10 we have:

$$
\sum_{i, j} \lambda_{i, j}^{\varepsilon_{k}} \mu_{i}^{\frac{n+2}{2}}\left(\triangle_{g}+c_{n} S_{g}+h+\varepsilon_{k} H\right) Z_{i, j}\left(x_{k}\right) \rightarrow 0 \quad \text { in } C_{l o c}^{0}\left(\mathbb{R}^{n}\right) \text { as } k \rightarrow+\infty .
$$

Also, $\mu_{i}^{2}\left(c_{n} S_{g}+h+\varepsilon_{k} H\right)\left(\exp _{\xi_{i}}\left(\mu_{i} \cdot\right)\right) \rightarrow 0$ in $C_{l o c}^{0}\left(\mathbb{R}^{n}\right)$, and by the definition of $v_{i, k}$, by (3.8) and (4.2) there holds that:

$$
\lim _{r \rightarrow 0} \limsup _{k \rightarrow+\infty} \int_{B_{x}(r)} v_{i, k}^{2^{*}}(y) d y=0 \quad \forall x \in \mathbb{R}^{n} .
$$

Hence, an adaptation of Trudinger's argument [30] to 4.12 shows that for any $R>0$ there exists $C_{R}>0$ such that

$$
\left\|\left(v_{i, k}\right)_{+}\right\|_{C^{0}\left(B_{0}(R)\right)} \leq C_{R}
$$

for $k$ large enough.

Independently, let $G_{k}$ denote the Green's function of $\triangle_{g}+\left(c_{n} S_{g}+h+\varepsilon_{k} H\right)$ in $M$ and let $\left(x_{k}\right)_{k}$ be a sequence of points in $M$. By (3.2), 4.1) and (4.3) a representation formula for $u_{k}$ gives:

$$
u_{k}\left(x_{k}\right) \gtrsim-\varepsilon_{k} \mu_{1}^{\frac{n-2}{2}}\left(W_{1}\left(x_{k}\right)+W_{2}\left(x_{k}\right)\right) .
$$

We used here that by $(2.10)$ and $(3.2)$ there exists a positive constant $C$ depending only on $n$ such that $\left|Z_{i, j}\right| \leq C W_{i}$ for $i=1,2$ and $j=0, \ldots, n$. Since by (2.6) and (3.3) there holds $\lim \inf _{k \rightarrow+\infty} d_{g}\left(\xi_{1}, \xi_{2}\right)>0$, the latter inequality shows in particular that for any $x \in B_{0}\left(i_{g}(M) / \mu_{i}\right)$ :

This shows that:

$$
v_{i, k} \gtrsim-\varepsilon_{k}-\varepsilon_{k} \mu_{1}^{n-2} \mu_{2}^{\frac{n-2}{2}}
$$

$$
\left(v_{i, k}\right)_{-} \rightarrow 0 \quad \text { in } C_{l o c}^{0}\left(\mathbb{R}^{n}\right) \text { as } k \rightarrow+\infty .
$$

Standard elliptic theory, with (4.13) and (4.14), shows with (4.11) that $v_{i, k}$ converges in $C_{l o c}^{1}\left(\mathbb{R}^{n}\right)$, up to a subsequence, as $k \rightarrow+\infty$. And (3.1), (3.2), (3.8) and (4.2) show that the limit is $U_{0}$ given in (4.10), which concludes the proof of 4.9$)$. 
Step 2: uniform lower bound on $\phi_{k}$. We now show that there exists a positive sequence $\eta_{k} \rightarrow 0$ as $k \rightarrow+\infty$ such that, up to a subsequence,

$$
\phi_{k}(x) \gtrsim-\eta_{k}\left(W_{1}+W_{2}\right)(x) \quad \text { for any } x \in M \text {. }
$$

Let $\left(x_{k}\right)_{k}$ a sequence of points such that

$$
\frac{\phi_{k}}{W_{1}+W_{2}}\left(x_{k}\right)=\inf _{x \in M} \frac{\phi_{k}}{W_{1}+W_{2}} .
$$

Remember that $W_{1}+W_{2}$ is positive in $M$. We write again a representation formula for $u_{k}$ with 4.1) and (4.3), which gives:

$$
\begin{aligned}
\left(W_{1}+W_{2}\right. & \left.+\phi_{k}\right)\left(x_{k}\right) \\
& \gtrsim \int_{M} G_{k}\left(x_{k}, y\right)\left(W_{1}+W_{2}+\phi_{k}\right)_{+}^{2^{*}-1} d v_{g}-\varepsilon_{k} \mu_{1}^{\frac{n-2}{2}}\left(W_{1}+W_{2}\right) \\
& \gtrsim-\varepsilon_{k} \mu_{1}^{\frac{n-2}{2}}\left(W_{1}+W_{2}\right)+\int_{B_{\xi_{1}}\left(R_{k} \mu_{1}\right)} G_{\varepsilon}\left(x_{k}, y\right)\left(W_{1}+W_{2}+\phi_{k}\right)_{+}^{2^{*}-1} d v_{g} \\
& +\int_{B_{\xi_{2}}\left(R_{k} \mu_{2}\right)} G_{k}\left(x_{k}, y\right)\left(W_{1}+W_{2}+\phi_{k}\right)_{+}^{2^{*}-1} d v_{g},
\end{aligned}
$$

where $R_{k}>0$ is chosen so that $R_{k} \mu_{i} \rightarrow 0$ as $k \rightarrow+\infty$ for $i=1,2$ and such that $B_{\xi_{1}}\left(R_{k} \mu_{1}\right)$ and $B_{\xi_{2}}\left(R_{k} \mu_{2}\right)$ are disjoint for all $k$. The integrals in (4.17) are estimated with Fatou's lemma and (4.9) which in turn, with (4.16), yields 4.15).

Step 3: Blow-up analysis. Step 2 shows in particular that, for $k$ large enough,

$$
u_{k}=W_{1}+W_{2}+\phi_{k} \geq \frac{1}{2}\left(W_{1}+W_{2}\right) \text { in } M .
$$

So $u_{k}$ actually solves, in $M$

$$
\left(\triangle_{g}+c_{n} S_{g}+h+\varepsilon_{k} H\right) u_{k}=u_{k}^{2^{*}-1}+\sum_{i, j} \lambda_{i, j}^{\varepsilon_{k}}\left(\triangle_{g}+c_{n} S_{g}+h+\varepsilon_{k} H\right) Z_{i, j} .
$$

Using (4.3), an adaptation of the blow-up analysis performed in [22] (Proposition 4.1, Steps $4,5,6)$, see also [28], shows that there holds, for any sequence $\left(x_{k}\right)_{k}$ of points of $M$ :

$$
\left|\phi_{k}\left(x_{k}\right)\right|=o\left(W_{1}\left(x_{k}\right)+W_{2}\left(x_{k}\right)\right) .
$$

Applying the latter to the sequence $\left(x_{k}\right)_{k}$ that achieves the maximum point of $\frac{\phi_{k}}{W_{1}+W_{2}}$ in $M$ then yields a contradiction with (4.8), and concludes the proof of Lemma 4.3

We now conclude the proof of Proposition 4.1. Let $\varepsilon_{1}$ be as in Lemma 4.2. Then, again by Lemma 4.2 . for any $0<\varepsilon \leq \varepsilon_{1}$ there exists $\left(t_{1, \varepsilon}, \xi_{1, \varepsilon}, t_{2, \varepsilon}, \xi_{2, \varepsilon}\right) \in A$ such that:

$$
\left\|\frac{\phi_{\varepsilon}\left(t_{1, \varepsilon}, \xi_{1, \varepsilon}, t_{2, \varepsilon}, \xi_{2, \varepsilon}\right)}{W_{1, \varepsilon, t_{1, \varepsilon}, \xi_{1, \varepsilon}}+W_{2, \varepsilon, t_{1, \varepsilon}, t_{2, \varepsilon}, \xi_{2, \varepsilon}}}\right\|_{C^{0}(M)}=\sup _{\left(t_{1}, \xi_{1}, t_{2}, \xi_{2}\right) \in A}\left\|\frac{\phi_{\varepsilon}\left(t_{1}, \xi_{1}, t_{2}, \xi_{2}\right)}{W_{1, \varepsilon, t_{1}, \xi_{1}}+W_{2, \varepsilon, t_{1}, t_{2}, \xi_{2}}}\right\|_{C^{0}(M)},
$$


where $A$ is as in (3.3). Let, for any $0<\varepsilon \leq \varepsilon_{1}, \nu_{\varepsilon}$ be given by Lemma 4.3 for this maximal family $\left(t_{1, \varepsilon}, \xi_{1, \varepsilon}, t_{2, \varepsilon}, \xi_{2, \varepsilon}\right)_{0<\varepsilon \leq \varepsilon_{1}}$. Then, for any $x \in M$, for any $0<\varepsilon \leq \varepsilon_{1}$ and for any $\left(t_{1}, \xi_{1}, t_{2}, \xi_{2}\right) \in A$, there holds by (4.18) that:

$$
\frac{\left|\phi_{\varepsilon}\left(t_{1}, \xi_{1}, t_{2}, \xi_{2}\right)(x)\right|}{W_{1, \varepsilon, t_{1}, \xi_{1}}(x)+W_{2, \varepsilon, t_{1}, t_{2}, \xi_{2}}(x)} \leq \nu_{\varepsilon}
$$

Since $\lim _{\varepsilon \rightarrow 0} \nu_{\varepsilon}=0$, this proves (4.4) and concludes the proof of Proposition 4.1.

The estimates on $\left.\mid \phi_{\varepsilon}\left(t_{1}, \xi_{1}, t_{2}, \xi_{2}\right)\right) \mid$ given by Proposition 4.1 are, for a given $\varepsilon$, uniform in the choice of $\left(t_{1}, \xi_{1}, t_{2}, \xi_{2}\right)$. This is an important property of our analysis that will be crucial in the final argument of the proof of Theorems 1.1 and 1.2 .

A consequence of Proposition 4.1 is that for any $0<\varepsilon \leq \varepsilon_{1}$ and for any $\left(t_{1}, \xi_{1}, t_{2}, \xi_{2}\right) \in A$, we now have

$$
W_{1}+W_{2}+\phi_{\varepsilon}\left(t_{1}, \xi_{1}, t_{2}, \xi_{2}\right) \geq \frac{1}{2}\left(W_{1}+W_{2}\right) .
$$

In particular, with (4.1), we now see that for any $0<\varepsilon \leq \varepsilon_{1}$ and for any $\left(t_{1}, \xi_{1}, t_{2}, \xi_{2}\right)$, $u_{\varepsilon}$ given by 4.2 actually satisfies in $M$ :

$$
\left(\triangle_{g}+c_{n} S_{g}+h+\varepsilon H\right) u_{\varepsilon}=u_{\varepsilon}^{2^{*}-1}+\sum_{i, j} \lambda_{i, j}^{\varepsilon}\left(\triangle_{g}+c_{n} S_{g}+h+\varepsilon H\right) Z_{i, j} .
$$

\section{SECOND-ORDER POINTWISE ESTIMATES}

In this section we refine the pointwise estimate on $\phi_{\varepsilon}$ given by Proposition 4.1 in balls of fixed radius centered at $\xi_{2}$. These improved pointwise estimates will compensate for the insufficient precision of (3.8) and will be the crucial ingredient of the asymptotic expansion of the $\lambda_{i, j}^{\varepsilon}$ in Section 6. Let:

$$
A_{1}=A_{0} \times \overline{B_{g_{\xi_{1,0}}}\left(\xi_{1,0}, r_{0}\right)} \times A_{0} \times \overline{B_{g}\left(\xi_{2,0}, \frac{r_{0}}{2}\right)},
$$

where $A_{0}$ is the compact set in $(0,+\infty)$ appearing in $(3.3)$. The second-order estimates that we obtain are as follows:

Proposition 5.1. There exists $\varepsilon_{2}>0$ and $C>0$ such that, for any $0<\varepsilon \leq \varepsilon_{2}$ and for any family $\left(t_{1, \varepsilon}, \xi_{1, \varepsilon}, t_{2, \varepsilon}, \xi_{2, \varepsilon}\right)_{0<\varepsilon \leq \varepsilon_{2}} \in A_{1}$, where $A_{1}$ is defined in (5.1), we have:

- If $n=4$, and for any $x \in B_{g}\left(\xi_{2, \varepsilon}, \frac{r_{0}}{2}\right)$ :

$$
\left|\phi_{\varepsilon}(x)\right| \leq C\left(\nu_{\varepsilon}\left(\mu_{1}+\mu_{2}\right)+\mu_{2}\left|\ln \theta_{2}(x)\right|+\mu_{1} \mu_{2}\left|\ln \left(\frac{\theta_{2}(x)}{\mu_{2}}\right)\right| \frac{\mu_{2}}{\theta_{2}(x)^{2}}\right),
$$

- If $n=5$, and for any $x \in B_{g}\left(\xi_{2, \varepsilon}, \frac{r_{0}}{2}\right)$ :

$$
\left|\phi_{\varepsilon}(x)\right| \leq C\left(\nu_{\varepsilon}\left(\mu_{1}^{\frac{3}{2}}+\mu_{2}^{\frac{3}{2}}\right)+\mu_{2}^{\frac{3}{2}} \theta_{2}(x)^{-1}+\mu_{1}^{\frac{7}{2}}+\mu_{1}^{\frac{3}{2}}\left|\ln \mu_{2}\right| \mu_{2}^{\frac{3}{2}}\left(\frac{\mu_{2}}{\theta_{2}(x)^{2}}\right)^{\frac{3}{2}}\right) .
$$

Here, as before, we have let $\phi_{\varepsilon}=\phi_{\varepsilon}\left(t_{1, \varepsilon}, \xi_{1, \varepsilon}, t_{2, \varepsilon}, \xi_{2, \varepsilon}\right), \mu_{1}$ and $\mu_{2}$ are given by (3.1), $\theta_{2}$ is as in (3.4) and $\nu_{\varepsilon}$ is given by Proposition 4.1.

As for the proof of Proposition 4.1, we start by proving a weaker version of the result. 
Lemma 5.2. Let $\varepsilon_{1}$ and $\left(\nu_{\varepsilon}\right)_{0<\varepsilon \leq \varepsilon_{1}}$ be given by Proposition 4.1 and let $\left(t_{1, \varepsilon}, \xi_{1, \varepsilon}, t_{2, \varepsilon}, \xi_{2, \varepsilon}\right)_{0<\varepsilon \leq \varepsilon_{1}}$ be a family in $A$, where $A$ is in (3.3). There exists $0<\varepsilon_{2} \leq \varepsilon_{1}$ and $C>0$ such that:

- If $n=4$, and for any $x \in B_{g}\left(\xi_{2, \varepsilon}, 2 r_{0}\right)$ :

$$
\left|\phi_{\varepsilon}(x)\right| \leq C\left(\nu_{\varepsilon}\left(\mu_{1}+\mu_{2}\right)+\mu_{2}\left|\ln \theta_{2}(x)\right|+\mu_{1} \mu_{2}\left|\ln \left(\frac{\theta_{2}(x)}{\mu_{2}}\right)\right| \frac{\mu_{2}}{\theta_{2}(x)^{2}}\right),
$$

- If $n=5$, and for any $x \in B_{g}\left(\xi_{2, \varepsilon}, 2 r_{0}\right)$ :

$$
\left|\phi_{\varepsilon}(x)\right| \leq C\left(\nu_{\varepsilon}\left(\mu_{1}^{\frac{3}{2}}+\mu_{2}^{\frac{3}{2}}\right)+\mu_{2}^{\frac{3}{2}} \theta_{2}(x)^{-1}+\mu_{1}^{\frac{7}{2}}+\mu_{1}^{\frac{3}{2}}\left|\ln \mu_{2}\right| \mu_{2}^{\frac{3}{2}}\left(\frac{\mu_{2}}{\theta_{2}(x)^{2}}\right)^{\frac{3}{2}}\right),
$$

where we used the same notations as in the statement of Proposition 5.1.

Proof. Let $\left(t_{1, \varepsilon}, \xi_{1, \varepsilon}, t_{2, \varepsilon}, \xi_{2, \varepsilon}\right)_{0<\varepsilon \leq \varepsilon_{1}} \in A$. Throughout the rest of this proof $C$ will denote a positive constant independent of $\varepsilon$, which might change from one line to another. We will adopt the same notations as before.

We first assume that $n=4$. By 4.19 and since the supports of $H$ and $W_{2}$ are disjoint, $\phi_{\varepsilon}=\phi_{\varepsilon}\left(t_{1, \varepsilon}, \xi_{1, \varepsilon}, t_{2, \varepsilon}, \xi_{2, \varepsilon}\right)$ satisfies in $M$ :

$$
\begin{aligned}
& \left(\triangle_{g}+\frac{1}{6} S_{g}+h+\varepsilon H\right)\left(\phi_{\varepsilon}-\sum_{i, j} \lambda_{i, j}^{\varepsilon} Z_{i, j}\right)=\left(W_{1}+W_{2}+\phi_{\varepsilon}\right)^{3}-\left(W_{1}+W_{2}\right)^{3} \\
& -\left[\left(\triangle_{g}+\frac{1}{6} S_{g}+h+\varepsilon H\right) W_{1}-W_{1}^{3}\right]+3 W_{1}^{2} W_{2}+3 W_{1} W_{2}^{2} \\
& -\left[\left(\triangle_{g}+\frac{1}{6} S_{g}+h\right) W_{2}-W_{2}^{3}\right] .
\end{aligned}
$$

For any $0<\varepsilon \leq \varepsilon_{1}$, let $G_{\varepsilon}$ be the Green's function of $\triangle_{g}+\frac{1}{6} S_{g}+h$ with Dirichlet boundary condition on $B_{g}\left(\xi_{2}, 2 r_{0}\right)$ (remember that $\left.\xi_{2}=\xi_{2, \varepsilon}\right)$. Let $\left(x_{\varepsilon}\right)_{\varepsilon \leq \varepsilon_{1}}$ be a family of points in $B_{g}\left(\xi_{2}, 2 r_{0}\right)$. For any $0<\varepsilon \leq \varepsilon_{1}$, if $x_{\varepsilon} \in B_{g}\left(\xi_{2}, 2 r_{0}\right) \backslash B_{g}\left(\xi_{2}, r_{0}\right)$, there holds trivially:

$$
\left|\phi_{\varepsilon}\left(x_{\varepsilon}\right)\right| \leq\left\|\phi_{\varepsilon}\right\|_{C^{0}\left(2 r_{0} \backslash r_{0}\right)}
$$

where we have let $\left\|\phi_{\varepsilon}\right\|_{C^{0}\left(2 r_{0} \backslash r_{0}\right)}=\left\|\phi_{\varepsilon}\right\|_{C^{0}\left(B_{g}\left(\xi_{2}, 2 r_{0}\right) \backslash B_{g}\left(\xi_{2}, r_{0}\right)\right)}$. Otherwise we write a representation formula on $B_{g}\left(\xi_{2}, 2 r_{0}\right)$ for $\phi_{\varepsilon}$ with (5.6). This is possible since by definition $W_{1}$ is smooth in $B_{g}\left(\xi_{2}, 2 r_{0}\right)$ and therefore so is $\phi_{\varepsilon}$. Since the $Z_{1, j}$ and $H$ are supported outside of $B_{\xi_{2}}\left(2 r_{0}\right)$ we get that:

$\left|\phi_{\varepsilon}\left(x_{\varepsilon}\right)-\sum_{j=0}^{4} \lambda_{2, j}^{\varepsilon} Z_{2, j}\left(x_{\varepsilon}\right)\right| \leq C\left(\left\|\phi_{\varepsilon}\right\|_{C^{0}\left(2 r_{0} \backslash r_{0}\right)}+\left|\lambda_{2,0}^{\varepsilon}\right| \mu_{2}+\sum_{j=1}^{4}\left|\lambda_{2, j}^{\varepsilon}\right| \mu_{2}^{2}+I_{1}+I_{2}+I_{3}+I_{4}\right)$, 
where

$$
\begin{aligned}
& I_{1}=\int_{B_{g}\left(\xi_{2}, 2 r_{0}\right)} G_{\varepsilon}\left(x_{\varepsilon}, y\right)\left|\left(W_{1}+W_{2}+\phi_{\varepsilon}\right)^{3}(y)-\left(W_{1}+W_{2}\right)^{3}(y)\right| d v_{g}(y), \\
& I_{2}=\int_{B_{g}\left(\xi_{2}, 2 r_{0}\right)} G_{\varepsilon}\left(x_{\varepsilon}, y\right)\left|\left(\triangle_{g}+\frac{1}{6} S_{g}+h\right) W_{1}-W_{1}^{3}\right| d v_{g}(y), \\
& I_{3}=\int_{B_{g}\left(\xi_{2}, 2 r_{0}\right)} G_{\varepsilon}\left(x_{\varepsilon}, y\right)\left(W_{1}^{2} W_{2}+W_{1} W_{2}^{2}\right) d v_{g}(y), \\
& I_{4}=\int_{B_{g}\left(\xi_{2}, 2 r_{0}\right)} G_{\varepsilon}\left(x_{\varepsilon}, y\right)\left|\left(\triangle_{g}+\frac{1}{6} S_{g}+h\right) W_{2}-W_{2}^{3}\right| d v_{g}(y) .
\end{aligned}
$$

First, by (3.12) and standard properties of Green functions (see [24]) there holds:

$$
I_{4} \leq C \mu_{2}\left|\ln \theta_{2}\left(x_{\varepsilon}\right)\right|,
$$

where $\theta_{2}$ is as in (3.4). Then, by (3.2) and since by 2.6) there holds $d_{g_{\xi_{1}}}\left(\xi_{1}, y\right)>r_{0}$ for any $y \in B_{\xi_{2}}\left(2 r_{0}\right)$, it is easily seen that there holds:

$$
I_{2} \leq C \mu_{1}^{3} \text {. }
$$

Straightforward computations using (3.2) show that there holds:

$$
I_{3} \leq C\left(\mu_{2}\left|\ln \theta_{2}\left(x_{\varepsilon}\right)\right|+\mu_{1} \mu_{2}\left|\ln \left(\frac{\theta_{2}\left(x_{\varepsilon}\right)}{\mu_{2}}\right)\right| \frac{\mu_{2}}{\theta_{2}\left(x_{\varepsilon}\right)^{2}}\right) .
$$

Finally, Proposition 4.1 shows that

$$
\left|\left(W_{1}+W_{2}+\phi_{\varepsilon}\right)^{3}-\left(W_{1}+W_{2}\right)^{3}\right| \leq C\left(W_{1}+W_{2}\right)^{2}\left|\phi_{\varepsilon}\right|
$$

so that straightforward computations lead to:

$$
I_{1} \leq C\left(\mu_{1}^{2}+\mu_{2}\left|\ln \left(\frac{\theta_{2}\left(x_{\varepsilon}\right)}{\mu_{2}}\right)\right| \frac{\mu_{2}}{\theta_{2}\left(x_{\varepsilon}\right)^{2}}\right)\left\|\phi_{\varepsilon}\right\|_{C^{0}\left(2 r_{0}\right)},
$$

where we have let $\left\|\phi_{\varepsilon}\right\|_{C^{0}\left(2 r_{0}\right)}=\left\|\phi_{\varepsilon}\right\|_{C^{0}\left(B_{\xi_{2}}\left(2 r_{0}\right)\right)}$. Combining (5.9)-5.12) in (5.8) gives, with (5.7) and (3.1), that there holds, for any $\left(x_{\varepsilon}\right)_{0<\varepsilon \leq \varepsilon_{1}}, x_{\varepsilon} \in B_{g}\left(\xi_{2}, 2 r_{0}\right)$ :

$$
\begin{aligned}
\mid \phi_{\varepsilon}\left(x_{\varepsilon}\right)- & \sum_{j=0}^{4} \lambda_{2, j}^{\varepsilon} Z_{2, j}\left(x_{\varepsilon}\right) \mid \leq C\left(\left\|\phi_{\varepsilon}\right\|_{C^{0}\left(2 r_{0} \backslash r_{0}\right)}+\left|\lambda_{2,0}^{\varepsilon}\right| \mu_{2}+\sum_{j=1}^{4}\left|\lambda_{2, j}^{\varepsilon}\right| \mu_{2}^{2}+\mu_{2}\left|\ln \theta_{2}\left(x_{\varepsilon}\right)\right|\right. \\
& \left.+\left(\mu_{1}^{2}+\mu_{2}\left|\ln \left(\frac{\theta_{2}\left(x_{\varepsilon}\right)}{\mu_{2}}\right)\right| \frac{\mu_{2}}{\theta_{2}\left(x_{\varepsilon}\right)^{2}}\right)\left\|\phi_{\varepsilon}\right\|_{C^{0}\left(2 r_{0}\right)}+\mu_{1} \mu_{2}\left|\ln \left(\frac{\theta_{2}\left(x_{\varepsilon}\right)}{\mu_{2}}\right)\right| \frac{\mu_{2}}{\theta_{2}\left(x_{\varepsilon}\right)^{2}}\right) .
\end{aligned}
$$

By evaluating (5.13) at suitable points satisfying $d_{g}\left(x_{\varepsilon}, \xi_{2}\right) \leq \mu_{2}$ one gets with (3.1) the following estimate on the $\lambda_{2, j}^{\varepsilon}, 0 \leq j \leq 4$ :

$$
\sum_{j=0}^{4}\left|\lambda_{2, j}^{\varepsilon}\right| \leq C \mu_{2}\left(\left\|\phi_{\varepsilon}\right\|_{C^{0}\left(2 r_{0}\right)}+\mu_{1}\right),
$$


so that, using Proposition 4.1, (5.13) improves into:

$$
\begin{aligned}
\left|\phi_{\varepsilon}\left(x_{\varepsilon}\right)\right| & \leq C\left(\nu_{\varepsilon}\left(\mu_{1}+\mu_{2}\right)+\mu_{2}\left|\ln \theta_{2}\left(x_{\varepsilon}\right)\right|\right. \\
& \left.+\left(\mu_{1}^{2}+\mu_{2}\left|\ln \left(\frac{\theta_{2}\left(x_{\varepsilon}\right)}{\mu_{2}}\right)\right| \frac{\mu_{2}}{\theta_{2}\left(x_{\varepsilon}\right)^{2}}\right)\left\|\phi_{\varepsilon}\right\|_{C^{0}\left(2 r_{0}\right)}+\mu_{1} \mu_{2}\left|\ln \left(\frac{\theta_{2}\left(x_{\varepsilon}\right)}{\mu_{2}}\right)\right| \frac{\mu_{2}}{\theta_{2}\left(x_{\varepsilon}\right)^{2}}\right) .
\end{aligned}
$$

We now claim that the following result holds true:

Claim 5.3. There exists $0<\varepsilon_{2} \leq \varepsilon_{1}$ and $C>0$ such that, for any $0<\varepsilon \leq \varepsilon_{2}$, there holds:

$$
\left\|\phi_{\varepsilon}\right\|_{C^{0}\left(2 r_{0}\right)} \leq C \mu_{1} .
$$

Proof. Remember that $\mu_{1}=\mu_{1, \varepsilon}\left(t_{1, \varepsilon}\right)$ is given by (3.1). We proceed by contradiction and assume that for some sequence $\left(\varepsilon_{k}\right)_{k}$ of positive numbers, $\varepsilon_{k} \rightarrow 0$ as $k \rightarrow+\infty$, there holds

$$
\left\|\phi_{k}\right\|_{C^{0}\left(2 r_{0}\right)} \gg \mu_{1} \text { as } k \rightarrow+\infty \text {. }
$$

As before, until the end of this Claim it will be implicit that all the quantities $\mu_{1}, \xi_{1}, \ldots$ depend on this subsequence $\left(\varepsilon_{k}\right)_{k}$ according to (3.1) and (3.2). We will let in particular $\phi_{k}=\phi_{\varepsilon_{k}}\left(t_{1}, \xi_{1}, t_{2}, \xi_{2}\right)$. Let $\left(y_{k}\right)_{k}$ be a sequence of points such that $\left|\phi_{k}\left(y_{k}\right)\right|=\left\|\phi_{k}\right\|_{C^{0}\left(2 r_{0}\right)}$. By (3.1) and (5.17) there holds

$$
\mu_{2}\left|\ln \theta_{2}\left(y_{k}\right)\right| \leq \mu_{2}\left|\ln \mu_{2}\right|=o\left(\left\|\phi_{k}\right\|_{C^{0}\left(2 r_{0}\right)}\right)
$$

as $k \rightarrow+\infty$, and by Proposition $4.1 \lim _{k \rightarrow+\infty} \nu_{\varepsilon_{k}}=0$ so that (5.15) and (5.17) show that

$$
\theta_{2}\left(y_{k}\right) \leq C \mu_{2},
$$

where $\theta_{2}$ is defined in $(3.4)$. For any $y \in B_{0}\left(\frac{r_{0}}{\mu_{2}}\right)$ we then let:

$$
\tilde{\phi}_{k}(y)=\frac{1}{\left\|\phi_{k}\right\|_{C^{0}\left(2 r_{0}\right)}} \phi_{k}\left(\exp _{\xi_{2}}\left(\mu_{2} y\right)\right) .
$$

On one hand, (5.15) and (5.17) show that:

$$
\left|\tilde{\phi}_{k}(y)\right| \leq C \frac{\ln (1+|y|)}{(1+|y|)^{2}}+o(1) \text { on } B_{0}\left(\frac{r_{0}}{\mu_{2}}\right) .
$$

On the other hand, (3.2), 3.11), 3.12 and (5.17) show that:

$$
\begin{array}{r}
\frac{\mu_{2}^{2}}{\left\|\phi_{k}\right\|_{C^{0}\left(2 r_{0}\right)}}\left(-\left[\left(\triangle_{g}+\frac{1}{6} S_{g}+h\right) W_{1}-W_{1}^{3}\right]+3 W_{1}^{2} W_{2}+3 W_{1} W_{2}^{2}\right. \\
\left.-\left[\left(\triangle_{g}+\frac{1}{6} S_{g}+h\right) W_{2}-W_{2}^{3}\right]\right)\left(\exp _{\xi_{2}}\left(\mu_{2} \cdot\right)\right)
\end{array}
$$

converges to 0 in $C_{l o c}^{0}\left(\mathbb{R}^{4}\right)$ as $k$ goes to $+\infty$. Using Proposition 4.1, (5.6) and standard elliptic theory, we then get that $\tilde{\phi}_{k}$ converges in $C_{l o c}^{1}\left(\mathbb{R}^{4}\right)$, up to a subsequence, towards 
$\tilde{\phi}_{0}$ as $k \rightarrow+\infty$, where $\tilde{\phi}_{0}$ solves in $\mathbb{R}^{4}$ :

$$
\triangle_{\text {eucl }} \tilde{\phi}_{0}=3 U_{0}^{2} \tilde{\phi}_{0}+\sum_{j=0}^{4} \tilde{\lambda}_{2, j}^{0} V_{j}
$$

Here eucl denotes the Euclidean metric, $U_{0}$ is as in 4.10 and for any $0 \leq j \leq 4$ we have let:

$$
\tilde{\lambda}_{2, j}^{0}=\lim _{k \rightarrow+\infty} \frac{\lambda_{2, j}^{\varepsilon_{k}}}{\mu_{2}\left\|\phi_{k}\right\|_{C^{0}\left(2 r_{0}\right)}} .
$$

That this limit exists, up to a subsequence, is a consequence of (5.14) and (5.17). Also, in (5.21), $V_{j}$ is defined in $\mathbb{R}^{n}$ for any $n \geq 3$ by:

$$
\begin{aligned}
& V_{0}(y)=\left(\frac{|y|^{2}}{n(n-2)}-1\right)\left(1+\frac{|y|^{2}}{n(n-2)}\right)^{-\frac{n}{2}}, \\
& V_{j}(y)=y_{j}\left(1+\frac{|y|^{2}}{n(n-2)}\right)^{-\frac{n}{2}}, \quad \text { for any } 1 \leq j \leq n .
\end{aligned}
$$

Passing (5.20) to the limit shows that $\tilde{\phi}_{0} \in L^{4}\left(\mathbb{R}^{4}\right)$ so that integrating (5.21) against $V_{j}$ shows that $\tilde{\lambda}_{2, j}^{0}=0$ for any $0 \leq j \leq 4$. Now the result of [1] implies that:

$$
\tilde{\phi}_{0} \in \operatorname{Vect}\left\{V_{j}, 0 \leq j \leq 4\right\} \text {. }
$$

To conclude the proof of Claim 5.3 we now prove that $\tilde{\phi}_{0} \in \operatorname{Vect}\left\{V_{j}, 0 \leq j \leq 4\right\}^{\perp}$, where the orthogonal is taken for the usual scalar product in $\dot{H}^{1}\left(\mathbb{R}^{4}\right)$. This will imply that $\tilde{\phi}_{0} \equiv 0$, which is a contradiction since $\left|\tilde{\phi}_{0}\left(\tilde{y}_{0}\right)\right|=1$ by $(5.18)$, where $\tilde{y}_{0}$ is the limit of $\frac{1}{\mu_{2}} \exp _{\xi_{2}}^{-1}\left(y_{k}\right)$ as $k \rightarrow+\infty$.

To prove that $\tilde{\phi}_{0} \in \operatorname{Vect}\left\{V_{j}, 0 \leq j \leq 4\right\}^{\perp}$, we write that by Proposition 3.1 there holds, for any $0 \leq j \leq 4$ :

$$
\left\langle\phi_{k}, Z_{j}\right\rangle=0
$$

where $\langle\cdot, \cdot\rangle$ is the scalar product given by (3.6). Let now $R>0$ be fixed. The latter equality implies, since $Z_{2, j}, 0 \leq j \leq n$, is supported in $B_{g}\left(\xi_{2}, 2 r_{0}\right)$, that:

$$
\begin{aligned}
\int_{B_{g}\left(\xi_{2}, R \mu_{2}\right)}\left(\left\langle\nabla \phi_{k}, \nabla Z_{2, j}\right\rangle+\right. & \left.\left(\frac{1}{6} S_{g}+h\right) \phi_{k} Z_{2, j}\right) d v_{g}=\int_{\partial B_{g}\left(\xi_{2}, R \mu_{2}\right)} \partial_{\nu} Z_{2, j} \phi_{k} d \sigma_{g} \\
& -\int_{B_{g}\left(\xi_{2}, 2 r_{0}\right) \backslash B_{g}\left(\xi_{2}, R \mu_{2}\right)}\left(\triangle_{g}+\frac{1}{6} S_{g}+h\right) Z_{2, j} \phi_{k} d v_{g} .
\end{aligned}
$$

Straightforward computations using 2.10 show that there holds, for $y \in B_{g}\left(\xi_{2}, 2 r_{0}\right)$ :

$$
\begin{aligned}
& \left|\triangle_{g} Z_{2,0}(y)-3 W_{2}(y)^{2} Z_{2,0}(y)\right| \leq C \mu_{2} \theta_{2}(y)^{-2}, \\
& \left|\triangle_{g} Z_{2, j}(y)-3 W_{2}(y)^{2} Z_{2, j}(y)\right| \leq C \mu_{2}^{2} \theta_{2}(y)^{-3}, \text { for } 1 \leq j \leq 4,
\end{aligned}
$$

where $\theta_{2}$ is as in (3.4). Now, (3.2) and (5.15) show that:

$$
\left|\int_{\partial B_{g}\left(\xi_{2}, R \mu_{2}\right)} \partial_{\nu} Z_{2, j} \phi_{k} d \sigma_{g}\right| \leq\left(C \frac{\ln (1+R)}{1+R}+o(1)\right) \mu_{2}\left\|\phi_{k}\right\|_{C^{0}\left(2 r_{0}\right)},
$$


that

$$
\left|\int_{B_{g}\left(\xi_{2}, 2 r_{0}\right) \backslash B_{g}\left(\xi_{2}, R \mu_{2}\right)} \mu_{2} \theta_{2}(\cdot)^{-2} \phi_{k} d v_{g}\right|=o\left(\mu_{2}\left\|\phi_{k}\right\|_{C^{0}\left(2 r_{0}\right)}\right)
$$

and that

$$
\left|\int_{B_{g}\left(\xi_{2}, 2 r_{0}\right) \backslash B_{g}\left(\xi_{2}, R \mu_{2}\right)} W_{2}^{2} Z_{2, j} \phi_{k} d v_{g}\right| \leq\left(\frac{C}{(1+R)^{3}}+o(1)\right) \mu_{2}\left\|\phi_{k}\right\|_{C^{0}\left(2 r_{0}\right)},
$$

so that $(5.23)$ becomes:

$$
\left|\int_{B_{g}\left(\xi_{2}, R \mu_{2}\right)}\left(\left\langle\nabla \phi_{k}, \nabla Z_{2, j}\right\rangle+\left(\frac{1}{6} S_{g}+h\right) \phi_{k} Z_{2, j}\right) d v_{g}\right| \leq\left(C \frac{\ln (1+R)}{1+R}+o(1)\right) \mu_{2}\left\|\phi_{k}\right\|_{C^{0}\left(2 r_{0}\right)} .
$$

Dividing both sides by $\mu_{2}\left\|\phi_{k}\right\|_{C^{0}\left(2 r_{0}\right)}$, using the definition of $\tilde{\phi}_{k}$ in $(5.19)$, letting first $k$ go to $+\infty$ and then $R \rightarrow+\infty$ we obtain that:

$$
\int_{\mathbb{R}^{4}}\left\langle\nabla \tilde{\phi}_{0}, \nabla V_{j}\right\rangle d x=0
$$

This proves that $\tilde{\phi}_{0} \in \operatorname{Vect}\left\{V_{j}, 0 \leq j \leq 4\right\}^{\perp}$ and, as explained above, gives a contradiction, thus concluding the proof of Claim 5.3 .

Plugging (5.16) into (5.15) and using (3.1) concludes the proof of Lemma 5.2 for $n=4$.

Assume now that $n=5$. The proof is similar to the four-dimensional case. By 4.19) $\phi_{\varepsilon}$ satisfies in $M$ :

$$
\begin{aligned}
& \left(\triangle_{g}+\frac{3}{16} S_{g}+h+\varepsilon H\right)\left(\phi_{\varepsilon}-\sum_{i, j} \lambda_{i, j}^{\varepsilon} Z_{i, j}\right)=\left(W_{1}+W_{2}+\phi_{\varepsilon}\right)^{\frac{7}{3}}-\left(W_{1}+W_{2}\right)^{\frac{7}{3}} \\
& +\left(W_{1}+W_{2}\right)^{\frac{7}{3}}-W_{1}^{\frac{7}{3}}-W_{2}^{\frac{7}{3}}-\left[\left(\triangle_{g}+\frac{3}{16} S_{g}+h+\varepsilon H\right) W_{1}-W_{1}^{\frac{7}{3}}\right] \\
& -\left[\left(\triangle_{g}+\frac{3}{16} S_{g}+h\right) W_{2}-W_{2}^{\frac{7}{3}}\right] .
\end{aligned}
$$

For any $0<\varepsilon \leq \varepsilon_{1}$, let $G_{\varepsilon}$ be the Green's function of $\triangle_{g}+\frac{3}{16} S_{g}+h$ with Dirichlet boundary condition on $B_{g}\left(\xi_{2}, 2 r_{0}\right)$. Let $\left(x_{\varepsilon}\right)_{0<\varepsilon \leq \varepsilon_{1}}$ be any family of points in $B_{g}\left(\xi_{2}, 2 r_{0}\right)$. For any $0<\varepsilon \leq \varepsilon_{1}$, if $x_{\varepsilon} \in B_{g}\left(\xi_{2}, 2 r_{0}\right) \backslash B_{g}\left(\xi_{2}, r_{0}\right)$, there holds trivially:

$$
\left|\phi_{\varepsilon}\left(x_{\varepsilon}\right)\right| \leq\left\|\phi_{\varepsilon}\right\|_{C^{0}\left(2 r_{0} \backslash r_{0}\right)} .
$$

Otherwise we write a representation formula for $\phi_{\varepsilon}$ with 5.25 . Since the $Z_{1, j}$ are supported outside of $B_{g}\left(\xi_{2}, 2 r_{0}\right)$ mimicking the computations that led to (5.13) and 
using (4.3), we get that there holds, for any $\left(x_{\varepsilon}\right)_{0<\varepsilon \leq \varepsilon_{1}}, x_{\varepsilon} \in B_{g}\left(\xi_{2}, 2 r_{0}\right)$ :

$$
\begin{aligned}
& \left|\phi_{\varepsilon}\left(x_{\varepsilon}\right)-\sum_{j=0}^{5} \lambda_{2, j}^{\varepsilon} Z_{2, j}\left(x_{\varepsilon}\right)\right| \leq C\left(\left\|\phi_{\varepsilon}\right\|_{C^{0}\left(2 r_{0} \backslash r_{0}\right)}+\mu_{1}^{\frac{7}{2}}+\mu_{2}^{\frac{3}{2}} \theta_{2}\left(x_{\varepsilon}\right)^{-1}+\mu_{1}^{\frac{3}{2}}\left(\frac{\mu_{2}}{\theta_{2}\left(x_{\varepsilon}\right)}\right)^{2}\right. \\
& \left.+\left(\mu_{1}^{2}+\left(\frac{\mu_{2}}{\theta_{2}\left(x_{\varepsilon}\right)}\right)^{2}\right)\left\|\phi_{\varepsilon}\right\|_{C^{0}\left(2 r_{0}\right)}\right) .
\end{aligned}
$$

Evaluating again the latter estimate at suitable points satisfying $d_{g}\left(x_{\varepsilon}, \xi_{2, \varepsilon}\right) \leq \mu_{2}$ one gets, with (3.1), the following estimate:

$$
\sum_{j=0}^{5}\left|\lambda_{2, j}^{\varepsilon}\right| \leq C \mu_{2}^{\frac{3}{2}}\left(\left\|\phi_{\varepsilon}\right\|_{C^{0}\left(2 r_{0}\right)}+\mu_{1}^{\frac{3}{2}}\right),
$$

so that, using (3.1) and Proposition 4.1, (5.26) improves into:

$$
\begin{aligned}
& \left|\phi_{\varepsilon}\left(x_{\varepsilon}\right)\right| \leq C\left(\nu_{\varepsilon}\left(\mu_{1}^{\frac{3}{2}}+\mu_{2}^{\frac{3}{2}}\right)+\mu_{1}^{\frac{7}{2}}+\mu_{2}^{\frac{3}{2}} \theta_{2}\left(x_{\varepsilon}\right)^{-1}+\mu_{1}^{\frac{3}{2}}\left(\frac{\mu_{2}}{\theta_{2}\left(x_{\varepsilon}\right)}\right)^{2}\right. \\
& \left.+\left(\mu_{1}^{2}+\left(\frac{\mu_{2}}{\theta_{2}\left(x_{\varepsilon}\right)}\right)^{2}\right)\left\|\phi_{\varepsilon}\right\|_{C^{0}\left(2 r_{0}\right)}\right) .
\end{aligned}
$$

As before, we prove the following claim:

Claim 5.4. There exists $0<\varepsilon_{2} \leq \varepsilon_{1}$ and $C>0$ such that, for any $0<\varepsilon \leq \varepsilon_{2}$, there holds:

$$
\left\|\phi_{\varepsilon}\right\|_{C^{0}\left(2 r_{0}\right)} \leq C \mu_{1}^{\frac{3}{2}}
$$

Proof. Here again we proceed by contradiction and assume that for some sequence $\left(\varepsilon_{k}\right)_{k}$ of positive numbers, $\varepsilon_{k} \rightarrow 0$ as $k \rightarrow+\infty$, there holds

$$
\left\|\phi_{k}\right\|_{C^{0}\left(2 r_{0}\right)} \gg \mu_{1}^{\frac{3}{2}} \text { as } k \rightarrow+\infty
$$

using the same notations as in the proof of Claim 5.3. Let $\left(y_{k}\right)_{k}$ be a sequence of points such that $\left|\phi_{k}\left(y_{k}\right)\right|=\left\|\phi_{k}\right\|_{C^{0}\left(2 r_{0}\right)}$. By (3.1), (3.4) and (5.30) there holds $\mu_{2}^{\frac{3}{2}} \theta_{2}\left(y_{k}\right)^{-1}=$ $o\left(\left\|\phi_{k}\right\|_{C^{0}\left(2 r_{0}\right)}\right)$ so that 5.28 and 5.30 show that

$$
\theta_{2}\left(y_{k}\right) \leq C \mu_{2} \text {. }
$$

For any $y \in B_{0}\left(\frac{r_{0}}{\mu_{2}}\right)$ we let again:

$$
\tilde{\phi}_{k}(y)=\frac{1}{\left\|\phi_{k}\right\|_{C^{0}\left(2 r_{0}\right)}} \phi_{k}\left(\exp _{\xi_{2}}\left(\mu_{2} y\right)\right) .
$$

Then (5.28) and (5.30) show that there holds:

$$
\left|\tilde{\phi}_{k}(y)\right| \leq C \frac{1}{(1+|y|)^{2}}+o(1) \text { for any } y \in B_{0}\left(\frac{r_{0}}{\mu_{2}}\right) \text {. }
$$


As before, 3.11), 3.12), Proposition 4.1, 5.25), (5.30) and standard elliptic theory show that $\tilde{\phi}_{k}$ converges, up to a subsequence, in $C_{l o c}^{1}\left(\mathbb{R}^{5}\right)$ towards $\tilde{\phi}_{0}$ as $k \rightarrow+\infty$, where $\tilde{\phi}_{0}$ solves in $\mathbb{R}^{5}$ :

$$
\triangle_{\text {eucl }} \tilde{\phi}_{0}=\frac{7}{3} U_{0}^{\frac{4}{3}} \tilde{\phi}_{0}+\sum_{j=0}^{5} \tilde{\lambda}_{2, j}^{0} V_{j},
$$

where eucl is the Euclidean metric, $U_{0}$ is as in (4.10), the $V_{j}$ are as in $(5.22)$ and for any $0 \leq j \leq 5$ we have let:

$$
\tilde{\lambda}_{2, j}^{0}=\lim _{k \rightarrow+\infty} \frac{\lambda_{2, j}^{\varepsilon_{k}}}{\mu_{2}^{\frac{3}{2}}\left\|\phi_{k}\right\|_{C^{0}\left(2 r_{0}\right)}},
$$

which exists, up to a subsequence, by (5.27) and (5.30). Passing (5.33) to the limit shows that $\tilde{\phi}_{0} \in L^{\frac{10}{3}}\left(\mathbb{R}^{5}\right)$ so that integrating (5.34) against $V_{j}$ shows first that $\tilde{\lambda}_{2, j}^{0}=0$ for any $0 \leq j \leq 5$ and then, by the result of [1], that:

$$
\tilde{\phi}_{0} \in \operatorname{Vect}\left\{V_{j}, 0 \leq j \leq 5\right\} \text {. }
$$

As before, we now prove that $\tilde{\phi}_{0} \in \operatorname{Vect}\left\{V_{j}, 0 \leq j \leq 5\right\}^{\perp}$. By Proposition 3.1 there holds again

$$
\left\langle\phi_{k}, Z_{j}\right\rangle=0
$$

for any $0 \leq j \leq 5$, where $\langle\cdot, \cdot\rangle$ is given by $(3.6)$, so that for any $R>0$ this implies that:

$$
\begin{aligned}
\int_{B_{g}\left(\xi_{2}, R \mu_{2}\right)}\left(\left\langle\nabla \phi_{k}, \nabla Z_{2, j}\right\rangle+\right. & \left.\left(\frac{3}{16} S_{g}+h\right) \phi_{k} Z_{2, j}\right) d v_{g}=\int_{\partial B_{g}\left(\xi_{2}, R \mu_{2}\right)} \partial_{\nu} Z_{2, j} \phi_{k} d \sigma_{g} \\
& -\int_{B_{g}\left(\xi_{2}, 2 r_{0}\right) \backslash B_{g}\left(\xi_{2}, R \mu_{2}\right)}\left(\triangle_{g}+\frac{3}{16} S_{g}+h\right) Z_{2, j} \phi_{k} d v_{g} .
\end{aligned}
$$

Straightforward computations using 2.10 show that there holds, for $y \in B_{\xi_{2}}\left(2 r_{0}\right)$ :

$$
\begin{aligned}
& \left|\triangle_{g} Z_{2,0}(y)-\frac{7}{3} W_{2}(y)^{\frac{4}{3}} Z_{2,0}(y)\right| \leq C \mu_{2}^{\frac{3}{2}} \theta_{2}(y)^{-3}, \\
& \left|\triangle_{g} Z_{2, j}(y)-\frac{7}{3} W_{2}(y)^{\frac{4}{3}} Z_{2, j}(y)\right| \leq C \mu_{2}^{\frac{5}{2}} \theta_{2}(y)^{-4}, \text { for } 1 \leq j \leq 5,
\end{aligned}
$$

where $\theta_{2}$ is as in (3.4). Now, 3.2, 5.28 and 5.30 show that:

$$
\left|\int_{\partial B_{g}\left(\xi_{2}, R \mu_{2}\right)} \partial_{\nu} Z_{2, j} \phi_{k} d \sigma_{g}\right| \leq\left(\frac{C}{1+R}+o(1)\right) \mu_{2}^{\frac{3}{2}}\left\|\phi_{k}\right\|_{C^{0}\left(2 r_{0}\right)},
$$

that

and that

$$
\left|\int_{B_{g}\left(\xi_{2}, 2 r_{0}\right) \backslash B_{g}\left(\xi_{2}, R \mu_{2}\right)} \mu_{2}^{\frac{3}{2}} \theta_{2}(\cdot)^{-3} \phi_{k} d v_{g}\right|=o\left(\mu_{2}^{\frac{3}{2}}\left\|\phi_{k}\right\|_{C^{0}\left(2 r_{0}\right)}\right),
$$

$$
\left|\int_{B_{g}\left(\xi_{2}, 2 r_{0}\right) \backslash B_{g}\left(\xi_{2}, R \mu_{2}\right)} W_{2}^{\frac{4}{3}} Z_{2, j} \phi_{k} d v_{g}\right| \leq\left(\frac{C}{(1+R)^{4}}+o(1)\right) \mu_{2}^{\frac{3}{2}}\left\|\phi_{k}\right\|_{C^{0}\left(2 r_{0}\right)},
$$


so that $(5.36)$ becomes:

$$
\left|\int_{B_{g}\left(\xi_{2}, R \mu_{2}\right)}\left(\left\langle\nabla \phi_{k}, \nabla Z_{2, j}\right\rangle+\left(\frac{3}{16} S_{g}+h\right) \phi_{k} Z_{2, j}\right) d v_{g}\right| \leq\left(\frac{C}{1+R}+o(1)\right) \mu_{2}^{\frac{3}{2}}\left\|\phi_{k}\right\|_{C^{0}\left(2 r_{0}\right)} .
$$

Dividing both sides by $\mu_{2}^{\frac{3}{2}}\left\|\phi_{k}\right\|_{C^{0}\left(2 r_{0}\right)}$, letting first the $k \rightarrow+\infty$ and then $R \rightarrow+\infty$ gives as before that $\tilde{\phi}_{0} \in \operatorname{Vect}\left\{V_{j}, 0 \leq j \leq 5\right\}^{\perp}$ and hence $\tilde{\phi}_{0} \equiv 0$. But this is a contradiction with (5.35), since $\left|\tilde{\phi}_{0}\left(\tilde{y}_{0}\right)\right|=1$ by (5.31), where $\tilde{y}_{0}$ is the limit of $\frac{1}{\mu_{2}} \exp _{\xi_{2}}^{-1}\left(y_{k}\right)$, and concludes the proof of Claim 5.4 .

Now, plugging (5.29) into $(5.28)$ yields:

$$
\left|\phi_{\varepsilon}\left(x_{\varepsilon}\right)\right| \leq C\left(\nu_{\varepsilon}\left(\mu_{1}^{\frac{3}{2}}+\mu_{2}^{\frac{3}{2}}\right)+\mu_{1}^{\frac{7}{2}}+\mu_{2}^{\frac{3}{2}} \theta_{2}\left(x_{\varepsilon}\right)^{-1}+\mu_{1}^{\frac{3}{2}}\left(\frac{\mu_{2}}{\theta_{2}\left(x_{\varepsilon}\right)}\right)^{2}\right) .
$$

Writing down again a representation formula for (5.25) and using the latter to estimate the term involving $\phi_{\varepsilon}$ then concludes the proof of Lemma 5.2 for $n=5$.

Note that the precision that we reach in Lemma 5.2 is related to the nature of $\phi_{\varepsilon}$, in particular to the property of $\phi_{\varepsilon}$ to be orthogonal to the kernel elements.

End of the proof of Proposition 5.1. Of course the constants $\varepsilon_{2}$ and $C$ given by Lemma 5.2 do depend on the choice of the family $\left(t_{1, \varepsilon}, \xi_{1, \varepsilon}, t_{2, \varepsilon}, \xi_{2, \varepsilon}\right)_{\varepsilon \leq \varepsilon_{1}}$. To conclude the proof of Proposition 5.1 we establish as before their uniformity. We only write the argument for $n=4$ since the $n=5$ case works identically. First, the right-hand side of (5.2) (seen as a continuous function in $M$ ) is obviously continuous in $\left(t_{1}, \xi_{1}, t_{2}, \xi_{2}\right)$. Therefore, by Lemma 4.2, there exists a family $\left(t_{1, \varepsilon}, \xi_{1, \varepsilon}, t_{2, \varepsilon}, \xi_{2, \varepsilon}\right)_{0<\varepsilon \leq \varepsilon_{1}} \in A$ such that for any $0<\varepsilon \leq \varepsilon_{1}$ there holds:

$$
\begin{aligned}
& \left.\left\|\frac{\phi_{\varepsilon}\left(t_{1, \varepsilon}, \xi_{1, \varepsilon}, t_{2, \varepsilon}, \xi_{2, \varepsilon}\right)}{\| \nu_{\varepsilon}\left(\mu_{1, \varepsilon}+\mu_{2, \varepsilon}\right)+\mu_{2, \varepsilon}\left|\ln \theta_{2}(\cdot)\right|+\mu_{1, \varepsilon} \mu_{2, \varepsilon}\left|\ln \left(\frac{\theta_{2, \varepsilon}(\cdot)}{\mu_{2}}\right)\right| \frac{\mu_{2}}{\theta_{2}(\cdot)^{2}}}\right\|\right|_{C^{0}\left(\overline{B_{g}\left(\xi_{2,0}, r_{0}\right)}\right)} \\
& =\left.\sup _{\left(t_{1}, \xi_{1}, t_{2}, \xi_{2}\right) \in A}\left\|\frac{\phi_{\varepsilon}\left(t_{1}, \xi_{1}, t_{2}, \xi_{2}\right)}{\nu_{\varepsilon}\left(\mu_{1}+\mu_{2}\right)+\mu_{2}\left|\ln \theta_{2}(\cdot)\right|+\mu_{1} \mu_{2}\left|\ln \left(\frac{\theta_{2}(\cdot)}{\mu_{2}}\right)\right| \frac{\mu_{2}}{\theta_{2}(\cdot)^{2}}}\right\|\right|_{C^{0}\left(\overline{B_{g}\left(\xi_{2,0}, r_{0}\right)}\right)},
\end{aligned}
$$

where $\xi_{2,0}$ and $r_{0}$ are as in (2.6), $\nu_{\varepsilon}$ is given by Proposition 4.1 and $A$ is given by (3.3). Note in particular that $\xi_{2, \varepsilon} \in \overline{B_{g}\left(\xi_{2,0}, r_{0}\right)}$. Let $\varepsilon_{2}$ and $C$ be the constants associated to this family $\left(t_{1, \varepsilon}, \xi_{1, \varepsilon}, t_{2, \varepsilon}, \xi_{2, \varepsilon}\right)_{0<\varepsilon \leq \varepsilon_{1}}$ by Lemma 5.2 . Let now $\xi_{2} \in \overline{B_{g}\left(\xi_{2,0}, \frac{r_{0}}{2}\right)}$. Then:

$$
\overline{B_{g}\left(\xi_{2}, \frac{r_{0}}{2}\right)} \subset \overline{B_{g}\left(\xi_{2,0}, r_{0}\right)}
$$


Therefore, for any $0<\varepsilon \leq \varepsilon_{2}$,

$$
\begin{aligned}
& \sup _{\left(t_{1}, \xi_{1}, t_{2}, \xi_{2}\right) \in A_{1}}\left\|\frac{\phi_{\varepsilon}\left(t_{1}, \xi_{1}, t_{2}, \xi_{2}\right)}{\nu_{\varepsilon}\left(\mu_{1}+\mu_{2}\right)+\mu_{2}\left|\ln \theta_{2}(\cdot)\right|+\mu_{1} \mu_{2}\left|\ln \left(\frac{\theta_{2}(\cdot)}{\mu_{2}}\right)\right| \frac{\mu_{2}}{\theta_{2}(\cdot)^{2}}}\right\|_{C^{0}\left(\frac{B_{g}\left(\xi_{2}, \frac{r_{0}}{2}\right)}{2}\right)} \| \phi_{\varepsilon}\left(t_{1}, \xi_{1}, t_{2}, \xi_{2}\right) \\
& \leq \sup _{\left(t_{1}, \xi_{1}, t_{2}, \xi_{2}\right) \in A_{1}} \| \frac{\mu_{1}\left|\ln \left(\frac{\theta_{2}(\cdot)}{\mu_{2}}\right)\right| \frac{\mu_{2}}{\theta_{2}(\cdot)^{2}}}{\nu_{\varepsilon}\left(\mu_{1}+\mu_{2}\right)+\mu_{2}\left|\ln \theta_{2}(\cdot)+\mu_{1} \mu_{2}\right| \ln \left(\overline{B_{g}\left(\xi_{2,0}, r_{0}\right)}\right)} \\
& \leq C,
\end{aligned}
$$

where in the last inequality we used that $A_{1} \subset A$ and $\overline{B_{g}\left(\xi_{2,0}, r_{0}\right)} \subset \overline{B_{g}\left(\xi_{2, \varepsilon}, 2 r_{0}\right)}$ in order to apply (5.38) and Lemma 5.2. This concludes the proof of (5.2).

\section{Asymptotic Expansion Along the KeRnel}

For any $0<\varepsilon \leq \varepsilon_{2}$ and $\left(t_{1}, \xi_{1}, t_{2}, \xi_{2}\right) \in A_{1}$ we let $\phi_{\varepsilon}=\phi_{\varepsilon}\left(t_{1}, \xi_{1}, t_{2}, \xi_{2}\right)$ be given by Proposition 3.1, where $A_{1}$ is as in (5.1) and $\varepsilon_{2}$ is given by Proposition 5.1. In this Section we obtain an asymptotic expansion of the functions $\lambda_{i, j}^{\varepsilon}, 1 \leq i \leq 2,0 \leq j \leq n$ defined in (4.19). Throughout this section, all the asymptotic expansions that we will write hold in $C^{0}\left(A_{1}\right)$.

6.1. Expansion of the $\lambda_{1, j}^{\varepsilon}, 0 \leq j \leq n$. We first obtain an asymptotic expansion of the $\lambda_{1, j}^{\varepsilon}, 0 \leq j \leq n$ :

Lemma 6.1. The following expansions hold in $C^{0}\left(A_{1}\right)$ as $\varepsilon \rightarrow 0$, where $A_{1}$ is as in (5.1):

If $n=4$ :

$$
\begin{aligned}
& \left\|\nabla V_{0}\right\|_{L^{2}\left(\mathbb{R}^{4}\right)}^{2} \lambda_{1,0}^{\varepsilon}\left(t_{1}, \xi_{1}, t_{2}, \xi_{2}\right)= \\
& 2\left(C_{1}(4) H\left(\xi_{1}\right) t_{1}-C_{2}(4) A\left(\xi_{1}\right)+\frac{C_{3}(4)}{2} F_{h}\left(\xi_{1}\right)\right) e^{-\frac{2 t_{1}}{\varepsilon}}+o\left(e^{-\frac{2 t_{1}}{\varepsilon}}\right), \\
& \left\|\nabla V_{j}\right\|_{L^{2}\left(\mathbb{R}^{4}\right)}^{2} \lambda_{1, j}^{\varepsilon}\left(t_{1}, \xi_{1}, t_{2}, \xi_{2}\right)= \\
& 4\left(C_{1}(4) \nabla_{j} H\left(\xi_{1}\right) t_{1}-C_{2}(4) \nabla_{j} A\left(\xi_{1}\right)+C_{3}(4)\left(\nabla_{j} F_{h}\right)\left(\xi_{1}\right)\right) e^{-\frac{3 t_{1}}{\varepsilon}}+o\left(e^{-\frac{3 t_{1}}{\varepsilon}}\right) .
\end{aligned}
$$

If $n=5$ :

$$
\begin{aligned}
& \left\|\nabla V_{0}\right\|_{L^{2}\left(\mathbb{R}^{5}\right)}^{2} \lambda_{1,0}^{\varepsilon}\left(t_{1}, \xi_{1}, t_{2}, \xi_{2}\right)= \\
& \left(\frac{4}{3} C_{1}(5) H\left(\xi_{1}\right) t_{1}^{2}-2 C_{2}(5) t_{1}^{3} A\left(\xi_{1}\right)+2 C_{3}(5) t_{1}^{3} F_{h}\left(\xi_{1}\right)\right) \varepsilon^{3}+o\left(\varepsilon^{3}\right), \\
& \left\|\nabla V_{j}\right\|_{L^{2}\left(\mathbb{R}^{5}\right)}^{2} \lambda_{1, j}^{\varepsilon}\left(t_{1}, \xi_{1}, t_{2}, \xi_{2}\right)= \\
& 5\left(C_{1}(5) \nabla_{j} H\left(\xi_{1}\right) t_{1}^{3}-C_{2}(5) \nabla_{j} A\left(\xi_{1}\right) t_{1}^{4}+C_{3}(5)\left(\nabla_{j} F_{h}\right)\left(\xi_{1}\right) t_{1}^{4}\right) \varepsilon^{4}+o\left(\varepsilon^{4}\right) .
\end{aligned}
$$

In (6.1) and (6.2) the $V_{j}$ are defined in (5.22), A( $\left.\xi_{1}\right)$ denotes the mass of the Green's function of the operator $G_{\xi_{1}}$ at $\xi_{1}$ defined in (2.4), $F_{h}$ is defined in $B_{g_{\xi_{1,0}}}\left(\xi_{1,0}, 2 r_{0}\right)$ and 
given by

$$
F_{h}(\xi)=\int_{M} G_{h}(\xi, y) h(y) G_{g}\left(y, \xi_{1}\right) d v_{g}(y),
$$

$C_{1}(n), C_{2}(n)$ are positive constants given by 6.13 , and $C_{3}(n)$ is a positive constant defined by 6.10 below.

The explicit values of the constants $C_{i}(n)$ do not come into play in our final argument. It is important to notice that the term $A\left(\xi_{1}\right)-F_{h}\left(\xi_{1}\right)$ is just, in view of $(2.12)$, the mass of the Green's function $G_{h}\left(\xi_{1}, \cdot\right)$ at $\xi_{1}$ by analogy with (2.4). Note that the mass of $G_{h}\left(\xi_{1}, \cdot\right)$ at $\xi_{1}$ exists because $h$ is supported in $M \backslash B_{g_{\xi_{1}, 0}}\left(\xi_{1,0}, 2 r_{0}\right)$. If $h$ were just a smooth function in $M$ the next order term in expansion 2.4 would likely be singular too, as explained in [24].

Proof. These expansions only require the $H^{1}$ estimate on $\phi_{\varepsilon}$ given by (3.8). By (3.2), the $Z_{1, j}, 0 \leq j \leq n$ are supported in $B_{g_{\xi_{1,0}}}\left(\xi_{1,0}, 2 r_{0}\right)$. Since $W_{2}$, the $Z_{2, j}$ and $h$ are supported in $M \backslash B_{g_{\xi_{1,0}}}\left(\xi_{1,0}, 2 r_{0}\right)$ it is easily seen that 4.19 rewrites in $B_{g_{\xi_{1,0}}}\left(\xi_{1,0}, 2 r_{0}\right)$ as:

$$
\begin{aligned}
\sum_{j=0}^{n} \lambda_{1, j}^{\varepsilon}\left(t_{1}, \xi_{1}, t_{2}, \xi_{2}\right) & \left(\triangle_{g}+c_{n} S_{g}+\varepsilon H\right) Z_{1, j}=\varepsilon H T_{1} \\
& +\left(\triangle_{g}+c_{n} S_{g}+\varepsilon H\right)\left(\hat{W}_{1}+\phi_{\varepsilon}\right)-\left(\hat{W}_{1}+\phi_{\varepsilon}\right)^{2^{*}-1} \\
& -\left[\left(\hat{W}_{1}+T_{1}+\phi_{\varepsilon}\right)^{2^{*}-1}-\left(\hat{W}_{1}+\phi_{\varepsilon}\right)^{2^{*}-1}\right],
\end{aligned}
$$

where $T_{1}$ is given by (2.8) with $\mu_{1}$ given by (3.1). To estimate the $\lambda_{1, j}^{\varepsilon}$ we integrate 6.4 against $Z_{1, j}$. First, by (2.11), (3.1) and (3.2) there holds, for $0 \leq j \leq n$ :

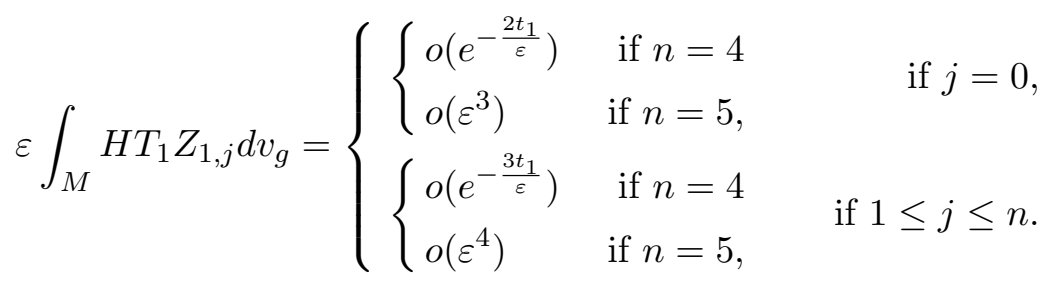

Then, we write that

$$
\begin{aligned}
&\left|\left(\hat{W}_{1}+T_{1}+\phi_{\varepsilon}\right)^{2^{*}-1}-\left(\hat{W}_{1}+\phi_{\varepsilon}\right)^{2^{*}-1}-\left(2^{*}-1\right) \hat{W}_{1}^{2^{*}-2} T_{1}\right| \\
& \lesssim\left(\hat{W}_{1}+\phi_{\varepsilon}\right)^{2^{*}-3}\left|T_{1}\right|^{2}+\left|T_{1}\right|^{2^{*}-1}+\left|\left(\hat{W}_{1}+\phi_{\varepsilon}\right)^{2^{*}-2}-\hat{W}_{1}^{2^{*}-2}\right|\left|T_{1}\right| \\
& \lesssim\left(\left|\phi_{\varepsilon}\right|^{2^{*}-2}+\hat{W}_{1}^{2^{*}-3}\left|\phi_{\varepsilon}\right|\right)\left|T_{1}\right|+\left(\hat{W}_{1}^{2^{*}-3}+\left|\phi_{\varepsilon}\right|^{2^{*}-3}\right)\left|T_{1}\right|^{2}+\left|T_{1}\right|^{2^{*}-1}
\end{aligned}
$$

Straightforward computations with (2.11), 3.1) and (3.2) give that:

$$
\int_{M}\left(\hat{W}_{1}^{2^{*}-3}\left|T_{1}\right|^{2}+\left|T_{1}\right|^{2^{*}-1}\right)\left|Z_{1, j}\right| d v_{g}=\left\{\begin{array}{l}
\left\{\begin{array}{ll}
o\left(e^{\left.-\frac{2 t_{1}}{\varepsilon}\right)}\right. & \text { if } n=4 \\
o\left(\varepsilon^{3}\right) & \text { if } n=5,
\end{array} \quad \text { if } j=0,\right. \\
\left\{\begin{array}{ll}
o\left(e^{\left.-\frac{3 t_{1}}{\varepsilon}\right)}\right. & \text { if } n=4 \\
o\left(\varepsilon^{4}\right) & \text { if } n=5,
\end{array} \quad \text { if } 1 \leq j \leq n,\right.
\end{array}\right.
$$


while (2.11), (3.1), (3.2) and (3.8) give, with Hölder's inequality:

$$
\begin{array}{r}
\int_{M}\left(\left|\phi_{\varepsilon}\right|^{2^{*}-2}\left|T_{1}\right|+\hat{W}_{1}^{2^{*}-3}\left|\phi_{\varepsilon}\right|\left|T_{1}\right|+\left|\phi_{\varepsilon}\right|^{2^{*}-3}\left|T_{1}\right|^{2}\right)\left|Z_{1, j}\right| d v_{g}= \\
\begin{cases}\left\{\begin{array}{ll}
o\left(e^{-\frac{2 t_{1}}{\varepsilon}}\right) & \text { if } n=4 \\
o\left(\varepsilon^{3}\right) & \text { if } n=5, \\
\left\{\left(e^{-\frac{3 t_{1}}{\varepsilon}}\right)\right. & \text { if } n=4 \\
o\left(\varepsilon^{4}\right) & \text { if } n=5,
\end{array} \quad \text { if } j=0,\right.\end{cases}
\end{array}
$$

Combining the latter computations in 6.6 then gives:

$$
\begin{aligned}
& -\int_{M}\left[\left(\hat{W}_{1}+T_{1}+\phi_{\varepsilon}\right)^{2^{*}-1}-\left(\hat{W}_{1}+\phi_{\varepsilon}\right)^{2^{*}-1}\right] Z_{1, j} d v_{g}=
\end{aligned}
$$

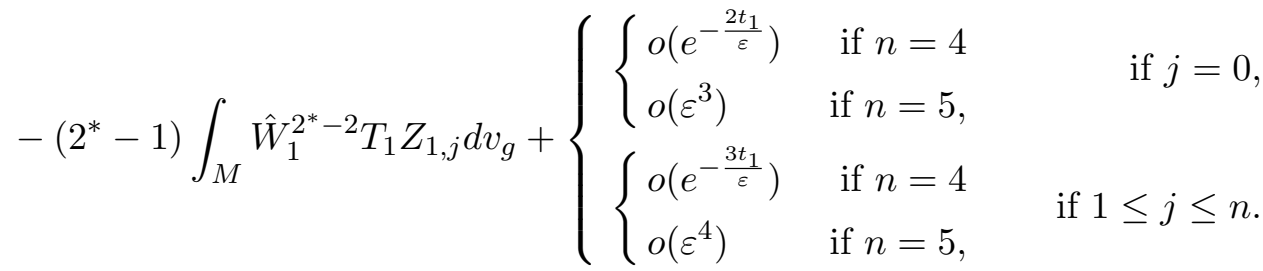

With (2.11) and $(3.2)$ it is now easily seen that there holds:

$$
-\left(2^{*}-1\right) \int_{M} \hat{W}_{1}^{2^{*}-2} T_{1} Z_{1,0} d v_{g}=C_{3}(n) \mu_{1}^{n-2} F_{h}\left(\xi_{1}\right)+o\left(\mu_{1}^{n-2}\right)
$$

and

$$
-\left(2^{*}-1\right) \int_{M} \hat{W}_{1}^{2^{*}-2} T_{1} Z_{1, j} d v_{g}=n C_{3}(n) \mu_{1}^{n-1}\left(\nabla_{j} F_{h}\right)\left(\xi_{1}\right)+o\left(\mu_{1}^{n-1}\right),
$$

where $\mu_{1}$ is given by $(3.1)$ and where $C_{3}(n)>0$ is given by the following expansion as $\varepsilon \rightarrow 0$ :

$$
-\int_{M} \hat{W}_{1}^{2^{*}-1} T_{1} d v_{g}=C_{3}(n) \mu_{1}^{n-2} F_{h}\left(\xi_{1}\right)+O\left(\mu_{1}^{n}\right),
$$

for $F_{h}$ defined in (6.3). Finally, by (2.7), (3.1), (3.8) and since by construction $\phi_{\varepsilon}$ is orthogonal to the $Z_{1, j}, 0 \leq j \leq n$, an adaptation of the computations in [8] (Section 6) shows that there holds:

$$
\begin{aligned}
\int_{M}\left[\left(\triangle_{g}+c_{n} S_{g}+\varepsilon H\right)\left(\hat{W}_{1}+\phi_{\varepsilon}\right)-\left(\hat{W}_{1}+\phi_{\varepsilon}\right)^{2^{*}-1}\right] Z_{1,0} d v_{g}= & \\
2\left(C_{1}(4) H\left(\xi_{1}\right) t_{1}-C_{2}(4) A\left(\xi_{1}\right)\right) e^{-\frac{2 t_{1}}{\varepsilon}}+o\left(e^{-\frac{2 t_{1}}{\varepsilon}}\right) & \text { if } n=4, \\
& \left(\frac{4}{3} C_{1}(5) H\left(\xi_{1}\right) t_{1}^{2}-2 C_{2}(5) t_{1}^{3} A\left(\xi_{1}\right)\right) \varepsilon^{3}+o\left(\varepsilon^{3}\right) \quad \text { if } n=5
\end{aligned}
$$


and

$$
\begin{array}{rlr}
\int_{M}\left[\left(\triangle_{g}+c_{n} S_{g}+\varepsilon H\right)\left(\hat{W}_{1}+\phi_{\varepsilon}\right)-\left(\hat{W}_{1}+\phi_{\varepsilon}\right)^{2^{*}-1}\right] Z_{1, j} d v_{g}= & \\
& 4\left(C_{1}(4) \nabla_{j} H\left(\xi_{1}\right) t_{1}-C_{2}(4) \nabla_{j} A\left(\xi_{1}\right)\right) e^{-\frac{3 t_{1}}{\varepsilon}}+o\left(e^{-\frac{3 t_{1}}{\varepsilon}}\right) & \text { if } n=4, \\
5\left(C_{1}(5) \nabla_{j} H\left(\xi_{1}\right) t_{1}^{3}-C_{2}(5) \nabla_{j} A\left(\xi_{1}\right) t_{1}^{4}\right) \varepsilon^{4}+o\left(\varepsilon^{4}\right) & \text { if } n=5,
\end{array}
$$

where $C_{1}(n)$ and $C_{2}(n)$ are two positive constants defined by the following expansion as $\varepsilon \rightarrow 0$ :

$$
\begin{aligned}
& \frac{1}{2} \int_{M}\left|\nabla \hat{W}_{1}\right|_{g}^{2}+\left(c_{n} S_{g}+\varepsilon H\right) \hat{W}_{1}^{2} d v_{g}-\frac{1}{2^{*}} \int_{M} \hat{W}_{1}^{2^{*}} d v_{g} \\
& \quad=\frac{1}{n} K_{n}^{-n}+ \begin{cases}\left(C_{1}(4) H\left(\xi_{1}\right) t_{1}-C_{2}(4) A\left(\xi_{1}\right)\right) e^{-\frac{2 t_{1}}{\varepsilon}}+o\left(e^{-\frac{2 t_{1}}{\varepsilon}}\right) & \text { if } n=4 \\
\left(C_{1}(5) H\left(\xi_{1}\right) t_{1}^{2}-C_{2}(5) A\left(\xi_{1}\right) t_{1}^{3}\right) \varepsilon^{3}+o\left(\varepsilon^{3}\right) & \text { if } n=5 .\end{cases}
\end{aligned}
$$

It remains to notice that by $(3.2)$ there holds:

$$
\left\langle Z_{1, j}, Z_{1, k}\right\rangle=\delta_{j k}\left\|\nabla V_{j}\right\|_{L^{2}\left(\mathbb{R}^{n}\right)}^{2}+o(1)
$$

for $0 \leq j, k \leq n$, and even:

$$
\left\langle Z_{1,0}, Z_{1, j}\right\rangle= \begin{cases}o\left(e^{-\frac{t_{1}}{\varepsilon}}\right) & \text { if } n=4 \\ o(\varepsilon) & \text { if } n=5\end{cases}
$$

for $1 \leq j \leq n$, in $C^{0}\left(A_{1}\right)$ as $\varepsilon \rightarrow 0$, where $\langle\cdot, \cdot\rangle$ is given by $(3.6)$ and $V_{j}$ is as in 5.22 . With (3.1), (6.4), (6.5), (6.7), (6.8), (6.9), (6.11) and (6.12) this concludes the proof of the Lemma.

6.2. Expansion of the $\lambda_{2, j}^{\varepsilon}, 0 \leq j \leq n$. In this subsection we obtain an asymptotic expansion of the $\lambda_{2, j}^{\varepsilon}$. Unlike the case of the $\lambda_{1, j}^{\varepsilon}$, the expansion of the $\lambda_{2, j}^{\varepsilon}$ now crucially relies on the precise pointwise asymptotics on $\phi_{\varepsilon}$ obtained in Sections 4 and 5 .

Lemma 6.2. The following expansions hold in $C^{0}\left(A_{1}\right)$ as $\varepsilon \rightarrow 0$, where $A_{1}$ is as in (5.1): If $n=4$ :

$$
\begin{aligned}
& \left\|\nabla V_{0}\right\|_{L^{2}\left(\mathbb{R}^{4}\right)}^{2} \lambda_{2,0}^{\varepsilon}\left(t_{1}, \xi_{1}, t_{2}, \xi_{2}\right)= \\
& \left(2 D_{1}(4) h\left(\xi_{2}\right) t_{1} t_{2}^{2}-D_{2}(4) t_{2} G_{h}\left(\xi_{1}, \xi_{2}\right)\right) \varepsilon e^{-\frac{2 t_{1}}{\varepsilon}}+o\left(\varepsilon e^{-\frac{2 t_{1}}{\varepsilon}}\right) \\
& \left\|\nabla V_{j}\right\|_{L^{2}\left(\mathbb{R}^{4}\right)}^{2} \lambda_{2, j}^{\varepsilon}\left(t_{1}, \xi_{1}, t_{2}, \xi_{2}\right)= \\
& 4\left(D_{1}(4) \nabla_{j} h\left(\xi_{2}\right) t_{1} t_{2}^{3}-D_{2}(4) t_{2}^{2} \nabla_{j} G_{h}\left(\xi_{1}, \xi_{2}\right)\right) \varepsilon^{2} e^{-\frac{3 t_{1}}{\varepsilon}}+o\left(\varepsilon^{2} e^{-\frac{3 t_{1}}{\varepsilon}}\right) .
\end{aligned}
$$


If $n=5:$

$$
\begin{aligned}
& \left\|\nabla V_{0}\right\|_{L^{2}\left(\mathbb{R}^{5}\right)}^{2} \lambda_{2,0}^{\varepsilon}\left(t_{1}, \xi_{1}, t_{2}, \xi_{2}\right)= \\
& \left(\frac{4}{3} D_{1}(5) h\left(\xi_{2}\right) t_{2}^{2}-D_{2}(5)\left(t_{1} t_{2}\right)^{\frac{3}{2}} G_{h}\left(\xi_{1}, \xi_{2}\right)\right) \varepsilon^{6}+o\left(\varepsilon^{6}\right), \\
& \left\|\nabla V_{j}\right\|_{L^{2}\left(\mathbb{R}^{5}\right)}^{2} \lambda_{2, j}^{\varepsilon}\left(t_{1}, \xi_{1}, t_{2}, \xi_{2}\right)= \\
& 5\left(D_{1}(5) \nabla_{j} h\left(\xi_{2}\right) t_{2}^{3}-D_{2}(5) t_{1}^{\frac{3}{2}} t_{2}^{\frac{5}{2}} \nabla_{j} G_{h}\left(\xi_{1}, \xi_{2}\right)\right) \varepsilon^{9}+o\left(\varepsilon^{9}\right) .
\end{aligned}
$$

In (6.14) and (6.15) the $V_{j}$ are as in (5.22), $G_{h}$ denotes the Green's function of $\triangle_{g}+$ $c_{n} S_{g}+h$ in $M$ and its derivative is taken with respect to $\xi_{2}$. Also, $D_{1}(n)$ and $D_{2}(n)$ are positive constants given by (6.20) and (6.23) below.

Proof. As before, all the asymptotic expansions that we will write here take place in $C^{0}\left(A_{1}\right)$. By (2.7), 2.8), (3.2) and (4.19) and since the $Z_{1, j}$ and $H$ vanish on $B_{g}\left(\xi_{2}, 2 r_{0}\right)$, for any $0<\varepsilon \leq \varepsilon_{2}$ and for any $\left(t_{1}, \xi_{1}, t_{2}, \xi_{2}\right) \in A_{1}$ there holds:

$$
\begin{aligned}
\sum_{j=0}^{n} & \lambda_{2, j}^{\varepsilon}\left(t_{1}, \xi_{1}, t_{2}, \xi_{2}\right)\left(\triangle_{g}+c_{n} S_{g}+h\right) Z_{2, j}=\left(\triangle_{g}+c_{n} S_{g}\right) \hat{W}_{1}-\hat{W}_{1}^{2^{*}-1} \\
& +\hat{W}_{1}^{2^{*}-1}-\left(\hat{W}_{1}+T_{1}\right)^{2^{*}-1} \\
& +\left(\triangle_{g}+c_{n} S_{g}+h\right) W_{2}-W_{2}^{2^{*}-1} \\
& -\left(2^{*}-1\right) W_{2}^{2^{*}-2} W_{1} \\
& -\left[\left(W_{1}+W_{2}\right)^{2^{*}-1}-W_{1}^{2^{*}-1}-W_{2}^{2^{*}-1}-\left(2^{*}-1\right) W_{2}^{2^{*}-2} W_{1}\right] \\
& -\left[\left(W_{1}+W_{2}+\phi_{\varepsilon}\right)^{2^{*}-1}-\left(W_{1}+W_{2}\right)^{2^{*}-1}-\left(2^{*}-1\right)\left(W_{1}+W_{2}\right)^{2^{*}-2} \phi_{\varepsilon}\right] \\
& +\left(\triangle_{g}+c_{n} S_{g}+h\right) \phi_{\varepsilon}-\left(2^{*}-1\right) W_{2}^{2^{*}-2} \phi_{\varepsilon} \\
& -\left(2^{*}-1\right)\left[\left(W_{1}+W_{2}\right)^{2^{*}-2}-W_{2}^{2^{*}-2}\right] \phi_{\varepsilon} .
\end{aligned}
$$

We integrate (6.16) against $Z_{2, j}$ for $0 \leq j \leq n$. First, using (2.7), (2.11), (3.2) and (3.11) we get that, for any $0 \leq j \leq n$ :

$$
\begin{array}{r}
\int_{M}\left[\left(\triangle_{g}+c_{n} S_{g}\right) \hat{W}_{1}-\hat{W}_{1}^{2^{*}-1}+\hat{W}_{1}^{2^{*}-1}-\left(\hat{W}_{1}+T_{1}\right)^{2^{*}-1}\right] Z_{2, j} d v_{g} \\
= \begin{cases} \begin{cases}o\left(\varepsilon e^{-\frac{2 t_{1}}{\varepsilon}}\right) & \text { if } n=4 \\
o\left(\varepsilon^{6}\right) & \text { if } n=5,\end{cases} & \text { if } j=0, \\
\begin{cases}o\left(\varepsilon^{2} e^{-\frac{3 t_{1}}{\varepsilon}}\right) & \text { if } n=4 \\
o\left(\varepsilon^{9}\right) & \text { if } n=5,\end{cases} & \text { if } 1 \leq j \leq n .\end{cases}
\end{array}
$$


Mimicking the computations that led to 6.8 and $(6.9)$ we get that:

$$
\begin{aligned}
\int_{M}\left[\left(\triangle_{g}+c_{n} S_{g}+h\right) W_{2}-W_{2}^{2^{*}-1}\right] Z_{2,0} d v_{g}= \\
\left\{\begin{array}{ll}
2 D_{1}(4) h\left(\xi_{2}\right) \varepsilon t_{1} t_{2}^{2} e^{-\frac{2 t_{1}}{\varepsilon}}+o\left(\varepsilon e^{-\frac{2 t_{1}}{\varepsilon}}\right) & \text { if } n=4 \\
\frac{4}{3} D_{1}(5) h\left(\xi_{2}\right) \varepsilon^{6} t_{2}^{2}+o\left(\varepsilon^{6}\right) & \text { if } n=5
\end{array},\right.
\end{aligned}
$$

and

$$
\begin{gathered}
\int_{M}\left[\left(\triangle_{g}+c_{n} S_{g}+h\right) W_{2}-W_{2}^{2^{*}-1}\right] Z_{2, j} d v_{g}= \\
\left\{\begin{array}{ll}
4 D_{1}(4) \nabla_{j} h\left(\xi_{2}\right) \varepsilon^{2} t_{1} t_{2}^{3} e^{-\frac{3 t_{1}}{\varepsilon}}+o\left(\varepsilon^{2} e^{-\frac{3 t_{1}}{\varepsilon}}\right) & \text { if } n=4 \\
5 D_{1}(5) \nabla_{j} h\left(\xi_{2}\right) \varepsilon^{9} t_{2}^{3}+o\left(\varepsilon^{9}\right) & \text { if } n=5
\end{array},\right.
\end{gathered}
$$

where the positive constants $D_{1}(4)$ and $D_{1}(5)$ are defined by:

$$
\begin{aligned}
\frac{1}{2} \int_{M}\left|\nabla W_{2}\right|_{g}^{2} & +\left(c_{n} S_{g}+h\right) W_{2}^{2} d v_{g}-\frac{1}{2^{*}} \int_{M} W_{2}^{2^{*}} d v_{g} \\
& =\frac{1}{n} K_{n}^{-n}+ \begin{cases}D_{1}(4) h\left(\xi_{2}\right) \varepsilon t_{1} t_{2}^{2} e^{-\frac{2 t_{1}}{\varepsilon}}+o\left(\varepsilon e^{-\frac{2 t_{1}}{\varepsilon}}\right) & \text { if } n=4 \\
D_{1}(5) h\left(\xi_{2}\right) \varepsilon^{6} t_{2}^{2}+o\left(\varepsilon^{6}\right) & \text { if } n=5,\end{cases}
\end{aligned}
$$

where $K_{n}$ is defined in (4.6). Similarly, direct computations using (2.13), (3.1) and (3.2) show that

$$
-\left(2^{*}-1\right) \int_{M} W_{2}^{2^{*}-2} W_{1} Z_{2,0} d v_{g}= \begin{cases}-D_{2}(4) \varepsilon t_{2} e^{-\frac{2 t_{1}}{\varepsilon}} G_{h}\left(\xi_{1}, \xi_{2}\right)+o\left(\varepsilon e^{-\frac{2 t_{1}}{\varepsilon}}\right) & \text { if } n=4, \\ -D_{2}(5) \varepsilon^{6}\left(t_{1} t_{2}\right)^{\frac{3}{2}} G_{h}\left(\xi_{1}, \xi_{2}\right)+o\left(\varepsilon^{6}\right) & \text { if } n=5,\end{cases}
$$

and, for $0 \leq j \leq n$, that

$$
-\left(2^{*}-1\right) \int_{M} W_{2}^{2^{*}-2} W_{1} Z_{2, j} d v_{g}= \begin{cases}-4 D_{2}(4) \varepsilon^{2} t_{2}^{2} e^{-\frac{3 t_{1}}{\varepsilon}} \nabla_{j} G_{h}\left(\xi_{1}, \xi_{2}\right)+o\left(\varepsilon^{2} e^{-\frac{3 t_{1}}{\varepsilon}}\right) & \text { if } n=4, \\ -5 D_{2}(5) \varepsilon^{9} t_{1}^{\frac{3}{2}} t_{2}^{\frac{5}{2}} \nabla_{j} G_{h}\left(\xi_{1}, \xi_{2}\right)+o\left(\varepsilon^{9}\right) & \text { if } n=5,\end{cases}
$$

where $\nabla G\left(\xi_{1}, \xi_{2}\right)$ stands for the derivative of $G_{h}\left(\xi_{1}, \cdot\right)$ at $\xi_{2}$. Also, in 6.21 and 6.22, the positive constants $D_{2}(n)$ are given by the following expansion as $\varepsilon \rightarrow 0$ :

$$
\int_{M} W_{2}^{2^{*}-1} W_{1} d v_{g}= \begin{cases}D_{2}(4) \varepsilon t_{2} e^{-\frac{2 t_{1}}{\varepsilon}} G_{h}\left(\xi_{1}, \xi_{2}\right)+o\left(\varepsilon e^{-\frac{2 t_{1}}{\varepsilon}}\right) & \text { if } n=4 \\ D_{2}(5) \varepsilon^{6}\left(t_{1} t_{2}\right)^{\frac{3}{2}} G_{h}\left(\xi_{1}, \xi_{2}\right)+o\left(\varepsilon^{6}\right) & \text { if } n=5 .\end{cases}
$$

Finally, writing that

$$
\left|\left(W_{1}+W_{2}\right)^{2^{*}-1}-W_{1}^{2^{*}-1}-W_{2}^{2^{*}-1}-\left(2^{*}-1\right) W_{2}^{2^{*}-2} W_{1}\right| \lesssim W_{2}^{2^{*}-3} W_{1}^{2}+W_{1}^{2^{*}-1}
$$


and using 2.13 and 3.2 yields:

$$
\begin{aligned}
& \int_{M}\left[\left(W_{1}+W_{2}\right)^{2^{*}-1}-W_{1}^{2^{*}-1}-W_{2}^{2^{*}-1}-\left(2^{*}-1\right) W_{2}^{2^{*}-2} W_{1}\right] Z_{2, j} d v_{g}
\end{aligned}
$$

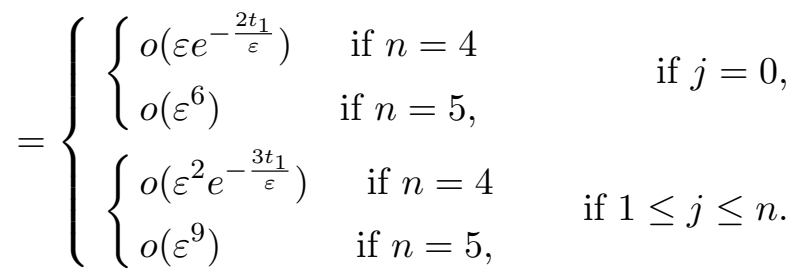

We now estimate the components in $(6.16)$ where $\phi_{\varepsilon}$ appears:

Claim 6.3. There holds, as $\varepsilon \rightarrow 0$, in $C^{0}\left(A_{1}\right)$ :

$$
\begin{aligned}
& \int_{M}\left[\left(W_{1}+W_{2}+\phi_{\varepsilon}\right)^{2^{*}-1}-\left(W_{1}+W_{2}\right)^{2^{*}-1}-\left(2^{*}-1\right)\left(W_{1}+W_{2}\right)^{2^{*}-2} \phi_{\varepsilon}\right] Z_{2, j} d v_{g} \\
& =\left\{\begin{array}{l} 
\begin{cases}o\left(\varepsilon e^{-\frac{2 t_{1}}{\varepsilon}}\right) & \text { if } n=4 \\
o\left(\varepsilon^{6}\right) & \text { if } n=5,\end{cases} \\
\left\{\begin{array}{ll}
o\left(\varepsilon^{2} e^{\left.-\frac{3 t_{1}}{\varepsilon}\right)}\right. & \text { if } n=4 \\
o\left(\varepsilon^{9}\right) & \text { if } n=5,
\end{array} \quad \text { if } 1 \leq j \leq n .\right.
\end{array}\right.
\end{aligned}
$$

Proof. Let $0<\varepsilon \leq \varepsilon_{2}$. By Proposition 4.1 we can write that there holds, in $M$ :

$$
\begin{array}{r}
\left|\left(W_{1}+W_{2}+\phi_{\varepsilon}\right)^{2^{*}-1}-\left(W_{1}+W_{2}\right)^{2^{*}-1}-\left(2^{*}-1\right)\left(W_{1}+W_{2}\right)^{2^{*}-2} \phi_{\varepsilon}\right| \\
\lesssim\left(W_{1}+W_{2}\right)^{2^{*}-3}\left|\phi_{\varepsilon}\right|^{2} .
\end{array}
$$

On one hand, using (3.1), (3.2), 4.4) and (6.26) we get that:

$$
\begin{aligned}
& \int_{B_{g}\left(\xi_{2}, 2 r_{0}\right) \backslash B_{g}\left(\xi_{2}, \frac{r_{0}}{2}\right)}\left[\left(W_{1}+W_{2}+\phi_{\varepsilon}\right)^{2^{*}-1}-\left(W_{1}+W_{2}\right)^{2^{*}-1}-\left(2^{*}-1\right)\left(W_{1}+W_{2}\right)^{2^{*}-2} \phi_{\varepsilon}\right] Z_{2, j} d v_{g} \\
& = \begin{cases} \begin{cases}o\left(\varepsilon e^{-\frac{2 t_{1}}{\varepsilon}}\right) & \text { if } n=4 \\
o\left(\varepsilon^{6}\right) & \text { if } n=5,\end{cases} & \text { if } j=0, \\
\begin{cases}o\left(\varepsilon^{2} e^{-\frac{3 t_{1}}{\varepsilon}}\right) & \text { if } n=4 \\
o\left(\varepsilon^{9}\right) & \text { if } n=5,\end{cases} & \text { if } 1 \leq j \leq n .\end{cases}
\end{aligned}
$$


On the other hand, using (5.2) and (5.3) to estimate $\left|\phi_{\varepsilon}\right|^{2}$ pointwise gives, with (3.1), (3.2) and 6.26), that:

$$
\begin{aligned}
& \int_{B_{g}\left(\xi_{2}, \frac{r_{0}}{2}\right)}\left[\left(W_{1}+W_{2}+\phi_{\varepsilon}\right)^{2^{*}-1}-\left(W_{1}+W_{2}\right)^{2^{*}-1}-\left(2^{*}-1\right)\left(W_{1}+W_{2}\right)^{2^{*}-2} \phi_{\varepsilon}\right] Z_{2, j} d v_{g} \\
& =\left\{\begin{array}{l}
\left\{\begin{array}{ll}
o\left(\varepsilon e^{-\frac{2 t_{1}}{\varepsilon}}\right) & \text { if } n=4 \\
o\left(\varepsilon^{6}\right) & \text { if } n=5,
\end{array} \quad \text { if } j=0,\right. \\
\left\{\begin{array}{ll}
o\left(\varepsilon^{2} e^{-\frac{3 t_{1}}{\varepsilon}}\right) & \text { if } n=4 \\
o\left(\varepsilon^{9}\right) & \text { if } n=5,
\end{array} \quad \text { if } 1 \leq j \leq n .\right.
\end{array}\right.
\end{aligned}
$$

Since by construction $Z_{2, j}$ is supported in $B_{g}\left(\xi_{2}, 2 r_{0}\right)$, combining the latter estimates gives 6.25.

It is important to remark that the high precision of $(5.2)$ and $(5.3)$ is really needed to estimate the integral in (6.25). The energy estimate (3.8) alone would fail: in dimension 4 , for instance, it would just yield:

$$
\int_{M} W_{2}\left|\phi_{\varepsilon}\right|^{2}\left|Z_{2, j}\right| d v_{g} \lesssim \varepsilon^{2} e^{-\frac{2 t_{1}}{\varepsilon}}
$$

which is not precise enough. Similarly, (4.4) alone would not be enough. Moving on, we have:

Claim 6.4. There holds, as $\varepsilon \rightarrow 0$, in $C^{0}\left(A_{1}\right)$ :

$$
\begin{array}{r}
\int_{M}\left[\left(\triangle_{g}+c_{n} S_{g}+h\right) \phi_{\varepsilon}-\left(2^{*}-1\right) W_{2}^{2^{*}-2} \phi_{\varepsilon}\right] Z_{2, j} d v_{g} \\
=\left\{\begin{array}{l}
\left\{\begin{array}{ll}
o\left(\varepsilon e^{-\frac{2 t_{1}}{\varepsilon}}\right) & \text { if } n=4 \\
o\left(\varepsilon^{6}\right) & \text { if } n=5,
\end{array} \quad \text { if } j=0,\right. \\
\left\{\begin{array}{ll}
o\left(\varepsilon^{2} e^{-\frac{3 t_{1}}{\varepsilon}}\right) & \text { if } n=4 \\
o\left(\varepsilon^{9}\right) & \text { if } n=5,
\end{array} \quad \text { if } 1 \leq j \leq n .\right.
\end{array}\right.
\end{array}
$$

Proof. Integrating by parts yields:

$$
\begin{aligned}
\int_{M} & {\left[\left(\triangle_{g}+c_{n} S_{g}+h\right) \phi_{\varepsilon}-\left(2^{*}-1\right) W_{2}^{2^{*}-2} \phi_{\varepsilon}\right] Z_{2, j} d v_{g} } \\
& =\int_{B_{g}\left(\xi_{2}, 2 r_{0}\right)}\left[\left(\triangle_{g}+c_{n} S_{g}+h\right) Z_{2, j}-\left(2^{*}-1\right) W_{2}^{2^{*}-2} Z_{2, j}\right] \phi_{\varepsilon} d v_{g}
\end{aligned}
$$


As in the proof of (6.25), the latter integral is split into two pieces. First, the contribution on $B_{g}\left(\xi_{2}, 2 r_{0}\right) \backslash B_{g}\left(\xi_{2}, \frac{r_{0}}{2}\right)$ is computed using (3.1), (3.2) and 4.4) and yields:

$$
\begin{aligned}
& \int_{B_{g}\left(\xi_{2}, 2 r_{0}\right) \backslash B_{g}\left(\xi_{2}, \frac{r_{0}}{2}\right)}\left[\left(\triangle_{g}+c_{n} S_{g}+h\right) Z_{2, j}-\left(2^{*}-1\right) W_{2}^{2^{*}-2} Z_{2, j}\right] \phi_{\varepsilon} d v_{g} \\
& =\left\{\begin{array}{l} 
\begin{cases}o\left(\varepsilon e^{-\frac{2 t_{1}}{\varepsilon}}\right) & \text { if } n=4 \\
o\left(\varepsilon^{6}\right) & \text { if } n=5,\end{cases} \\
\left\{\begin{array}{ll}
o\left(\varepsilon^{2} e^{\left.-\frac{3 t_{1}}{\varepsilon}\right)}\right. & \text { if } n=4 \\
o\left(\varepsilon^{9}\right) & \text { if } n=5,
\end{array} \quad \text { if } 1 \leq j \leq n .\right.
\end{array}\right.
\end{aligned}
$$

The contribution on $B_{g}\left(\xi_{2}, \frac{r_{0}}{2}\right)$ is estimated using (3.1), (3.2), 5.2), (5.3), 5.24) and 5.37) and yields again:

$$
\begin{aligned}
& \int_{B_{g}\left(\xi_{2}, \frac{r_{0}}{2}\right)}\left[\left(\triangle_{g}+c_{n} S_{g}+h\right) Z_{2, j}-\left(2^{*}-1\right) W_{2}^{2^{*}-2} Z_{2, j}\right] \phi_{\varepsilon} d v_{g} \\
& =\left\{\begin{array}{l}
\left\{\begin{array}{ll}
o\left(\varepsilon e^{-\frac{2 t_{1}}{\varepsilon}}\right) & \text { if } n=4 \\
o\left(\varepsilon^{6}\right) & \text { if } n=5,
\end{array} \quad \text { if } j=0,\right. \\
\left\{\begin{array}{ll}
o\left(\varepsilon^{2} e^{-\frac{3 t_{1}}{\varepsilon}}\right) & \text { if } n=4 \\
o\left(\varepsilon^{9}\right) & \text { if } n=5,
\end{array} \quad \text { if } 1 \leq j \leq n,\right.
\end{array}\right.
\end{aligned}
$$

which concludes the proof of 6.27.

The last term appearing in the expansion of the $\lambda_{i, j}^{\varepsilon}$ is estimated in the same way:

Claim 6.5. There holds, as $\varepsilon \rightarrow 0$, in $C^{0}\left(A_{1}\right)$ :

$$
\begin{gathered}
-\int_{M}\left(2^{*}-1\right)\left[\left(W_{1}+W_{2}\right)^{2^{*}-2}-W_{2}^{2^{*}-2}\right] \phi_{\varepsilon} Z_{2, j} d v_{g} \\
=\left\{\begin{array}{l}
\left\{\begin{array}{ll}
o\left(\varepsilon e^{-\frac{2 t_{1}}{\varepsilon}}\right) & \text { if } n=4 \\
o\left(\varepsilon^{6}\right) & \text { if } n=5,
\end{array} \quad \text { if } j=0,\right. \\
\left\{\begin{array}{ll}
o\left(\varepsilon^{2} e^{-\frac{3 t_{1}}{\varepsilon}}\right) & \text { if } n=4 \\
o\left(\varepsilon^{9}\right) & \text { if } n=5,
\end{array} \quad \text { if } 1 \leq j \leq n .\right.
\end{array}\right.
\end{gathered}
$$

Proof. We write that:

$$
\left|\left(W_{1}+W_{2}\right)^{2^{*}-2}-W_{2}^{2^{*}-2}\right| \lesssim W_{2}^{2^{*}-3} W_{1}+W_{1}^{2^{*}-2} .
$$

The claim follows then from the same computations that led to 6.25$)$ and $(6.27)$. The contribution of the integral on $B_{g}\left(\xi_{2}, 2 r_{0}\right) \backslash B_{g}\left(\xi_{2}, \frac{r_{0}}{2}\right)$ is estimated with (3.1), (3.2), (4.4) and $(6.29)$, while the contribution on $B_{g}\left(\xi_{2}, \frac{r_{0}}{2}\right)$ is estimated with (3.1), (3.2), (5.2), (5.3) and $(6.29)$. 
To conclude the proof of the Lemma it remains to notice that by $(3.2)$ there holds, as before:

$$
\left\langle Z_{2, j}, Z_{2, k}\right\rangle=\delta_{j k}\left\|\nabla V_{j}\right\|_{L^{2}\left(\mathbb{R}^{n}\right)}^{2}+o(1)
$$

for $0 \leq j, k \leq n$, and even:

$$
\left\langle Z_{2,0}, Z_{2, j}\right\rangle= \begin{cases}o\left(\varepsilon e^{-\frac{t_{1}}{\varepsilon}}\right) & \text { if } n=4 \\ o\left(\varepsilon^{3}\right) & \text { if } n=5,\end{cases}
$$

for $1 \leq j \leq n$, in $C^{0}\left(A_{1}\right)$ as $\varepsilon \rightarrow 0$, where $\langle\cdot, \cdot\rangle$ is as in (3.6) and $V_{j}$ is as in (5.22). Combining the latter with (6.17), (6.18), (6.19), (6.21), (6.22), (6.24), (6.25), (6.27) and 6.28 concludes the proof of the Lemma.

The expansions obtained in Lemmas 6.1 and 6.2 hold in $C^{0}\left(A_{1}\right)$, and this is a consequence of the uniformity of the estimates $(4.4), 5.2)$ and $(5.3)$.

\section{Concluding ARgument}

We assume now that the assumptions of Theorems 1.1 and 1.2 are satisfied: we let $\xi_{1,0} \neq \xi_{2,0}$ be distinct points in $M$ and assume that $h=h_{\delta}$ is given by (1.4), where $\Psi$ is as described in the discussion before (1.4). If $(M, g)$ is conformally diffeomorphic to the standard sphere we assume in addition that $\int_{\mathbb{R}^{n}} \Psi(y) d y<0$. Our choice of $H$ will be detailed below. We conclude in this section the proof of Theorems 1.1 and 1.2 by showing the existence, for any $\varepsilon>0$ small enough, of an element $\left(t_{1, \varepsilon}, \xi_{1, \varepsilon}, t_{2, \varepsilon}, \xi_{2, \varepsilon}\right)$ that annihilates the $\lambda_{i, j}^{\varepsilon}$ in 4.19. The family $\left(u_{\varepsilon}\right)_{\varepsilon}$ we are looking for will then be given by

$$
u_{\varepsilon}=W_{1}+W_{2}+\phi_{\varepsilon}\left(t_{1, \varepsilon}, \xi_{1, \varepsilon}, t_{2, \varepsilon}, \xi_{2, \varepsilon}\right),
$$

using the notations of (4.2). Its positivity and its smoothness follow from standard arguments.

For any $0<\delta \leq 1$, we let $U_{\delta}(y)=G_{h_{\delta}}\left(\xi_{1,0}, y\right)-G_{g}\left(\xi_{1,0}, y\right)$, where $G_{h_{\delta}}$ and $G_{g}$ are respectively the Green functions of $\triangle_{g}+c_{n} S_{g}+h_{\delta}$ and $\triangle_{g}+c_{n} S_{g}$. It satisfies

$$
\left(\triangle_{g}+c_{n} S_{g}+h_{\delta}\right) U_{\delta}=-h_{\delta} G_{g}\left(\xi_{1,0}, \cdot\right) \text {. }
$$

Since for $\delta$ small enough the support of $h_{\delta}$ does not meet $\xi_{1,0}$, standard elliptic regularity theory shows that $U_{\delta} \in C^{2}(M)$ and that:

$$
\left\|U_{\delta}\right\|_{C^{1}(M)} \lesssim \delta, \quad\left\|U_{\delta}\right\|_{C^{2}(M)} \lesssim \frac{1}{\delta}
$$

for all $0<\delta \leq 1$. Standard elliptic theory again shows that, for any $0<\delta<1$, $\frac{d U_{\delta}}{d \delta}$ exists, is in $C^{1}(M)$ and satisfies:

$$
\delta\left\|\frac{d U_{\delta}}{d \delta}\right\|_{C^{1}(M)} \rightarrow 0
$$

as $\delta \rightarrow 0$. We will only prove Theorems 1.1 and 1.2 in case $n=4$. The proof for the $n=5$ case follows from minor modifications and we omit it here. We will show that the 
following mapping:

$$
F_{4}^{\delta}\left(t_{1}, \xi_{1}, t_{2}, \xi_{2}\right)=\left(\begin{array}{l}
C_{1}(4) H\left(\xi_{1}\right) t_{1}-C_{2}(4) A_{h_{\delta}}\left(\xi_{1}\right)-\frac{C_{3}(4)}{2} F_{h_{\delta}}\left(\xi_{1}\right) \\
C_{1}(4) \nabla H\left(\xi_{1}\right) t_{1}-C_{2}(4) \nabla A_{h_{\delta}}\left(\xi_{1}\right) \\
2 D_{1}(4) h\left(\xi_{2}\right) t_{1} t_{2}^{2}-D_{2}(4) t_{2} G_{h_{\delta}}\left(\xi_{1}, \xi_{2}\right) \\
D_{1}(4) \nabla h\left(\xi_{2}\right) t_{1} t_{2}^{3}-D_{2}(4) t_{2}^{2} \nabla G_{h_{\delta}}\left(\xi_{1}, \xi_{2}\right)
\end{array}\right)
$$

possesses a non-degenerate zero for any $\delta$ small enough, where

$$
A_{h_{\delta}}\left(\xi_{1}\right)=A\left(\xi_{1}\right)-\frac{C_{3}(4)}{C_{2}(4)} F_{h_{\delta}}\left(\xi_{1}\right)
$$

is the non-local mass induced by $h_{\delta}, A$ is the Riemannian mass introduced in (2.4) and $F_{h_{\delta}}$ is defined in 6.3). In view of Lemmas 6.1 and 6.2, and with a standard degree argument, this will yield the existence, for any small $\varepsilon$, of $\left(t_{1, \varepsilon}, \xi_{1, \varepsilon}, t_{2, \varepsilon}, \xi_{2, \varepsilon}\right)$ annihilating all the $\lambda_{i, j}^{\varepsilon}$ in 4.19 .

We start by defining the following mapping:

$$
\varphi:\left\{\begin{array}{l}
{[0,1) \times B_{0}(1) \rightarrow \mathbb{R}^{n}} \\
(\delta, y) \mapsto \frac{\nabla \Psi(y)}{\Psi(y)}-2 \frac{\nabla\left(G_{h_{\delta}}\left(\xi_{1,0}, \exp _{\xi_{2,0}}(\delta \cdot)\right)\right)(y)}{G_{h_{\delta}}\left(\xi_{1,0}, \exp _{\xi_{2,0}}(\delta y)\right)}
\end{array}\right.
$$

It is easily seen with 7.1 and 7.2 that $\varphi$ is of class $C^{1}$. By the choice of $\Psi$ there holds $\varphi(0,0)=0$ and

$$
D \varphi(0,0)=\frac{1}{\Psi(0)} \nabla^{2} \Psi(0),
$$

which is non-degenerate. The implicit function theorem applies then and yields a continuous path $y_{\delta} \in B_{0}(1)$, defined for any $0<\delta \leq \delta_{0}$, such that $\varphi\left(\delta, y_{\delta}\right)=0$ and $y_{\delta} \rightarrow 0$ as $\delta \rightarrow 0$. Letting $\xi_{2, \delta}=\exp _{\xi_{2,0}}\left(\delta y_{\delta}\right)$ and noting that, by (1.4), there holds $h_{\delta}\left(\exp _{\xi_{2,0}}(\delta y)\right)=\Psi(y)$, it is easily seen that for any $\delta>0$ small enough $\xi_{2, \delta}$ satisfies

$$
\frac{\nabla h_{\delta}\left(\xi_{2, \delta}\right)}{h_{\delta}\left(\xi_{2, \delta}\right)}=2 \frac{\nabla G_{h_{\delta}}\left(\xi_{1,0}, \cdot\right)\left(\xi_{2, \delta}\right)}{G_{h_{\delta}}\left(\xi_{1,0}, \xi_{2, \delta}\right)}
$$

In addition we have

$$
d_{g}\left(\xi_{2,0}, \xi_{2, \delta}\right)=o(\delta) \text { as } \delta \rightarrow 0,
$$

so that by 1.4 there holds:

$$
\begin{aligned}
& h_{\delta}\left(\xi_{2, \delta}\right)=\Psi(0)+o(1), \quad\left|\nabla h_{\delta}\left(\xi_{2, \delta}\right)\right|_{g}=o(1) \text { and } \\
& \nabla^{2} h_{\delta}\left(\xi_{2, \delta}\right)=\frac{1}{\delta^{2}} \nabla^{2} \Psi(0)+O\left(\frac{1}{\delta}\right) \text { as } \delta \rightarrow 0 .
\end{aligned}
$$

We now choose $H$ such that

$$
H\left(\xi_{1,0}\right)=A_{h_{\delta}}\left(\xi_{1,0}\right)+\frac{C_{3}(4)}{2 C_{2}(4)} F_{h_{\delta}}\left(\xi_{1,0}\right), \quad \nabla H\left(\xi_{1,0}\right)=\nabla A_{h_{\delta}}\left(\xi_{1,0}\right),
$$

where $A_{h_{\delta}}$ is defined in (7.4), and such that

the matrix $\nabla_{\left(t_{1}, \xi_{1}\right)}\left(F_{4}^{\delta}\right)_{1 \leq j \leq 5}\left(t_{1,0}, \xi_{1,0}\right)$ is non-degenerate for all $0<\delta \leq \delta_{0}$, 
where $F_{4}^{\delta}$ is defined in $(7.3)$. In the latter condition one could assume that $\delta=0$ - at least when $A>0$ - since $A_{h_{\delta}}$ converges to $A$ in $C^{2}(M)$ as $\delta \rightarrow 0$. With this choice of $H$ there always holds that $H\left(\xi_{1,0}\right)>0$. This is clear for small $\delta$ when $(M, g)$ is not conformally diffeomorphic to $\left(\mathbb{S}^{n}, g_{\text {std }}\right)$ since in this case $A>0$. In the case where $(M, g)$ is the standard sphere $\left(\mathbb{S}^{n}, g_{s t d}\right)$ there holds, with (6.3) and (7.1), that:

$$
\begin{aligned}
A_{h_{\delta}}\left(\xi_{1,0}\right)+\frac{C_{3}(4)}{2 C_{2}(4)} F_{h_{\delta}}\left(\xi_{1,0}\right) & =-\frac{C_{3}(4)}{2 C_{2}(4)} F_{h_{\delta}}\left(\xi_{1,0}\right) \\
& =-\frac{C_{3}(4)}{2 C_{2}(4)} G_{g}\left(\xi_{1,0}, \xi_{2,0}\right)^{2} \int_{\mathbb{R}^{4}} \Psi(y) d y \cdot \delta^{4}+O\left(\delta^{5}\right),
\end{aligned}
$$

which is positive for small $\delta$ by the assumption on $\Psi$. It remains to define:

$$
t_{1,0}=\frac{C_{2}(4)}{C_{1}(4)} \text { and } t_{2, \delta}=\frac{D_{2}(4)}{2 D_{1}(4)} \frac{G_{h_{\delta}}\left(\xi_{1,0}, \xi_{2, \delta}\right)}{t_{1,0} h_{\delta}\left(\xi_{2, \delta}\right)} .
$$

With (7.1) and (7.6), it is easily seen that $t_{2, \delta}$ converges towards a positive limit as $\delta \rightarrow 0$. Using (7.5), (7.7) and (7.9) it is easily seen that $\left(t_{1,0}, \xi_{1,0}, t_{2, \delta}, \xi_{2, \delta}\right)$ is a zero of $F_{4}^{\delta}$ defined in 7.3 for $0<\delta \leq \delta_{0}$.

It remains to show that this zero is non-degenerate. With $(7.9)$ there holds:

$$
\partial_{t_{2}}\left(F_{4}^{\delta}\right)_{6}\left(t_{1,0}, \xi_{1,0}, t_{2, \delta}, \xi_{2, \delta}\right)=D_{2}(4) G_{h_{\delta}}\left(\xi_{1,0}, \xi_{2, \delta}\right)>0
$$

while 7.5 and $(7.6)$ show that

$$
\partial_{t_{2}}\left(F_{4}^{\delta}\right)_{7 \leq j \leq 10}\left(t_{1,0}, \xi_{1,0}, t_{2, \delta}, \xi_{2, \delta}\right)=o(1) \text { as } \delta \rightarrow 0,
$$

and that

$$
\nabla_{\xi_{2}}\left(F_{4}^{\delta}\right)_{6}\left(t_{1,0}, \xi_{1,0}, t_{2, \delta}, \xi_{2, \delta}\right)=o(1) \text { as } \delta \rightarrow 0 .
$$

Finally, (7.1) and (7.6) show that

$$
\nabla_{\xi_{2}}\left(F_{4}^{\delta}\right)_{7 \leq j \leq 10}\left(t_{1,0}, \xi_{1,0}, t_{2, \delta}, \xi_{2, \delta}\right)=D_{1}(4) t_{1,0} t_{2,0}^{3} \frac{1}{\delta^{2}} \nabla^{2} \Psi(0)+O\left(\frac{1}{\delta}\right) \text { as } \delta \rightarrow 0 .
$$

Together with 7.8$)$ this shows that $\left(t_{1,0}, \xi_{1,0}, t_{2, \delta}, \xi_{2, \delta}\right)$ is a non-degenerate zero of $F_{4}^{\delta}$ for all $\delta$ small enough and concludes the proof of Theorems 1.1 and 1.2 .

Remark 7.1. The choice of $h$ as in (1.4) only comes into play in Section 7. The statement of Theorems 1.1 and 1.2 obviously remains true for other choices of $h$ than (1.4), provided one can find a zero of $F_{4}^{\delta}$ in $(7.3)$ of nontrivial degree. We mention another situation where this is true: assume that $h$ is not too large and has a critical point with small hessian at $\xi_{2,0}$. Then, simple considerations show that a non-degenerate zero of $F_{4}^{\delta}$ exists provided $\xi_{2,0}$ is a non-degenerate critical point of the function

$$
\xi_{2} \mapsto G_{g}\left(\xi_{1,0}, \xi_{2}\right)
$$

A minimum for the latter function always exists, and its non-degeneracy is generic in the choice of $g$ in a given conformal class. This yields another situation where the family $\left(u_{\varepsilon}\right)_{\varepsilon}$ in the statement of Theorems 1.1 and 1.2 generically exists. 


\section{APPENDIX A. A PRIORI BLOW-UP ANALYSIS}

In this Appendix we show how the bubbling configuration of Theorems 1.1 and 1.2 - and in particular relation $(1.9)$ - is predicted by an a priori blow-up analysis for equation (1.5). For this, we assume that $h$ and $H$ are functions as in Section 2 and we let $\left(u_{k}\right)_{k}$ be a sequence of positive solutions of:

$$
\triangle_{g} u_{k}+\left(c_{n} S_{g}+h+\varepsilon_{k} H\right) u_{k}=u_{k}^{2^{*}-1}
$$

in $M$, where $\left(\varepsilon_{k}\right)_{k}$ is a sequence of positive numbers such that $\lim _{k \rightarrow+\infty} \varepsilon_{k}=0$. We assume that $\left(u_{k}\right)_{k}$ blows-up with two bubbles, that is $\lim _{k \rightarrow+\infty}\left\|u_{k}\right\|_{C^{0}(M)}=+\infty$ and $\lim _{k \rightarrow+\infty}\left\|u_{k}\right\|_{L^{2^{*}(M)}}=2 K_{n}^{-\frac{n-2}{2}}$, where $K_{n}$ is defined in 4.6). The $H^{1}$-theory of [26] asserts the existence of sequences $\left(\mu_{1, k}\right)_{k}$ and $\left(\mu_{2, k}\right)_{k}$ of positive numbers converging to 0 and of sequences $\left(\xi_{1, k}\right)_{k}$ and $\left(\xi_{2, k}\right)_{k}$ of points of $M$ converging towards $\xi_{1}$ and $\xi_{2}$ such that:

$$
u_{k}=B_{1, k}+B_{2, k}+o(1) \text { in } H^{1}(M),
$$

where we have let, for $i=1,2$ and for any $x \in M$ :

$$
B_{i}(x)=\left(\frac{\mu_{i, k}}{\mu_{i, k}^{2}+\frac{d_{g}\left(\xi_{i, k}, x\right)^{2}}{n(n-2)}}\right)^{\frac{n-2}{2}} .
$$

Note that we do not assume a priori that the concentration points are isolated, that is that $\xi_{1} \neq \xi_{2}$. This will be obtained as a consequence of the assumption $h\left(\xi_{2}\right)>0$. By convention, we assume that $B_{1, k}$ denotes the lowest bubble, that is $\mu_{1, k} \geq \mu_{2, k}$. Let $G_{h}$ denote the Green's function of $\triangle_{g}+c_{n} S_{g}+h$ in $M$. Define, for any $x, y \in M$ :

$$
\Phi_{k}(x, y)=(n-2) \omega_{n-1} d_{g}(x, y)^{n-2} G_{h}(x, y) .
$$

It is a positive continuous function in $M$. The $C^{0}$-theory of [7] (see also [9], Proposition 7.2) improves Struwe's $H^{1}$ decomposition into a global $C^{0}$ one and shows that there exists a sequence $\left(\eta_{k}\right)_{k}$ of positive numbers with $\lim _{k \rightarrow+\infty} \eta_{k}=0$ such that:

$$
\left\|\frac{u_{k}-\Phi_{h}\left(\xi_{1}, \cdot\right) B_{1, k}-\Phi_{h}\left(\xi_{2}, \cdot\right) B_{2, k}}{B_{1, k}+B_{2, k}}\right\|_{C^{0}(M)} \leq \eta_{k} .
$$

First, as a consequence of $(\mathrm{A} .2)$ and of the analysis of [5] (see also the proof of Theorem 8.1 in [9]), it turns out that $h\left(\xi_{1}\right)=0$. That is: the lowest bubble concentrates at a point where the limiting potential $c_{n} S_{g}+h$ touches the geometric thresholds $c_{n} S_{g}$. If we now assume that $h\left(\xi_{2}\right)>0$ we get that $d_{g}\left(\xi_{1}, \xi_{2}\right)>0$. We now investigate necessary conditions on the highest bubble $B_{2, k}$. Let

$$
r_{2, k}=\min \left(\sqrt{\frac{\mu_{2, k}}{\mu_{1, k}} \frac{d_{g}\left(\xi_{1, k}, \xi_{2, k}\right)^{2}}{n(n-2)}}, \frac{1}{2} i_{g}(M)\right)
$$

be the radius of influence of the bubble $B_{2, k}$, as introduced in [5]. We recall that by the analysis in [26] there holds:

$$
\frac{\mu_{1, k}}{\mu_{2, k}}+\frac{\mu_{2, k}}{\mu_{1, k}}+\frac{d_{g}\left(\xi_{1, k}, \xi_{2, k}\right)^{2}}{\mu_{1, k} \mu_{2, k}} \rightarrow+\infty
$$


as $k \rightarrow+\infty$. In what follows we let $\Omega_{k}=B_{g}\left(\xi_{2, k}, r_{2, k}\right)$ and write down a Pohozaev identity in $\Omega_{k}$ for $u_{k}$. We will only sketch the arguments since most of the material is taken from [9]. Let $X_{k}$ be the following field of 1-forms in $\Omega_{k}$ :

$$
X_{k}(x)=\left(1-\frac{1}{6(n-1)} \operatorname{Ric}^{\sharp}(x)\left(\nabla d_{k}(x), \nabla d_{k}(x)\right)\right) \nabla d_{k}(x),
$$

where $d_{k}(x)=d_{g}\left(\xi_{2, k}, x\right)$. Multiplying (A.1) by $X_{k}\left(u_{k}\right)$ leads to the following Pohozaev identity in $\Omega_{k}$ (see [9], Proposition 6.2):

$$
\begin{aligned}
& \int_{\Omega_{k}}\left(c_{n} S_{g}+h\right) u_{k} X_{k}^{p} \nabla_{p} u_{k} d v_{g}+\frac{n-2}{4 n} \int_{\Omega_{k}} \triangle_{g}\left(\operatorname{div}_{g} X_{k}\right) u_{k}^{2} d v_{g} \\
& \quad+\frac{n-2}{2 n} \int_{\Omega_{k}} \operatorname{div}_{g} X_{k}\left(c_{n} S_{g}+h\right) u_{k}^{2} d v_{g} \\
& =-\int_{\Omega_{k}}\left(\nabla X_{k}-\frac{1}{n} \operatorname{div}_{g} X_{k} g\right)\left(\nabla u_{k}, \nabla u_{k}\right) d v_{g} \\
& +\frac{n-2}{2 n} \int_{\partial \Omega_{k}} X_{k}(\nu) u_{k}^{2^{*}} d \sigma_{g}-\frac{n-2}{4 n} \int_{\partial \Omega_{k}} \partial_{\nu}\left(\operatorname{div}_{g} X_{k}\right) u_{k}^{2} d \sigma_{g} \\
& \quad-\int_{\partial \Omega_{k}}\left(-\frac{n-2}{2 n} \operatorname{div}_{g} X_{k} \partial_{\nu} u_{k} u_{k}+\frac{1}{2} X_{k}(\nu)\left|\nabla u_{k}\right|^{2}-X_{k}^{p} \nabla_{p} u_{k} \partial_{\nu} u_{k}\right) d \sigma_{g} .
\end{aligned}
$$

In (A.4) we used that $H$ vanishes on $\Omega_{k}$. Now, and adaptation of the arguments in 9 (Lemma 6.4) using A.2 shows that:

$$
\int_{\Omega_{k}}\left(\nabla X_{k}-\frac{1}{n} \operatorname{div}_{g} X_{k} g\right)\left(\nabla u_{k}, \nabla u_{k}\right) d v_{g}=O\left(\mu_{2, k}^{n-2} r_{2, k}^{4-n}\right)+ \begin{cases}O\left(\mu_{2, k}^{2}\right) & \text { if } n=4 \\ o\left(\mu_{2, k}^{2}\right) & \text { if } n=5\end{cases}
$$

and that

$$
\begin{aligned}
& \int_{\Omega_{k}}\left(c_{n} S_{g}+h\right) u_{k} X_{k}^{p} \nabla_{p} u_{k} d v_{g}+\frac{n-2}{4 n} \int_{\Omega_{k}} \triangle_{g}\left(\operatorname{div}_{g} X_{k}\right) u_{k}^{2} d v_{g}+\frac{n-2}{2 n} \int_{\Omega_{k}} \operatorname{div}_{g} X_{k}\left(c_{n} S_{g}+h\right) u_{k}^{2} d v_{g} \\
& =-D_{1}(n) h\left(\xi_{2, k}\right) \cdot \begin{cases}\mu_{2, k}^{2}\left|\ln \mu_{2, k}\right|+O\left(\mu_{2, k}^{2}\right) & \text { if } n=4 \\
\mu_{2, k}^{2}+o\left(\mu_{2, k}^{2}\right) & \text { if } n=5\end{cases}
\end{aligned}
$$

for some positive constant $D_{1}(n)$. Finally, there holds by $($ A.2 that

$$
\frac{n-2}{2 n} \int_{\partial \Omega_{k}} X_{k}(\nu) u_{k}^{2^{*}} d \sigma_{g}-\frac{n-2}{4 n} \int_{\partial \Omega_{k}} \partial_{\nu}\left(\operatorname{div}_{g} X_{k}\right) u_{k}^{2} d \sigma_{g}=O\left(\mu_{2, k}^{n} r_{2, k}^{-n}\right)+O\left(\mu_{2, k}^{n-2} r_{2, k}^{4-n}\right)
$$

and, using the definition of $X_{k}$, that

$$
\begin{aligned}
& -\int_{\partial \Omega_{k}}\left(-\frac{n-2}{2 n} \operatorname{div}_{g} X_{k} \partial_{\nu} u_{k} u_{k}+\frac{1}{2} X_{k}(\nu)\left|\nabla u_{k}\right|^{2}-X_{k}^{p} \nabla_{p} u_{k} \partial_{\nu} u_{k}\right) d \sigma_{g} \\
& =-\int_{\partial \Omega_{k}}\left(-\frac{n-2}{2} \partial_{\nu} u_{k} u_{k}+\frac{1}{2} X_{k}(\nu)\left|\nabla u_{k}\right|^{2}-X_{k}^{p} \nabla_{p} u_{k} \partial_{\nu} u_{k}\right) d \sigma_{g}+O\left(\mu_{2, k}^{n-2} r_{2, k}^{4-n}\right)
\end{aligned}
$$


With A.2, it is easily seen that there holds:

$$
\mu_{2, k}^{1-\frac{n}{2}} r_{2, k}^{n-2} u_{k}\left(\exp _{\xi_{2, k}}\left(r_{2, k} \cdot\right)\right) \rightarrow \frac{(n(n-2))^{\frac{n-2}{2}}}{|x|^{n-2}}+\Phi_{h}\left(\xi_{1}, \xi_{2}\right)
$$

in $C_{l o c}^{2}\left(B_{0}(1) \backslash\{0\}\right)$. The latter convergence, with (A.8), now shows that:

$$
\begin{aligned}
& -\int_{\partial \Omega_{k}}\left(-\frac{n-2}{2 n} \operatorname{div}_{g} X_{k} \partial_{\nu} u_{k} u_{k}+\frac{1}{2} X_{k}(\nu)\left|\nabla u_{k}\right|^{2}-X_{k}^{p} \nabla_{p} u_{k} \partial_{\nu} u_{k}\right) d \sigma_{g} \\
& =\left(-\frac{(n-2)^{2}}{2}(n(n-2))^{\frac{n-2}{2}} \omega_{n-1} \Phi_{h}\left(\xi_{1}, \xi_{2}\right)+o(1)\right)\left(\frac{\mu_{2, k}}{r_{2, k}}\right)^{n-2} .
\end{aligned}
$$

Combining (A.5), A.6, A.7) and A.9 in A.4 and using (A.3) then gives:

$$
\begin{array}{ll}
\left(h\left(\xi_{2}\right)+o(1)\right) \mu_{2, k}^{2}\left|\ln \mu_{2, k}\right|=\left(\alpha(4) G_{h}\left(\xi_{1}, \xi_{2}\right)+o(1)\right) \mu_{1, k} \mu_{2, k} & \text { if } n=4, \\
\left(h\left(\xi_{2}\right)+o(1)\right) \mu_{2, k}^{2}=\left(\alpha(5) G_{h}\left(\xi_{1}, \xi_{2}\right)+o(1)\right)\left(\mu_{1, k} \mu_{2, k}\right)^{\frac{3}{2}} & \text { if } n=5
\end{array}
$$

for some positive constant $\alpha(n)$, which is $(1.9)$. One can also prove that $(1.10)$ is a necessary condition to be satisfied by the lowest bubble of the decomposition. This follows again from a Pohozaev identity using the analysis of [5].

\section{REFERENCES}

1. Gabriele Bianchi and Henrik Egnell, A note on the Sobolev inequality, J. Funct. Anal. 100 (1991), no. 1, 18-24. MR 1124290 (92i:46033)

2. Simon Brendle and Fernando C. Marques, Blow-up phenomena for the Yamabe equation. II, J. Differential Geom. 81 (2009), no. 2, 225-250. MR 2472174 (2010k:53050)

3. Luis A. Caffarelli, Basilis Gidas, and Joel Spruck, Asymptotic symmetry and local behavior of semilinear elliptic equations with critical Sobolev growth, Comm. Pure Appl. Math. 42 (1989), no. 3, 271-297. MR 982351 (90c:35075)

4. Wenyi Chen, Juncheng Wei, and Shusen Yan, Infinitely many solutions for the Schrödinger equations in $\mathbb{R}^{N}$ with critical growth, J. Differential Equations 252 (2012), no. 3, 2425-2447. MR 2860624

5. Olivier Druet, From one bubble to several bubbles: the low-dimensional case, J. Differential Geom. 63 (2003), no. 3, 399-473. MR 2015469

6. __ Compactness for Yamabe metrics in low dimensions, Int. Math. Res. Not. (2004), no. 23, 1143-1191. MR 2041549 (2005b:53056)

7. Olivier Druet, Emmanuel Hebey, and Frédéric Robert, Blow-up theory for elliptic PDEs in Riemannian geometry, Mathematical Notes, vol. 45, Princeton University Press, Princeton, NJ, 2004. MR 2063399 (2005g:53058)

8. Pierpaolo Esposito, Angela Pistoia, and Jérôme Vétois, The effect of linear perturbations on the Yamabe problem, Math. Ann. 358 (2014), no. 1-2, 511-560. MR 3158007

9. Emmanuel Hebey, Compactness and stability for nonlinear elliptic equations, Zurich Lectures in Advanced Mathematics, European Mathematical Society (EMS), Zürich, 2014. MR 3235821

10. Emmanuel Hebey and Juncheng Wei, Resonant states for the static Klein-Gordon-Maxwell-Proca system, Math. Res. Lett. 19 (2012), no. 4, 953-967. MR 3008428

11. M. A. Khuri, F. C. Marques, and R. M. Schoen, A compactness theorem for the Yamabe problem, J. Differential Geom. 81 (2009), no. 1, 143-196. MR 2477893 (2010e:53065)

12. John M. Lee and Thomas H. Parker, The Yamabe problem, Bull. Amer. Math. Soc. (N.S.) 17 (1987), no. 1, 37-91. MR 888880 (88f:53001)

13. Yan Yan Li and Lei Zhang, Compactness of solutions to the Yamabe problem. III, J. Funct. Anal. 245 (2007), no. 2, 438-474. MR 2309836 (2008f:53038) 
14. Yanyan Li and Meijun Zhu, Yamabe type equations on three-dimensional Riemannian manifolds, Commun. Contemp. Math. 1 (1999), no. 1, 1-50. MR 1681811 (2000m:53051)

15. Fernando Coda Marques, A priori estimates for the Yamabe problem in the non-locally conformally flat case, J. Differential Geom. 71 (2005), no. 2, 315-346. MR 2197144 (2006i:53046)

16. Anna Maria Micheletti, Angela Pistoia, and Jérôme Vétois, Blow-up solutions for asymptotically critical elliptic equations on Riemannian manifolds, Indiana Univ. Math. J. 58 (2009), no. 4, 1719-1746. MR 2542977

17. Filippo Morabito, Angela Pistoia, and Giusi Vaira, Towering phenomena for the yamabe equation on symmetric manifolds, (2016), Preprint.

18. Tiancheng Ouyang, On the positive solutions of semilinear equations $\Delta u+\lambda u-h u^{p}=0$ on the compact manifolds, Trans. Amer. Math. Soc. 331 (1992), no. 2, 503-527. MR 1055810 (92h:35012)

19. Angela Pistoia and Giusi Vaira, Clustering phenomena for a linear perturbation of the yamabe equation, (2015), Preprint.

20. Angela Pistoia and Jérôme Vétois, Sign-changing bubble towers for asymptotically critical elliptic equations on Riemannian manifolds, J. Differential Equations 254 (2013), no. 11, 4245-4278. MR 3035432

21. Bruno Premoselli, A pointwise finite-dimensional reduction method for Einstein-Lichnerowicz-type systems: the six-dimensional case, Preprint, 16 pages.

22. __ A pointwise finite-dimensional reduction method for a fully coupled system of EinsteinLichnerowicz type, (2016), Preprint, 59 pages.

23. Olivier Rey and Juncheng Wei, Arbitrary number of positive solutions for an elliptic problem with critical nonlinearity, J. Eur. Math. Soc. (JEMS) 7 (2005), no. 4, 449-476. MR 2159223

24. Frédéric Robert, Existence et asymptotiques optimales des fonctions de Green des opérateurs elliptiques d'ordre deux, http://www.iecn.u-nancy.fr/ frobert/ConstrucGreen.pdf.

25. Frédéric Robert and Jérôme Vétois, A general theorem for the construction of blowing-up solutions to some elliptic nonlinear equations with Lyapunov-Schmidt's finite-dimensional reduction, Concentration Compactness and Profile Decomposition (Bangalore, 2011), Trends in Mathematics, Springer, Basel (2014), 85-116.

26. Michael Struwe, A global compactness result for elliptic boundary value problems involving limiting nonlinearities, Math. Z. 187 (1984), no. 4, 511-517. MR 760051

27. Pierre-Damien Thizy, The Lin-Ni conjecture in negative geometries, J. Differential Equations 260 (2016), no. 4, 3658-3690. MR 3434412

28. Phase stability for Schrödinger-Poisson critical systems in closed 5-manifolds, Int. Math. Res. Not. IMRN (2016), no. 20, 6245-6292. MR 3579965

29. Pierre-Damien Thizy and Jérôme Vétois, Positive clusters for smooth perturbations of a critical elliptic equation in dimensions four and five, J. Funct. Anal. (2016), Preprint, 24 pages.

30. Neil S. Trudinger, Remarks concerning the conformal deformation of Riemannian structures on compact manifolds, Ann. Scuola Norm. Sup. Pisa (3) 22 (1968), 265-274. MR 0240748

31. Jérôme Vétois and Shaodong Wang, Infinitely many solutions for cubic nonlinear Schrödinger equations in dimension four, Advances in Nonlinear Analysis (2017), To appear.

32. Juncheng Wei, Existence and stability of spikes for the Gierer-Meinhardt system, Handbook of differential equations: stationary partial differential equations. Vol. V, Handb. Differ. Equ., Elsevier/NorthHolland, Amsterdam, 2008, pp. 487-585. MR 2497911 (2011b:35214)

Bruno Premoselli, Université Libre de Bruxelles, Service de Géométrie Différentielle

CP 218, Boulevard du Triomphe, B-1050 Bruxelles, Belgique.

E-mail address: bruno.premoselli@ulb.ac.be,

Pierre-Damien Thizy, Laboratoire AGM, Université de Cergy-Pontoise site Saint-Martin, 2 avenue Adolphe Chauvin 95302 Cergy-Pontoise cedex France.

E-mail address: pierre-damien.thizy@u-cergy.fr, 